\title{
On Quantum Gauge Theories in Two Dimensions
}

\author{
Edward Witten * \\ School of Natural Sciences, Institute for Advanced Study, Olden Lane, Princeton, NJ 08540, USA
}

Received February 22, 1991

\begin{abstract}
Two dimensional quantum Yang-Mills theory is studied from three points of view: (i) by standard physical methods; (ii) by relating it to the large $k$ limit of three dimensional Chern-Simons theory and two dimensional conformal field theory; (iii) by relating its weak coupling limit to the theory of ReidemeisterRay-Singer torsion. The results obtained from the three points of view agree and give formulas for the volumes of the moduli spaces of representations of fundamental groups of two dimensional surfaces.
\end{abstract}

\section{Introduction}

Let $\Sigma$ be a compact two dimensional manifold. Pick a compact gauge group $G$. Let $E$ be a $G$ bundle over $\Sigma$, with a connection $A$ and curvature $F=d A=A \wedge A$. $F$ is a two form with values in the adjoint bundle, which we will denote as $\operatorname{ad}(E)$.

Let $\mathscr{M}$ be the moduli space of flat connections on $E$, up to gauge transformation. If $\Sigma$ is orientable, $\mathscr{M}$ has a natural symplectic structure $\omega[1,2]$ and thus a natural volume form

$$
\theta=\frac{\omega^{n}}{n !}
$$

where $n=\operatorname{dim} \mathscr{M} / 2$. Even if $\Sigma$ is not orientable, a natural measure on $\mathscr{M}$ can be defined using the theory of Reidemeister torsion (or Ray-Singer analytic torsion [3]); this measure agrees with $\theta$ in the orientable case, as we will verify in Sect. 4.2. The volume $\operatorname{Vol}(\mathscr{M})$ of $\mathscr{M}$, obtained by integrating this measure, will be our main interest in this paper.

If $\Sigma$ is orientable, this volume is a rational number for the following reason. The symplectic structure $\omega$ has integral periods and represents the first Chern class of a certain natural line bundle over $\mathscr{M}$. Hence $\theta$ represents a rational class (the

* Research supported in part by NSF Grant PHY86-20266 
denominator coming from the explicit $n$ ! in (1.1)). No such rationality of $\operatorname{Vol}(\mathscr{M})$ can be expected in the unorientable case.

In the orientable case, a formula for $\operatorname{Vol}(\mathscr{M})$ can be obtained by picking a complex structure $J$ on $\Sigma$, so that $\mathscr{M}$ can be identified (by a theorem of Narasimhan and Seshadri [4]) with the moduli space $\mathscr{M}_{J}$ of stable holomorphic $G_{\mathbb{C}}$ bundles on $\Sigma$ (of topological type determined by $E$ ). Moreover, the line bundle $\mathscr{L}$ can then be identified as a holomorphic line bundle over $\mathscr{M}_{\boldsymbol{J}}$. Verlinde [5] extracted from physical arguments in conformal field theory a formula, which we will recall in Sect. 3, for the dimension of $H^{0}\left(\mathscr{M}_{J}, \mathscr{L}^{\otimes k}\right)$. (A rigorous proof of the Verlinde formula as a statement about the number of conformal blocks in conformal field theory can be found in [6], and the relation of this with $H^{0}\left(\mathscr{M}_{J}, \mathscr{L}^{\otimes k}\right)$ is sketched in [7], along with a sketch of a short proof of the Verlinde formula. A direct algebrageometric proof of Verlinde's formula has not yet been given.) By the RiemannRoch formula, the volume of $\mathscr{M}$ is

$$
\operatorname{Vol}(\mathscr{M})=\lim _{k \rightarrow \infty} k^{-n} \operatorname{dim} H^{0}\left(\mathscr{M}_{J}, \mathscr{L}^{\otimes k}\right),
$$

and so can be extracted from Verlinde's formula. For instance, as we will see in Sect. 3, for $G=S U(2)$, and $\Sigma$ a Riemann surface of genus $g$, one finds $\operatorname{Vol}(\mathscr{M})$ $=2 \cdot\left(2 \pi^{2}\right)^{1-g} \cdot \zeta(2 g-2)$, where $\zeta(s)$ is the Riemann zeta function. Rationality of this number follows from Euler's well known evaluation of the zeta function at positive even integers [9]. Independently of the present work, Thaddeus [10] has recently extracted this result and many other topological consequences of Verlinde's formula.

As we will see in Sect. 3, in connection with Eq. (3.18) for the volume of twisted moduli spaces, the rationality of the Hurwitz zeta function (closely related to Dirichlet $L$-functions) at special values can also be seen as a consequence of Verlinde's formula.

Verlinde's formula is based on fairly sophisticated physics or mathematics. The main purpose of this paper is to present a computation of $\operatorname{Vol}(\mathscr{M})$ based on much more elementary arguments. This computation does not contain all the information present in Verlinde's formula, but at least in the semiclassical limit of large $k$, it renders transparent the most mysterious part of Verlinde's results, which is the "diagonalization of the fusion rules."

This "elementary" computation of $\operatorname{Vol}(\mathscr{M})$ also has the virtue that it extends to the case that $\Sigma$ is unorientable. For instance, for $G=S U(2)$ and $\Sigma$ the connected sum of an orientable surface of genus $g$ with the projective plane $R P^{2}$, the volume of $\mathscr{M}$ is an elementary multiple of $\zeta(2 g-1)$ (as we will see in Sect. 4.6). In contrast to the values of the zeta function at positive even integers, the values of $\zeta(s)$ at positive odd integers are little understood; there is no reason to expect the multiple of $\zeta(2 g-1)$ that arises here to be rational. Of course, we do not expect any rationality when $\Sigma$ is unorientable.

Certain generalizations are also natural. One involves deleting $n$ points $P_{1}, \ldots, P_{n}$ from $\Sigma$ and considering the moduli space of flat connections on $\Sigma-\left\{P_{1}, \ldots, P_{n}\right\}$ with prescribed monodromies around the $P_{i}$. We will compute the volumes of these more general moduli spaces. Another generalization (which turns out to be related) involves computing intersection numbers of certain algebraic cycles on $\mathscr{M}$. It will not be pursued here.

This paper is organized as follows. In Sect. 2, we introduce the quantum gauge theories on which the analysis will focus, and describe a physical version of the computation of $\operatorname{Vol}(\mathscr{M})$ and some of its generalizations. In Sect. 3, we review the 
Verlinde formulas. In Sect. 4, we carry out a mathematically precise version of the calculation of $\operatorname{Vol}(\mathscr{M})$, using the interpretation as Reidemeister torsion. This computation proceeds by imitating the steps in Sect. 2. The background material to Sect. 4 (which we have, however, tried to make self-contained) can be found in [11-13]. This paper is written in such a way that it can be understood while omitting certain parts of Sect. 2 or most of Sect. 4.

Two dimensional quantum Yang-Mills theory, which is our basic tool in Sect. 2, has previously been analyzed by Fine [14] using a fairly explicit analysis of the continuum path integral. His results agree with ours at least in genus zero (where he obtains explicit formulas). I understand that results similar to part of Sect. 2 were also obtained some years ago in unpublished work by W. Nahm and K. Uhlenbeck.

\section{The Quantum Theories}

We will be studying certain quantum gauge theories, with compact gauge group $G$, on a two dimensional manifold $\Sigma$. To define these theories, we must pick an invariant quadratic form on the Lie algebra $\mathscr{G}$. If $G$ is simple (an assumption whose role is only to simplify the notation in what follows), this quadratic form is unique up to normalization. Though the normalization will not be important in this section, we will find a certain choice useful in Sect. 3. The Lie algebra $\mathscr{G}$ of $G$ coincides with that of the universal cover $G^{\prime}$ of $G$. A quadratic form (, ) on $\mathscr{G}$ determines a characteristic class $\alpha \in H^{4}\left(B G^{\prime}, \mathbb{R}\right)$, which can be represented in de Rham theory by the four form

$$
\frac{1}{8 \pi^{2}}(F, F),
$$

where $F$ is the curvature of a connection on the universal bundle over $B G^{\prime}$. We pick (, ) so that (2.1) is the fundamental integer-valued characteristic class of $G^{\prime}$ bundles (i.e., $\alpha$ generates the image of $H^{4}\left(B G^{\prime}, \mathbb{Z}\right)$ in $H^{4}\left(B G^{\prime}, \mathbb{R}\right)$ ). For $G=S U(N)$, one has $(a, b)=-\operatorname{Tr} a b$, where $\operatorname{Tr}$ is the trace in the $N$ dimensional representation. Motivated by this example, we denote (, ) as $\operatorname{Tr}$ for any $G^{1}$.

The Yang-Mills action or Lagrangian on a Riemannian manifold $\Sigma$ of any dimension $n$ is the functional of connections defined by

$$
I(A)=-\frac{1}{4 e^{2}} \int_{\Sigma} d \mu g^{i k} g^{j l} \operatorname{Tr} F_{i j} F_{k l},
$$

where $g_{i j}$ is the metric of $\Sigma, \mu$ is the Riemannian measure, and $e$ is a real constant, the gauge coupling constant. It has long been known [15] that for $\Sigma$ of dimension two, the quantum field theory associated with this action is exactly soluble and indeed trivial locally. At a fundamental level, the reason for this is that in two dimensions, the Yang-Mills action depends only on the measure $\mu$ determined by the metric $g$. In particular, (2.2) is invariant under the group of area preserving diffeomorphisms of $\Sigma$. This is such a large group that the quantum theory associated with (2.2), which we might characterize as an area preserving quantum field theory, has a flavor similar to topological field theory.

\footnotetext{
1 We consider the Lie algebra of a compact group to consist of anti-hermitian matrices, so the quadratic form $(a, b)=-\operatorname{Tr} a b$ is positive definite
} 
To exhibit the fact that in two dimensions, (2.2) really only depends on the choice of a measure, note that if $\Sigma$ is oriented, a metric determines a volume form $\varepsilon$, and we can define an ad $(E)$-valued zero form $f$ by $F=\varepsilon f$. For $\Sigma$ unorientable, $\varepsilon$ does not exist as a two form, but still exists as a two form with values in the orientation bundle $\sigma$ (which is a real line bundle with structure group $\mathbb{Z}_{2}$ ). In that case we can still write $F=\varepsilon f$ with $f$ an ad $(E)$-valued section of $\sigma$. The action can then be written

$$
I(A)=-\frac{1}{2 e^{2}} \int_{\Sigma} d \mu \operatorname{Tr} f^{2}
$$

making explicit the fact that it depends only on a choice of measure, and not on a metric.

The only invariant of a measure on a Riemann surface $\Sigma$ is the total area,

$$
\varrho=\int_{\Sigma} d \mu .
$$

Note that $I(A)$ is invariant under $e^{2} \rightarrow t e^{2}, \mu \rightarrow \mu / t$ for any real number $t$. (The relation $F=\varepsilon f$ shows that under such a scaling, one has $f \rightarrow t f$.)

The quantity of prime interest in this paper will be the partition function

$$
Z_{\Sigma}\left(e^{2}, \varrho\right)=\int D A e^{-I(A)} .
$$

Here $D A$ denotes the Feynman path integral over the space of connections (divided by the volume of the gauge group, as we will discuss later). In the simple two dimensional theory under consideration here, this integral can be defined rigorously by a lattice regularization [15] that we will recall in Sect. 2.3. Because of the scaling law noted in the last paragraph, $Z_{\Sigma}$ in fact is a function only of the invariant combination $e^{2} \varrho$, so we will denote it as $Z_{\Sigma}\left(e^{2} \varrho\right)$ or simply as $Z\left(e^{2} \varrho\right)$ if $\Sigma$ is understood.

Comparison of Different Regularizations. We will now make a technical discursion. The quantum field theory just introduced is superrenormalizable according to the standard criteria, and in perturbation theory one encounters no infinities involving the gauge fields. However, the one loop amplitude in an external gravitational field has quadratic and logarithmic divergences that (in an arbitrary regularization scheme) can be renormalized by adding to the Lagrangian multiples of $\int_{\Sigma} d \mu$ and $\int_{\Sigma} d \mu R$, respectively. ( $R$ is the curvature scalar.) Thus, we should more properly begin with a Lagrangian containing counterterms depending on the metric and topology of $\Sigma$ :

$$
I(A, g)=I(A)+u \int_{\Sigma} d \mu+v \int_{\Sigma} d \mu \frac{R}{2 \pi} .
$$

(The two counterterms are of course the volume and Euler characteristic of $\Sigma$.) As is standard in such superrenormalizable theories, the partition functions $Z_{\Sigma}\left(e^{2} \varrho\right)$ and $Z_{\Sigma}^{\prime}\left(e^{2} \varrho\right)$ computed in two different regularizations will differ by terms that can be interpreted as corrections to $u$ and $v$ :

$$
Z_{\Sigma}^{\prime}\left(e^{2} \varrho\right)=Z_{\Sigma}\left(e^{2} \varrho\right) \exp \left(\Delta u \int_{\Sigma} d \mu+\Delta v \int_{\Sigma} d \mu \frac{R}{2 \pi}\right),
$$


with $\Delta u$ and $\Delta v$ being constants (independent of $\Sigma$ but perhaps depending on $e$ ). (In other words, what is regarded in one regularization as the partition function at $u=v=0$ is interpreted in the other regularization as the partition function at some nonzero values of $u$ and $v$.) If the scaling symmetry under $e^{2} \rightarrow t e^{2}, \varrho \rightarrow \varrho / t$ is maintained in each regularization (as we will ensure), then

$$
\Delta u=e^{2} u_{0}, \quad \Delta v=v_{0},
$$

with $u_{0}, v_{0}$ being independent of $e^{2}$.

In practice, we will make careful comparisons of different regularizations only in the limit $e \rightarrow 0$ (and similar topologically invariant situations). Equation (2.8) shows that the ambiguity in the definition of $u$ will not affect such comparisons. On the other hand, the ambiguity in the definition of $v$ corresponds to a one parameter ambiguity

$$
Z_{\Sigma}(0) \rightarrow e^{\Delta v \cdot \chi(\Sigma)} \cdot Z_{\Sigma}(0)
$$

in the definition of the partition function in the limit $e=0$. Such factors will appear in this paper when we compare calculations based on a lattice regularization to calculations of a more precise type.

\subsection{Some Related Theories}

Much of the interest of the two dimensional Yang-Mills theory that we have introduced above derives from certain alternative interpretations that it possesses. First of all, let $\phi$ be an $\operatorname{ad}(E)$ valued zero form (or in the unorientable case, an ad(E) valued section of $\sigma$ ), and consider the action functional

$$
I^{\prime}(A, \phi)=-\frac{e^{2}}{2} \int_{\Sigma} d \mu \operatorname{Tr} \phi^{2}-i \int_{\Sigma} \operatorname{Tr} \phi F,
$$

for a theory in which the dynamical variables are $\phi$ and $A$. The partition function is

$$
\tilde{Z}_{\Sigma}\left(e^{2} \varrho\right)=\int D \phi D A e^{\frac{e^{2}}{2} \int d \mu \operatorname{Tr} \phi^{2}} e^{i \int \operatorname{Tr} \phi F} .
$$

Performing first the Gaussian integral over $\phi$, using

$$
\int_{-\infty}^{+\infty} \frac{d x}{\sqrt{2 \pi}} e^{-\frac{e^{2}}{2} x^{2}-i x y}=e^{-y^{2} / 2 e^{2}},
$$

we find that (2.11) reduces to (2.5), so

$$
\tilde{Z}_{\Sigma}\left(e^{2} \varrho\right)=Z_{\Sigma}\left(e^{2} \varrho\right) .
$$

The advantage of this formulation is that it is relatively straightforward to take the limit $e \rightarrow 0$ of (2.10). In fact at $e=0$, the action is

$$
I^{\prime \prime}(A, \phi)=-i \int_{\Sigma} \operatorname{Tr} \phi F,
$$

and the partition function is

$$
Z_{\Sigma}(0)=\int D \phi D A e^{i \int \operatorname{Tr} \phi F} .
$$

This theory is topologically invariant; the measure $d \mu$ no longer enters. Indeed (2.15) is a slightly nonlinear variant of the quantum field theory that was 
introduced many years ago by Schwarz [16] in connection with Ray-Singer analytic torsion. We will explain how the analytic torsion enters in Sect. 2.2.

If one's interest is to study the partition function $Z_{\Sigma}(0)$ of the topological field theory (2.14), one might think that one should tackle this directly instead of the generalization $Z_{\Sigma}\left(e^{2} \varrho\right)$. However, we will see that considering the generalization makes obvious a certain "diagonalization" (which is related to Verlinde's diagonalization of the fusion rules) which drastically simplifies the computation.

To complete the story, there is one more closely related theory that should be considered. This is the three dimensional Chern-Simons theory. Let $B$ be a connection on a $G$ bundle $E$ on an oriented three manifold $X$. Suppose for simplicity that $E$ is trivial. Picking a trivialization, identify $B$ as an $\operatorname{ad}(E)$ valued one form. The Chern-Simons action is then

$$
I_{\text {C.S. }}(B)=-\frac{i k}{4 \pi} \int_{X} \operatorname{Tr}\left(B \wedge d B+\frac{2}{3} B \wedge B \wedge B\right),
$$

where $k$ is a positive integer, the level. The Feynman path integral is

$$
Z_{X}(k)=\int D B^{\prime} \exp \left[\frac{i k}{4 \pi} \int_{X} \operatorname{Tr}\left(B \wedge d B+\frac{2}{3} B \wedge B \wedge B\right)\right] .
$$

Now take $X=\mathbf{S}^{1} \times \Sigma$, where $\mathbf{S}^{1}$ is the circle $0 \leqq t \leqq 1$ (with $t=0$ and $t=1$ identified), and $\Sigma$ is an oriented surface. Let $w: \mathbf{S}^{1} \times \overline{\bar{\Sigma}} \rightarrow \Sigma$ be the projection. For connections of the special form

$$
B=w^{*}(\phi) d t+w^{*}(A),
$$

where $\phi$ and $A$ are $\operatorname{ad}(E)$ valued zero and one forms on $\Sigma$, the Chern-Simons action is

$$
I_{\text {C.S. }}(B)=-\frac{i k}{2 \pi} \int_{\Sigma} \operatorname{Tr} \phi F(A),
$$

and so coincides with the topological action (2.14), apart from a factor of $k / 2 \pi$. This factor can be absorbed in the definition of $\phi$ at the cost of multiplying the partition function by a factor of the type (2.9), for some value of $\Delta v$. A factor of this type will appear because two theories (such as (2.14) and (2.19)) that are equivalent classically will differ quantum mechanically only by a renormalization effect. For the topologically invariant theories (2.14) and (2.19), the $\Delta v$ term is the only relevant renormalization.

In general, we are not entitled in quantum theory to arbitrarily restrict to connections of the form (2.18); we wish to evaluate the Feynman integral (2.17) over all connections. However, for large $k$, the integral for $Z_{X}(k)$ is dominated by stationary phase, expanding around flat connections. If the center of $G$ is trivial, an irreducible flat connection on $X=\mathbf{S}^{1} \times \Sigma$ is the pullback of a flat connection on $\Sigma^{2}$. More generally, if the center of $G$ is a finite set with \#Z(G) elements, then the moduli space $\mathscr{M}_{X}$ of irreducible flat connections on $X$ consists of \# $Z(G)$ copies of the moduli space $\mathscr{M}$ of irreducible flat connections on $\Sigma$; the factor of $\# Z(G)$ arises because, given an irreducible flat connection in the $\Sigma$ directions, one can form a flat connection on $X$ by taking the holonomy around $\mathbf{S}^{1}$ to be any element of the

\footnotetext{
${ }^{2}$ The reducible flat connections can be seen to make subdominant contributions for large $k$
} 
center of $G$. In the semi-classical evaluation of the Chern-Simons partition function for large $k$, each of the $\# Z(G)$ copies of $\mathscr{M}$ make the same contribution. The Chern-Simons partition function for large $k$ is hence $\# Z(G)$ times the partition function of the two dimensional theory (2.19). As (2.19) differs from (2.14) only by a rescaling of $\phi$, which brings the renormalization effect indicated at the end of the last paragraph, the relation between the large $k$ behavior of the ChernSimons partition $Z_{X}(k)$ and the partition function $Z_{\Sigma}(0)$ of $(2.14)$ is

$$
Z_{X}(k) \sim \# Z(G) \cdot e^{\Delta v \cdot x(\Sigma)} \cdot Z_{\Sigma}(0),
$$

where $\Delta v$ may depend on $k$ but not on $\Sigma$. With an explicit choice (such as zeta function regularization) for defining the determinants that appear in evaluating the left- and right-hand sides of (2.20), an a priori computation of $\Delta v$ can be given. We will not do so in this paper.

\subsection{Extracting the Torsion}

We now wish to explain the relation of the partition function (2.15) with Reidemeister-Ray-Singer torsion.

First we briefly recall the Fadde'ev-Popov-BRST gauge fixing of the gauge theory with Lagrangian (2.14). Let $\hat{G}$ be the group of maps of $\Sigma$ to $G$. The path integral over the space of all connections is divergent because of gauge invariances, and one wishes, formally, to render it finite by dividing by the volume of $\hat{G}$. Formally one would like to integrate over the space $\mathscr{A}$ of all connections and then divide by the volume of $\hat{G}$. These two operations are each ill-defined, and one considers instead the better-defined procedure of integrating over the quotient $\mathscr{A} / \hat{G}$. There is a subtlety (usually neglected) that we have to take into account here. Let $D A$ denote the formal path integral measure over $\mathscr{A}$, let $\pi: \mathscr{A} \rightarrow \mathscr{A} / \hat{G}$ be the projection, and let $\hat{\mu}$ be Haar measure on $\hat{G}$. Formally, the quotient measure $D A^{\prime}$ on $\mathscr{A} / \hat{G}$ is the measure such that $D A=\pi^{*}\left(D A^{\prime}\right) \cdot \hat{\mu}$. In a finite dimensional analog of this situation (such as we will frequently encounter in Sect. 4), one has

$$
\frac{1}{\operatorname{vol} \widehat{G}} \int D A e^{-\mathscr{L}}=\int D A^{\prime} e^{-\mathscr{L}}
$$

provided that $\hat{G}$ acts freely on $\mathscr{A}$, at least generically. In the present case, this is true only if the center of the gauge group $G$ is trivial. Indeed, the gauge transformations that leave invariant a generic connection are precisely the constant maps of $\Sigma$ to the center of $G$. Let $\# Z(G)$ denote the number of elements in that center. The general formula that replaces $(2.21)$ is

$$
\frac{1}{\operatorname{vol} \hat{G}} \int D A e^{-\mathscr{L}}=\frac{1}{\# Z(G)} \int D A^{\prime} e^{-\mathscr{L}} .
$$

The Fadde'ev-Popov procedure constructs $\int D A^{\prime} e^{-\mathscr{L}}$, and as what we want is the left-hand side of (2.22), we will have to divide by hand by a factor of \#Z(G). [In most physical applications, this factor can be neglected since it cancels out when one computes correlation functions. We have assumed $G$ semi-simple, so that $\# Z(G)$ is finite. In general, this factor would be replaced by the volume of the center of $G$, computed with an appropriate measure.] 
The Fadde'ev-Popov-BRST procedure for constructing the quotient measure on $\mathscr{A} / \hat{G}$ involves introducing the standard ghost field $c$, which is an anticommuting zero form with values in $\operatorname{ad}(E)$, and BRST transformation laws

$$
\begin{aligned}
\delta A_{i} & =-D_{i} c=-\left(\partial_{i} c+\left[A_{i}, c\right]\right), \\
\delta c & =\frac{1}{2}[c, c] .
\end{aligned}
$$

One also requires an antighost multiplet. This is conveniently chosen to consist of an anticommuting zero form $\bar{c}$ and a commuting zero form $w$ with values in $\operatorname{ad}(E)$, with

$$
\delta \bar{c}=i w, \quad \delta w=0 .
$$

The gauge fixing term is $I_{\mathrm{GF}}=-\delta V$ with a suitable $V$. Even though no metric appears in the classical action (2.14), natural choices of $V$ depend on a choice of metric on $\Sigma$.

Actually, there is a topological obstruction (coming from the topology of $\hat{G}$ ) to finding any suitable global choice of $V$. We make appropriate local choices, each suitable for computing the path integral over a certain region in the space of fields modulo gauge transformations, and patch together the results (after verifying independence of the local choices). In a neighborhood of any connection $A_{(0)}$, writing $A=A_{(0)}+B$, and letting $D_{i}^{(0)}$ be the covariant derivative at the connection $A_{(0)}$, a convenient local choice is

$$
V=-\int_{\Sigma} d \mu \operatorname{Tr} \bar{c} D_{i}^{(0)} B^{i} .
$$

This gives

$$
I_{\mathrm{GF}}=\int_{\Sigma} d \mu \operatorname{Tr}\left(+i w D_{i}^{(0)} B^{i}-\bar{c} D_{i}^{(0)} D^{i} c\right) .
$$

The Euler-Lagrange equation obtained by varying this action with respect to $w$ is

$$
0=D_{i}^{(0)} B^{i},
$$

and this is the gauge condition placed on the quantum theory if it is gauge fixed with this choice of $V$.

Allowing for the factor explained in (2.22), the partition function (2.15) is now

$$
\begin{aligned}
Z_{\Sigma}(0)= & \frac{1}{\# Z(G)} \int D A D \phi D c D \bar{c} D w \\
& \times \exp \left(i \int_{\Sigma} \operatorname{Tr} \phi F-i \int_{\Sigma} d \mu w D_{i}^{(0)} B^{i}-\int_{\Sigma} d \mu \operatorname{Tr} D_{i}^{(0)} \bar{c} D^{i} c\right) .
\end{aligned}
$$

The Orientable Case. We will now try to clarify the origin of the integration measure. In doing so, we first assume that $\Sigma$ is orientable. The space $\mathscr{A}$ of connections on $\Sigma$ has in that case a natural symplectic structure. Indeed, a tangent vector to $\mathscr{A}\left(\right.$ at some base connection $\left.A_{(0)}\right)$ is an $\operatorname{ad}(E)$-valued one form. Given two such one forms $\alpha$ and $\beta$, one defines the skew pairing

$$
\langle\alpha, \beta\rangle=\frac{1}{4 \pi^{2}} \int_{\Sigma} \operatorname{Tr} \alpha \wedge \beta,
$$

which defines a symplectic structure on $\mathscr{A}$. Formally, $\mathscr{A}$, as a symplectic manifold, acquires a measure. 
By contrast, the space $\mathscr{E}$ of $\operatorname{ad}(E)$ valued zero forms does not have a natural measure. Any measure on $\Sigma$ (such as the Riemannian measure of the metric that was used in the definition of $V$ ) determines a metric on $\operatorname{ad}(E)$, say

$$
(f, g)=-\frac{1}{4 \pi^{2}} \int_{\Sigma} d \mu \operatorname{Tr}(f g) \text {. }
$$

A metric formally determines a Riemannian measure on $\operatorname{ad}(E)$ (namely $\prod_{i} d f_{i}$, where the $f_{i}$ are an orthonormal basis of $\mathscr{E}$ ). Given a metric on $\Sigma$ (actually a conformal structure is enough), we can also define a metric on $\mathscr{A}$ :

$$
(\alpha, \beta)=\frac{1}{4 \pi^{2}} \int_{\Sigma} \operatorname{Tr} \alpha \wedge * \beta .
$$

The metric (2.31) and symplectic structure (2.29) are compatible in the sense that together they define a Kahler structure.

In (2.28), $c, \bar{c}, \phi$, and $w$ are all zero forms with values in $\operatorname{ad}(E)$, but $\phi$ and $w$ are bosonic while $c, \bar{c}$ are fermionic. Though there is no natural measure separately on the space of bosonic or fermionic sections of $\operatorname{ad}(E)$, the ambiguity cancels formally when one has equal numbers of bosons and fermions. (Under a change of metric in (2.30), the Riemannian measure on $\mathscr{E}$ changes by a Jacobian factor which appears in the numerator for bosons, and in the denominator for fermions.) In practice, therefore, we can consider the measure in the integration over $c, \bar{c}, \phi$, and $w$ to be determined by any metric on ad $(E)$, as long as we treat all these fields alike. We will ensure this by using the zeta function definition of determinants [3].

To evaluate (2.28), we perform the integral first over $\phi$ and $w$. Using again (2.12), we get

$$
Z_{\Sigma}(0)=\frac{1}{\# Z(G)} \cdot \int D A D c D \bar{c} \exp \left(-\int_{\Sigma} d \mu \operatorname{Tr} D_{i}^{(0)} \bar{c} D^{i} c\right) \cdot \prod_{x \in \Sigma} \delta(F(x)) \delta\left(D^{(0)} * B(x)\right) .
$$

The equations

$$
0=F=D^{(0)} * B
$$

are local equations which cut out the moduli space $\mathscr{M}$ of flat connections, inside the space $\mathscr{A}$ of all connections. At this point we may as well suppose that the base connection $A_{(0)}$ is flat, so that $B=0$ is a solution of (2.33). Temporarily let us suppose (unrealistically) that it is an isolated solution. Equation (2.33) is a nonlinear equation for $B$ (as $F$ has a quadratic term). The linearization of this equation is an elliptic equation $Q B=0$, where $Q: \Omega^{1}(\operatorname{ad}(E))$ $\rightarrow \Omega^{2}(\operatorname{ad}(E)) \oplus \Omega^{0}(\operatorname{ad}(E))$ is the operator

$$
Q=D \oplus * D * .
$$

The general principle in performing the integral over $A$ in (2.32) is that if $H: R^{n} \rightarrow R^{n}$ is a smooth map with an isolated nondegenerate zero at the origin, and $H^{\prime}$ is the linear operator obtained by linearizing $H$ near $x_{i}=0$, then

$$
\int d x_{1} \ldots d x_{n} \delta^{n}(H(x))=\frac{1}{\left|\operatorname{det} H^{\prime}\right|} .
$$


On the other hand, the integral over $c$ and $\bar{c}$ in (2.32) is Gaussian, and gives the determinant of the corresponding elliptic operator, which is the Laplacian $\Delta_{0}=* D * D$ (acting on zero forms; $\Delta_{q}$ will denote the Laplacian $* D * D+D * D *$ acting on $q$ forms). Hence, if $B=0$ were an isolated solution of (2.33), the result of the path integral would be

$$
\frac{1}{\# Z(G)} \cdot \frac{\operatorname{det} \Delta_{0}}{|\operatorname{det} Q|} .
$$

The particular combination of determinants of elliptic operators appearing in (2.36) is the analytic torsion of the flat connection $A_{(0)}[3]$. The role of analytic torsion in quantum field theory was first explained by Schwarz [16]. A computation just like the present one also arises (for the same reason) in three dimensional gravity with zero cosmological constant [17].

One can readily show that $|\operatorname{det} Q|=\sqrt{\operatorname{det} \Delta_{0} \operatorname{det} \Delta_{2}} \cdot{ }^{3}$ Hence, the contribution of an isolated flat connection $A_{(0)}$ would be $\sqrt{\operatorname{det} \Delta_{0} / \operatorname{det} \Delta_{2}} \cdot(\# Z(G))^{-1}$. It is also true that on an orientable manifold, $\Delta_{0}$ and $\Delta_{2}$ have the same spectrum, and thus the same determinants (as the * operator gives a natural identification between them). So actually, on an orientable manifold, an isolated irreducible flat connection would just contribute $1 / \# Z(G)$. This is a special case of the statement that "on an even dimensional orientable manifold, the analytic torsion is trivial."

Now we must consider the more realistic case in which the solution $B=0$ to (2.33) is not isolated. (We will, however, assume that $\Delta_{0}$ has no kernel, and this means that we are restricted to genus $\geqq 2$.) In this case, the solutions to (2.33) are a vector space which can be identified with $T \mathscr{M}$, the tangent space to the moduli space of flat connections. We write

$$
\Omega^{1}(\operatorname{ad}(E))=T \mathscr{M} \oplus \Omega_{\perp}^{1},
$$

where $\Omega_{\perp}^{1}$ is the orthocomplement to $T \mathscr{M}$. The symplectic form (2.29) restricts to symplectic forms, and hence measures, on both $T \mathscr{M}$ and $\Omega_{\perp}^{1}$. The induced symplectic form on $T \mathscr{M}$ is the one associated with the natural symplectic form $\omega$ on $\mathscr{M}$, which enters in the formula (1.1) for the natural volume form on $\mathscr{M}$. A priori, the measure on $\mathscr{M}$ defined by the path integral is not simply this elementary, natural measure, but receives a correction factor from the rest of the path integral. Repeating the derivation of (2.36), the correction factor is

$$
w=\frac{1}{\# Z(G)} \frac{\operatorname{det} \Delta_{0}}{\left|\operatorname{det} Q_{\perp}\right|},
$$

where now $Q_{\perp}=\left.Q\right|_{\Omega^{1}{ }_{\perp}}$. By the facts cited in the last paragraph, this correction factor is just $1 / \# Z(G)$ for $\Sigma$ orientable.

Thus, the "triviality" of torsion in this situation means simply that the measure on $\mathscr{M}$ determined by the torsion - or by the quantum field theory (2.14) - reduces to the elementary measure defined by the symplectic structure, divided by the elementary factor \# $Z(G)$. The reason that this fact is useful is that the torsion - or the quantum field theory - can be studied by powerful methods, which are not directly available for the symplectic volume, despite its elementary definition.

${ }^{3}$ First, $|\operatorname{det} Q|=\sqrt{\operatorname{det} Q Q^{*}}$. (Here $Q^{*}$ is the operator $D \oplus * D *: \Omega^{0} \oplus \Omega^{2} \rightarrow \Omega^{1}$.) But $Q Q^{*}=\Delta_{0} \oplus \Delta_{2}$, so $|\operatorname{det} Q|=\sqrt{\operatorname{det} Q Q^{*}}=\sqrt{\operatorname{det} \Delta_{0} \cdot \operatorname{det} \Delta_{2}}$ 
The Unorientable Case. Let us now consider the case of $\Sigma$ unorientable. One then does not have a symplectic structure (2.29) on the space of connections. It is therefore less obvious why the quantum field theory associated with the Lagrangian (2.14) works. I will not attempt a full explanation, but just state some facts. Though there is no symplectic structure on $\mathscr{A}$, once a metric is picked on $\Sigma$, a metric on $\mathscr{A}$ can be defined by the formula (2.31). This induces a metric, and hence a measure, on the fiber of $T \mathscr{M}$ at an arbitrary point $A_{(0)}$; hence, it induces a measure $\tau_{0}$ on $\mathscr{M}$. This measure is not "natural" but depends on the conformal structure used in (2.31). The correction factor $w$ from the path integral is still given by (2.38) and is not simply $1 / \# Z(G)$ in the unorientable case. The measure on $\mathscr{M}$ determined by the quantum field theory (2.14) is $\tau=w \tau_{0}$. The proof of topological invariance of analytic torsion [3] means that $\tau$ is independent of the metric on $\Sigma$. A quantum field theorist would formulate this argument as an expression of the fact that the dependence of the gauge fixed Lagrangian [the exponent in (2.28)] on the metric on $\Sigma$ is a BRST commutator. The tools involved in formulating the argument this way are similar to those that enter in establishing the topological invariance of three dimensional quantum Chern-Simons theory. (See e.g. the last section of [30].)

\subsection{Combinatorial Treatment}

We will now explain how a combinatorial approach (originally introduced by Migdal [15] for the case $\Sigma=\mathbb{R}^{2}$ ) can be used to compute the partition function $Z_{\Sigma}\left(e^{2} \varrho\right)$. We may as well set $e^{2}=1$.

In general, to make sense of the Feynman path integral over some function space $\mathscr{W}$, it is sometimes convenient to make an approximation to $\mathscr{W}$ of some finite dimension $n$. One then tries to prove that the limit as $n \rightarrow \infty$ exists and is independent of whatever arbitrary choices may have been made in constructing the finite dimensional approximation. In Yang-Mills theory, a finite dimensional approximation can be made via "lattice gauge theory" [18]. One "triangulates" $\Sigma$, or more generally one covers it by polygons, not necessarily triangles (Fig. 1). One restricts the bundle $E$ to the finite set $\mathscr{S}$ consisting of the vertices of the polygons. (Of course, this is a drastic step in which the topology of $E$ is lost, and the subsequent computation therefore gives the Yang-Mills partition function summed over all topological types of $G$ bundle on $\Sigma$.) In lattice gauge theory, the interiors of the polygons are usually called plaquettes. A lattice gauge transformation is a map from $\mathscr{S}$ to $G$, that is, an assignment of a group element $g_{x} \in G$ to every $x \in \mathscr{S}$. One then introduces a lattice version of a connection in which one considers parallel transport only along the edges of the polygons. To every edge $\gamma$, with endpoints $x$ and $y$, one assigns a $G$ element $U_{y}$, regarded as the operator of parallel transport from $x$ to $y$. $U_{y}$ should be thought of as a map $E_{x} \rightarrow E_{y}$, and under a gauge transformation it transforms as

$$
U_{\gamma} \rightarrow g_{y} U_{\gamma} g_{x}^{-1} .
$$

One then tries to define a suitable lattice version of the Yang-Mills action. In any dimension, it is possible to approximate Yang-Mills theory in this way, and in dimensions $\leqq 4$ it is believed (and in some instances proved) that in the limit as the triangulation becomes finer, the desired continuum theory can be recovered. What is special about dimension two is that it is possible [15] to formulate the lattice theory in such a way that the partition function is invariant under subdivision of the lattice. This is what we now wish to explain. 


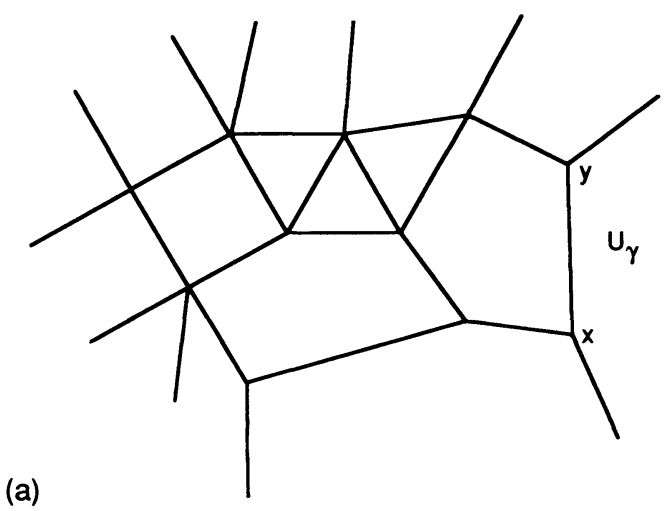

(b)
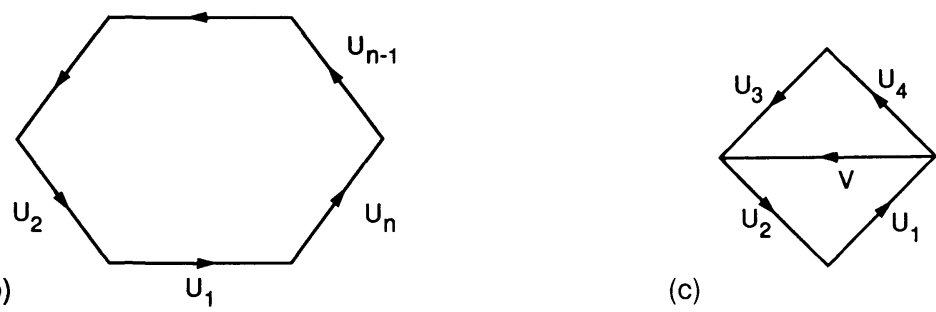

(c)

Fig. 1a-c. Covering a surface $\Sigma$ by polygons (a). $U_{y}$ is an edge with endpoints $x$ and $y$. A plaquette (b) with connection elements $U_{1}, \ldots, U_{n}$ associated with the edges and holonomy $\mathscr{U}=U_{1}, \ldots, U_{n}$. Subdivision of one of the plaquettes (c)

The integrand in the Feynman path integral is of the general form $\exp \left(-\int_{\Sigma} \mathscr{L}\right)$, where $\mathscr{L}$ is some local functional. If $\Sigma$ is given as a union of smaller pieces - in the case at hand these will be the plaquettes $w_{i}$-then we have

$$
\exp \left(-\int_{\Sigma} \mathscr{L}\right)=\prod_{i} \exp \left(-\int_{w_{i}} \mathscr{L}\right)
$$

Thus, the integrand in the path integral is a product of local factors, one for each $w_{i}$. This is a feature that we wish to preserve.

Since the continuum limit of the two dimensional theory requires a choice of measure (but not metric), it is natural to introduce a lattice version of the measure. We assign an area $\varrho_{i}$ to each $w_{i}$, in such a way that $\sum \varrho_{i}=\varrho$, the total area of $\Sigma$.

A given plaquette $w$, of area $\varrho_{w}$, is bounded by edges $x_{1}, x_{2}, \ldots, x_{n}$ (Fig. 1b). A lattice connection assigns to these edges group elements $U_{1}, U_{2}, \ldots, U_{n}$. The only gauge invariant quantity that can be made from these is the holonomy

$$
\mathscr{U}=U_{1} U_{2} \ldots U_{n} .
$$

More exactly, the conjugacy class of the holonomy is gauge invariant. The local factor associated with a plaquette must be a class function of the holonomy. Such a class function must be a linear combination of the group characters, which are a basis for the class functions. For every isomorphism class $\alpha$ of irreducible representations of $G$, let $\chi_{\alpha}(\mathscr{U})$ be the corresponding character (the trace of $\mathscr{U}$ in the $\alpha$ representation). Migdal's considerations suggest the local factors

$$
\Gamma\left(\mathscr{U}, \varrho_{w}\right)=\sum_{\alpha} \operatorname{dim} \alpha \cdot \chi_{\alpha}(\mathscr{U}) \cdot \exp \left(-\varrho_{w} c_{2}(\alpha) / 2\right),
$$


where the sum runs over all isomorphism classes of representations, $\operatorname{dim} \alpha$ is the dimension of the representation $\alpha$, and $c_{2}(\alpha)$ is the quadratic Casimir operator of the group $G$, associated with the quadratic form on $\mathscr{G}$ introduced earlier.

This formula obviously requires some explanation. First of all, let us consider the behavior as $\varrho \rightarrow 0$. One has

$$
\sum_{\alpha} \operatorname{dim} \alpha \cdot \chi_{\alpha}(\mathscr{U})=\delta(\mathscr{U}-1),
$$

where $\delta(\mathscr{U}-1)$ is the delta function supported at $\mathscr{U}=1$. This behavior is desirable because, if the lattice theory is to have a continuum limit, the holonomy around a loop of area $\varrho$ should approach 1 as $\varrho \rightarrow 0$. In particular, (2.43) explains the factor of $\operatorname{dim} \alpha$ in (2.42), which will play a pivotal role as we will see.

The main justification for (2.42), however, is that it leads to a theory invariant under subdivision. Let us now explain this point. Once one has picked a covering $X$ of $\Sigma$ by polygons, one can define the corresponding lattice approximation to the path integral:

$$
Z_{\Sigma, X}(\varrho)=\int \prod_{\gamma} d U_{\gamma} \prod_{i} \Gamma\left(\mathscr{U}_{i}, \varrho_{i}\right) .
$$

Here $d U_{\gamma}$ denotes Haar measure on $G$, normalized so that the volume of $G$ is 1 . (Note: in Sect. 4, a different normalization of the Haar measure will be used in connection with a more precise computation.) In (2.44), $\gamma$ runs over all edges and $i$ runs over all plaquettes. We now wish to show that with the particular choice (2.42) for the local factors $\Gamma$, the partition function (2.44) is in fact independent of $X$.

To prove this, one must establish invariance under subdivision. So consider a case in which one of the polygons in $X$ is subdivided to get a finer covering $X^{\prime}$. For definiteness we consider (Fig. 1c) the case in which a square is subdivided into two triangles. Let $\varrho_{0}, \varrho^{\prime}$, and $\varrho^{\prime \prime}$ be the area of the square and of the two triangles (so $\left.\varrho^{\prime}+\varrho^{\prime \prime}=\varrho_{0}\right)$. The factor associated with the square is

$$
\Gamma=\sum_{\alpha} \operatorname{dim} \alpha \cdot \chi_{\alpha}\left(U_{1} U_{2} U_{3} U_{4}\right) \cdot \exp \left(-\varrho_{0} c_{2}(\alpha) / 2\right) .
$$

When the square is subdivided into two triangles, one introduces an extra edge with an extra group variable $V$. The product of the factors associated with the two triangles is

$$
\Gamma^{\prime} \Gamma^{\prime \prime}=\sum_{\alpha, \beta} \operatorname{dim} \alpha \cdot \operatorname{dim} \beta \cdot \chi_{\alpha}\left(U_{1} U_{2} V\right) \chi_{\beta}\left(V^{-1} U_{3} U_{4}\right) \exp \left(-\varrho^{\prime} c_{2}(\alpha) / 2-\varrho^{\prime \prime} c_{2}(\beta) / 2\right) .
$$

To prove that $Z_{\Sigma, X}=Z_{\Sigma, X^{\prime}}$, it suffices to prove that

$$
\int d V \Gamma^{\prime} \Gamma^{\prime \prime}=\Gamma
$$

since this means that integrating over the extra variable $V$ that appears in the definition of $Z_{\Sigma, X^{\prime}}$ will give back the integral representation of $Z_{\Sigma, X}$. But (2.47) is a consequence of the formula

$$
\int d V \chi_{\alpha}(A V) \chi_{\beta}\left(V^{-1} B\right)=\delta_{\alpha \beta} \cdot \frac{1}{\operatorname{dim} \alpha} \cdot \chi_{\alpha}(A B)
$$

(which is related to the orthonormality of the characters and their realization as functions on the group manifold; see [19, Chap. II, Theorem (4.5)]). 


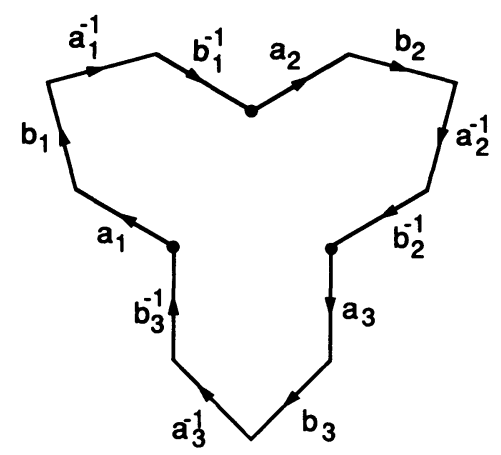

Fig. 2. An orientable surface of genus $g$ can be built by gluing together the sides of a $4 g$-sided polygon, shown here for $g=3$

Notice that in the verification of (2.46), it is essential that the dependence on $\varrho$ is exponential. However, the quadratic Casimir $c_{2}(\alpha)$ could have been replaced by any other function of $\alpha$. The use of the quadratic Casimir is appropriate because the continuum theory (2.3) that we are trying to reproduce has a Lagrangian density $\operatorname{Tr} f^{2}$ that is quadratic in $f$. In principle one could consider a continuum Lagrangian with $\operatorname{Tr} f^{2}$ replaced by $\operatorname{Tr} P(f)$, for any function $P(f)$; this would correspond to replacing $c_{2}(\alpha)$ by a more complicated function. To show that $c_{2}(\alpha)$ is the correct function that goes with the quadratic function in (2.3) is a standard computation (which we will not review here) using canonical quantization to construct the Hamiltonian, from which the $\varrho$ dependence of the continuum theory can be computed.

First Computations. One of the advantages of the discrete formulation, with invariance under subdivision, is that it makes it clear that the partition function $Z_{\Sigma}(\varrho)$ is computable. In fact, by considering a very fine lattice we would expect to get a result converging to the continuum theory, but invariance under subdivision means that one may in fact do the computation on an arbitrarily crude lattice.

Consider for simplicity the case that $\Sigma$ is an orientable surface of genus $g$. We can cover $\Sigma$ with a single $4 g$-sided polygon (Fig. 2 ). This corresponds to the wellknown description of the fundamental group of such a surface with $2 g$ generators $a_{i}, b_{j}, i, j=1 \ldots g$ and one relation $a_{1} b_{1} a_{1}^{-1} b_{1}^{-1} a_{2} b_{2} a_{2}^{-1} b_{2}^{-1} \ldots a_{g} b_{g} a_{g}^{-1} b_{g}^{-1}=1$. Denoting the holonomies around the generating cycles as $U_{i}$ and $V_{j}$, the YangMills partition function for a surface $\Sigma$ with this combinatorial description is

$$
Z_{\Sigma}(\varrho)=\sum_{\alpha} \operatorname{dim} \alpha \cdot e^{-\varrho c_{2}(\alpha) / 2} \cdot \int d U_{i} d V_{j} \chi_{\alpha}\left(U_{1} V_{1} U_{1}^{-1} V_{1}^{-1} \ldots U_{g} V_{g} U_{g}^{-1} V_{g}^{-1}\right) .(2
$$

The integrals over the $U_{i}$ and $V_{j}$ can be done one at a time using (2.48) and the related formula

$$
\int d U \chi_{\alpha}\left(A U B U^{-1}\right)=\frac{1}{\operatorname{dim} \alpha} \chi_{\alpha}(A) \chi_{\alpha}(B) .
$$

Every time one integrates over a $U_{i}$ or $V_{j}$ one gets a factor of $1 / \operatorname{dim} \alpha$ from (2.48) or (2.50). At the end one is left with $\chi_{\alpha}(1)=\operatorname{dim} \alpha$. Altogether one gets finally

$$
Z_{g}(\varrho)=\sum_{\alpha} \frac{e^{-\varrho c_{2}(\alpha) / 2}}{(\operatorname{dim} \alpha)^{2 g-2}}
$$

for the Yang-Mills partition function on an orientable surface of genus $g$. 
(a)

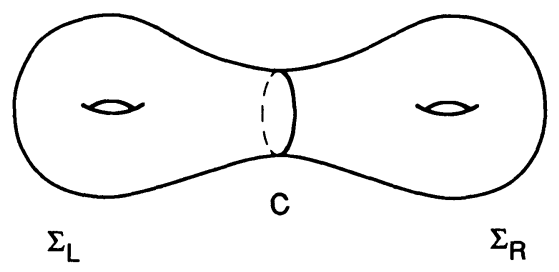

(b)

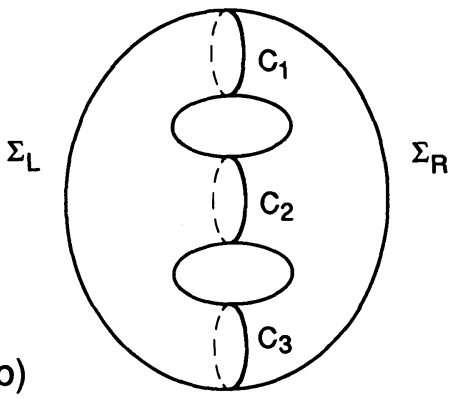

Fig. 3a, b. A surface $\Sigma$ "cut" on a circle $C$, dividing $\Sigma$ into pieces $\Sigma_{L}$ and $\Sigma_{R}(\mathbf{a})$. A cell decomposition of $C$ - which can be taken to have just one zero-cell and one one-cell - can be extended to a covering of $\Sigma$ by polygons. A more general case (b) in which $\Sigma$ is divided into $\Sigma_{L}$ and $\Sigma_{R}$ by cutting on several circles $C_{1}, \ldots, C_{n}$

Physical Hilbert Space and Axioms of Quantum Field Theory. It is useful, however, to rederive this formula from a slightly different point of view. Consider a surface $\Sigma$ "cut" along a circle $C$ to divide it into pieces $\Sigma_{L}$ and $\Sigma_{R}$, with areas $\varrho_{L}$ and $\varrho_{R}$ (Fig. 3). To introduce lattice gauge theory in a way compatible with this cutting, we first make a cell decomposition of $C$ - we may as well take the simplest one with a single vertex or zero-cell and a single edge or one-cell - and then extend this to coverings of $\Sigma_{L}$ and $\Sigma_{R}$ by polygons. If we do cover $C$ with a single one-cell, then in lattice gauge theory for this covering, there is precisely one group element $U$ attached to $C$ (more generally the holonomy $\mathscr{U}$ around $C$ will play the role of $U$ ). Other group variables $U_{L, \gamma}$ and $U_{R, \delta}$ are associated with edges that lie on $\Sigma_{L}$ or $\Sigma_{R}$.

It is convenient to carry out the integration over the gauge variables by integrating first over the $U_{L, \gamma}$ and $U_{R, \delta}$ and only at the end over $U$. The integral over $U_{R, \delta}$, for fixed $U$, defines a function of $U$ that we will call $\psi_{R}(U)$,

$$
\psi_{R}(U)=\int d U_{R, \delta} \prod_{w_{i} \in \Sigma_{R}} \Gamma\left(\mathscr{U}_{i}, \varrho_{i}\right) .
$$

The product on the right runs over the plaquettes making up $\Sigma_{R}$. Because of the reality of the weight factors in the lattice path integral, and the fact that the individual characters obey $\chi_{\alpha}(A)=\overline{\chi_{\alpha}\left(A^{-1}\right)}, \psi_{R}(U)$ obeys

$$
\overline{\psi_{R}(U)}=\psi_{R}\left(U^{-1}\right) \text {. }
$$

Similarly, the path integral on $\Sigma_{L}$ defines a function $\psi_{L}$ :

$$
\psi_{L}\left(U^{-1}\right)=\int d U_{L, \gamma} \prod_{w_{i} \in \Sigma_{L}} \Gamma\left(\mathscr{U}_{i}, \varrho_{i}\right) .
$$

We denote the right-hand side of $(2.54)$ as $\psi_{L}\left(U^{-1}\right)$ rather than $\psi_{L}(U)$ because of the reversal of orientation in regarding $C$ as the boundary of $\Sigma_{L}$ rather than $\Sigma_{R}$. Of course, $\psi_{L}$ also obeys (2.53). In addition, gauge invariance implies that $\psi_{L}$ and $\psi_{R}$ are class functions,

$$
\psi_{L}\left(A U A^{-1}\right)=\psi_{L}(U), \quad \psi_{R}\left(A U A^{-1}\right)=\psi_{R}(U) .
$$

To compute the partition function $Z_{\Sigma}(\varrho)$, we must now perform the final integral over $U$, to get

$$
Z_{\Sigma}(\varrho)=\int d U \psi_{L}\left(U^{-1}\right) \psi_{R}(U)=\int d U \overline{\psi_{L}(U)} \psi_{R}(U)
$$


It is natural now to introduce the Hilbert space $\mathscr{H}$ consisting of class functions on $G$ with inner product

$$
(f, g)=\int d U \overline{f(U)} g(U) .
$$

$\psi_{L}$ and $\psi_{R}$ are vectors in $\mathscr{H}$, and the partition function is

$$
Z_{\Sigma}(\varrho)=\left(\psi_{L}, \psi_{R}\right)
$$

There is also a very useful alternative way to formulate this. According to standard results in representation theory, the characters $\chi_{\sigma}(U)$ form an orthonormal basis of $\mathscr{H}$,

$$
\left.\int d U \overline{\chi_{\alpha}(U)} \chi_{\beta}(U)=\delta_{\alpha, \beta} ; \quad \text { and } \quad 1=\sum_{\alpha} \mid \chi_{\alpha}\right) \cdot\left(\chi_{\alpha} \mid\right.
$$

As a result,

$$
\left(\psi_{L}, \psi_{R}\right)=\sum_{\sigma}\left(\psi_{L}, \chi_{\sigma}\right) \cdot\left(\chi_{\sigma}, \psi_{R}\right)
$$

The factors on the right-hand side have convenient path integral representations, in which one integrates over all variables on $\Sigma_{L}$ or $\Sigma_{R}$ including the boundary values. For instance,

$$
\left(\chi_{\sigma}(U), \psi_{R}(U)\right)=\int d U \int d U_{R, \delta} \prod_{w_{i} \in \Sigma_{R}} \Gamma\left(\mathscr{U}_{i}, \varrho_{i}\right) \cdot \overline{\chi_{\sigma}(U)}
$$

More generally, consider a Riemann surface $\Sigma$ of area $\varrho$ with a boundary consisting of circles $C_{1}, \ldots, C_{n}$, and let $U_{1}, \ldots, U_{n}$ be the holonomies about these circles. For every labeling of the circles by representations $\alpha_{1}, \ldots, \alpha_{n}$, one defines a partition function $Z_{\Sigma}\left(\varrho ; \alpha_{1}, \ldots, \alpha_{n}\right)$ by modifying the original definition of the lattice path integral to include a factor of $\overline{\chi_{\alpha_{i}}\left(U_{i}\right)}$ for each $i$ :

$$
Z_{\Sigma}\left(\varrho ; \alpha_{1}, \ldots, \alpha_{n}\right)=\int \prod_{\gamma} d U_{\gamma} \prod_{i} \Gamma\left(\mathscr{U}_{i}, \varrho_{i}\right) \cdot \prod_{i=1}^{n} \overline{\chi_{\alpha_{i}}\left(U_{i}\right)} .
$$

By arguments along the lines that we have already given, it is possible to formulate a general cutting law (which amounts to the axioms of what one might call "areapreserving quantum field theory" in a sense analogous to axiomatizations of conformal and topological field theory in [7] and [20]). If a surface $\Sigma$ is cut into pieces $\Sigma_{\mu}, \mu=1, \ldots, k$, of areas $\varrho_{\mu}$, by cutting on circles $C_{i}, i=1, \ldots, n$ (Fig. 4), then the partition function of $\Sigma$ can be computed as follows. Pick orientations of the $C_{i}$ and label them by representations $\alpha_{i}$. $\left(C_{i}\right.$ with opposite orientation is then labeled by $\overline{\alpha_{i}}$. The partition function of $\Sigma_{\mu}$ with the induced labeling of its boundary will be called $Z_{\Sigma_{\mu}}\left(\varrho_{\mu} ;\{\alpha\}\right)$. The partition function of $\Sigma$ is then

$$
Z_{\Sigma}(\varrho)=\sum_{\{\alpha\}} \prod_{\mu} Z_{\Sigma_{\mu}}\left(\varrho_{\mu} ;\{\alpha\}\right)
$$

The proof is a repetition of the reasoning that led to the special case (2.58).

Relation to Canonical Quantization. The structure that we have found - associating a Hilbert space $\mathscr{H}$ with every circle $C$, with the factorization law (2.63) - is precisely the structure that is predicted by formal canonical quantization of the Lagrangian (2.3). Indeed, standard considerations of canonical quantization predict that $\mathscr{H}$ should be the Hilbert space of class functions that we have just found.

In Yang-Mills theory in $d$ dimensions, the physical Hilbert space associated with a $d-1$ manifold $Y$ (endowed with a $G$ bundle $E$ ) is always $L^{2}(\mathscr{A} / \hat{G})$, where $\mathscr{A}$ 


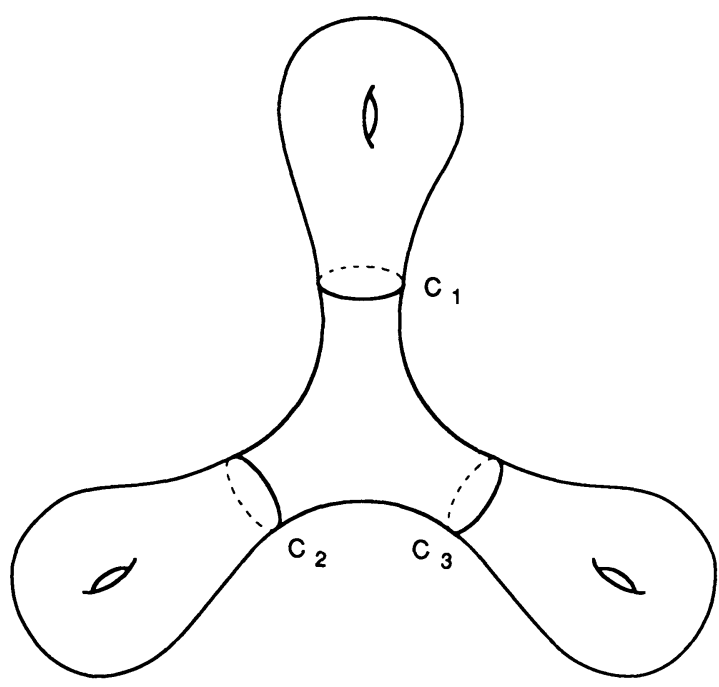

Fig. 4. A more general cutting of a surface $\Sigma$ on circles $C_{i}$; the path integral can be evaluated by assigning a representation $\alpha_{i}$ to each $C_{i}$, evaluating the path integrals over the components of $\Sigma$, and summing over the $\alpha_{i}$

is the space of connections on $E$ and $\hat{G}=\operatorname{Maps}(Y, G)$. In one dimension, as the only gauge invariant of a connection is the conjugacy class of the monodromy, $\mathscr{A} / \hat{G}$ is the space of conjugacy classes in $G$, and therefore $L^{2}(\mathscr{A} / \hat{G})$ is the Hilbert space of class functions that we have found above. As we have already noted, the $\varrho$ dependence in our formulas is derived in the canonical formalism by showing that the Hamiltonian - as an operator on $L^{2}(\mathscr{A} / \hat{G})$ - is a multiple of the quadratic Casimir operator.

Moreover, since the Lagrangian (2.3) is invariant under the group of areapreserving diffeormorphisms, and since every diffeomorphism of $C$ arises as the restriction of an area-preserving diffeomorphism of a neighborhood of $C$, the formal reasoning predicts that the group $\operatorname{diff} C$ of diffeomorphisms of $C$ should act on $\mathscr{H}$. It is indeed easy to describe the action of $\operatorname{diff} C$. An orientation-preserving diffeomorphism leaves unchanged the holonomy $U$ around $C$ and acts therefore as the identity on any function $\psi(U)$. On the other hand, an orientation-reversing diffeomorphism maps $U$ to $U^{-1}$ and so maps $\psi(U)$ to $\psi\left(U^{-1}\right)$. On the basis of $\mathscr{H}$ given by the characters $\chi_{\alpha}(U)$, the action of an orientation-reversing diffeomorphism is

$$
\chi_{\alpha}(U) \rightarrow \chi_{\alpha}\left(U^{-1}\right)=\chi_{\bar{\alpha}}(U),
$$

where $\bar{\alpha}$ is the representation dual or complex conjugate to $\alpha$.

\subsection{More Calculations}

In this subsection, we will redo the calculation of $Z_{\Sigma}(\varrho)$ for $\Sigma$ an orientable surface of genus $g>1$. Then we will consider some additional cases.

An orientable surface of genus $g>1$ can be decomposed into $2 g-2$ three holed spheres by cutting, as in Fig. $5 \mathrm{a}$, on $3 g-3$ circles. To compute its partition function 
(a)

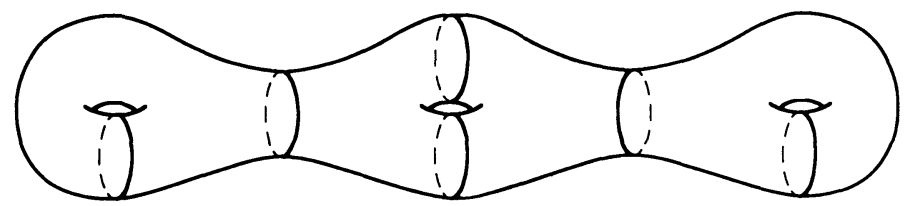

(b)

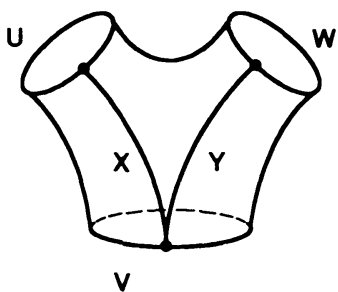

(c)

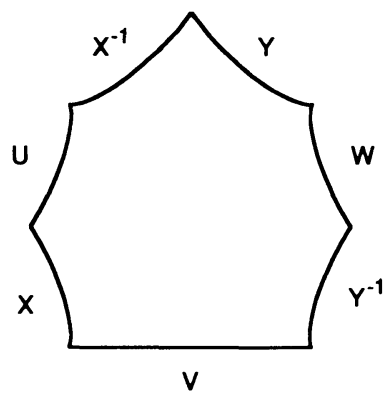

(d)

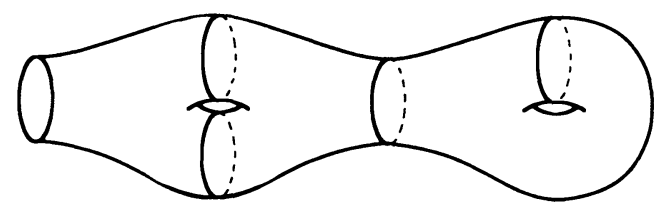

Fig. 5a-d. An orientable surface of genus $g>1$ can be decomposed into $2 g-2$ three holed spheres by cutting on $3 g-3$ circles a. A three holed sphere can be conveniently covered with a single polygon 6; an unwrapped version of this is in c. An orientable surface of genus $g$ with $n$ holes can be made by gluing together $2 g-2+n$ three holed spheres, as illustrated for $g=2$ and $n=1$ in d

using (2.63), the main point is to understand the partition function $Z_{3}(\varrho ; \alpha, \beta, \gamma)$ of a three holed sphere of area $\varrho_{0}$ and boundary components labeled by representations $\alpha, \beta$, and $\gamma$. To do this, we pick a convenient covering of the three holed sphere by a single polygon, as sketched in Fig. 5b,c. Denoting the holonomies around the three holes as $U, V$, and $W$, and the gauge variables in the interior of the three holed sphere as $X, Y$, the definition (2.62) of the partition function of a surface with labeled boundary reduces in this case to

$$
\begin{aligned}
& Z_{3}\left(\varrho_{0} ; \alpha, \beta, \gamma\right)=\sum_{\sigma} \operatorname{dim} \sigma e^{-\varrho_{0} c_{2}(\sigma) / 2} \\
& \quad \times \int d X d Y d U d V d W \chi_{\sigma}\left(X^{-1} U X V Y^{-1} W Y\right) \cdot \overline{\chi_{\alpha}(U)} \overline{\chi_{\beta}(V)} \overline{\chi_{\gamma}(W)} .
\end{aligned}
$$

Using (2.50) to do the integrals over $X$ and $Y$, and then using the orthonormality (2.59) of the characters to integrate over $U, V$, and $W$, we get

$$
Z_{3}(\varrho ; \alpha, \beta, \gamma)=\frac{e^{-\varrho c_{2}(\alpha) / 2}}{\operatorname{dim} \alpha} \cdot \delta_{\alpha, \beta, \gamma} .
$$

Here $\delta_{\alpha, \beta, \gamma}$ is one for $\alpha=\beta=\gamma$ and zero otherwise.

Now we can compute the partition function for an orientable surface of genus $g$. Cutting as in Fig. 5a, we then label the $3 g-3$ circles $C_{i}$ by representations $\alpha_{i}$, and perform the product and sum in (2.63). Because of the $\delta_{\alpha, \beta, \gamma}$ in (2.66), a nonzero 


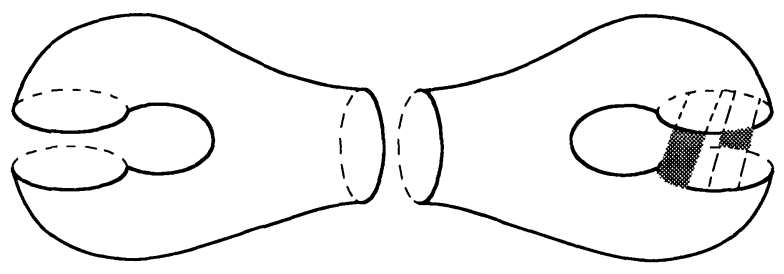

Fig. 6. The connected sum of a surface of genus one and a Klein bottle can be made by gluing together two three holed spheres along their boundary circles but an orientation-reversing diffeomorphism of one of the boundary circles, which is crudely sketched here on the right, is made before the gluing

contribution only arises when all $\alpha_{i}$ are equal. Each of the $2 g-2$ three holed spheres gives a factor of $1 / \operatorname{dim} \alpha$. So we get again

$$
Z_{g}(\varrho)=\sum_{\alpha} e^{-\varrho c_{2}(\alpha) / 2} \frac{1}{(\operatorname{dim} \alpha)^{2 g-2}} .
$$

We also will need the corresponding formula for the partition function of a surface of genus $g$ with $n$ holes labeled by representations $\alpha_{1}, \ldots, \alpha_{n}$. Such a surface can be made by gluing together $2 g-2+n$ three holed spheres, as in Fig. $5 \mathrm{~d}$. The partition function vanishes unless the $\alpha_{i}$ are all equal, because of the $\delta_{\alpha, \beta, \gamma}$ in (2.66), while if they are equal one gets $n$ extra factors of $1 /(\operatorname{dim} \alpha)$ compared to the previous computation. So the partition function in this case is

$$
Z_{g}\left(\varrho ; \alpha_{1}, \ldots, \alpha_{n}\right)=\delta_{\alpha_{1}, \ldots, \alpha_{n}} \cdot e^{-\varrho c_{2}\left(\alpha_{1}\right) / 2} \cdot \frac{1}{(\operatorname{dim} \alpha)^{2 g-2+n}} .
$$

Unorientable Surfaces. We now want to consider unorientable surfaces. First we consider the easy case of a connected sum of an orientable surface of genus $g$ with $s$ copies of the Klein bottle. Such a surface $\Sigma_{g, s}$ can be built as a union of $2 g-2+2 s$ three holed spheres, glued together along their boundary components, but one makes orientation-reversing diffeomorphisms of $s$ of the boundary components prior to the gluing (Fig. 6). According to (2.64), an orientation-reversing diffeomorphism of the boundary changes the label from $\alpha$ to $\bar{\alpha}$. Because of the $\delta_{\alpha, \beta, \gamma}$ in the amplitude for a three holed sphere, this actually means that, when we use the formula (2.63) to evaluate the partition function of $\Sigma_{g, s}$, a nonzero contribution arises only for representations such that $\alpha=\bar{\alpha}$, that is, representations that are isomorphic to their complex conjugates. Once one restricts to such a representation, the contribution to the partition function of $\Sigma_{g, s}$ is the same as it would be for an orientable surface of the same Euler characteristic, and so the partition function is

$$
Z_{g, s}(\varrho)=\sum_{\alpha=\bar{\alpha}} \frac{e^{-\varrho c_{2}(\alpha) / 2}}{(\operatorname{dim} \alpha)^{2 g-2+2 s}} .
$$

We now want to consider connected sums with copies of $R P^{2}$. In so doing we will need the following formula from the theory of compact groups:

$$
\int d U \chi_{\alpha}\left(U^{2}\right)=f_{\alpha},
$$

where $f_{\alpha}=1$ if there is a symmetric invariant bilinear form $\alpha \otimes \alpha \rightarrow \mathbb{C}, f_{\alpha}=-1$ if there is an antisymmetric invariant bilinear form $\alpha \otimes \alpha \rightarrow \mathbb{C}$, and $f_{\alpha}=0$ if there is no 
(a)

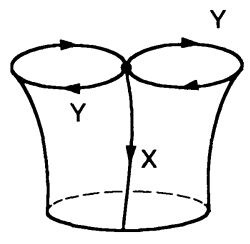

U (b)

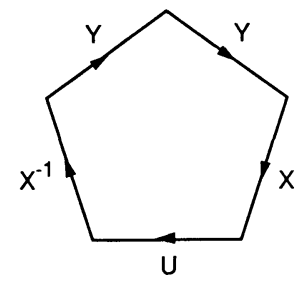

Fig. 7a, b. A copy of $R P^{2}$ with one hole can be built by gluing together the lines labeled $Y$ in a. Cutting along the line labeled as $X$, this can be unwrapped to give $\mathbf{b}$

invariant bilinear form $\alpha \otimes \alpha \rightarrow \mathbb{C}$ (see [19, Chap. II, Proposition (6.8)]). In particular, $f_{\alpha}=0$ unless $\alpha=\bar{\alpha}$, and if $\alpha=\bar{\alpha}$ then $f_{\alpha}^{2}=1$.

A connected sum with copies of $R P^{2}$ cannot be built by gluing together three holed spheres, so we will have to do some work. Let $\Sigma^{\prime}$ be a copy of $R P^{2}$ with a ball removed. Thus, the boundary of $\Sigma^{\prime}$ consists of a single circle; let $U$ denote the holonomy about this circle. Label the boundary of $\Sigma^{\prime}$ by a representation $\alpha$, and let $\varrho^{\prime}$ be the area of $\Sigma^{\prime}$. We want to compute the partition function $Z^{\prime}\left(\varrho^{\prime} ; \alpha\right)$ for this situation. $\Sigma^{\prime}$ can be covered with one polygon as shown in Fig. 7. This involves introducing internal integration variables which are labeled as $X$ and $Y$ in the figure. The general definition (2.62) of the partition function of a surface with labeled boundary gives in this case

$$
Z^{\prime}\left(\varrho^{\prime} ; \alpha\right)=\sum_{\beta} \operatorname{dim} \beta \cdot e^{-\varrho^{\prime} c_{2}(\beta) / 2} \int d X d Y d U \chi_{\beta}\left(U X Y^{2} X^{-1}\right) \cdot \overline{\overline{\chi_{\alpha}}(U)} .
$$

Using (2.50) to integrate over $X$ and then integrating over $Y$ with (2.70) and integrating over $U$ using the orthonormality of the characters, we get

$$
Z^{\prime}\left(\varrho^{\prime} ; \alpha\right)=f_{\alpha} e^{-\varrho^{\prime} c_{2}(\alpha) / 2} .
$$

Now let $\Sigma_{g, s, r}$ be the connected sum of a surface of genus $g$ with $s$ copies of the Klein bottle and $r$ copies of the projective plane. Such a surface can be built by gluing together $2 g-2+2 s+r$ three holed spheres and $r$ one holed $R P^{2}$ 's. The evaluation of (2.63) gives for the partition function

$$
Z_{g, s, r}(\varrho)=\sum_{\alpha} \frac{f_{\alpha}^{r} e^{-\varrho c_{2}(\alpha) / 2}}{(\operatorname{dim} \alpha)^{2 g-2+2 s+r}} .
$$

(This is written for $r>0$. If $r=0$ and $s>0$, the sum must be restricted to $\alpha$ such that $\alpha=\bar{\alpha}$.) Since $f_{\alpha}^{3}=f_{\alpha}$ for all $\alpha$, this formula is compatible with the fact that the connected sum of three copies of $R P^{2}$ is the same as the connected sum of $R P^{2}$ and a Klein bottle.

Twisted External States. We will now discuss the analog in Yang-Mills theory of permitting "punctures" in rational conformal field theory. We consider a surface $\Sigma$ with boundary consisting of a circle $C$ (the generalization to the case in which the boundary has several components will be immediate). Let $\Theta$ be some conjugacy class in $G$. We want to calculate the Yang-Mills path integral over all connections on $\Sigma$ for which the holonomy around $C$ is in the conjugacy class $\Theta$. We shall denote the partition function integrated over connections with this property as $Z_{\Sigma}(\varrho ; \Theta)$. This partition function can be computed as follows. If $U$ denotes the holonomy of the connection around $C$, then according to our above discussion, the path integral 
on $\Sigma$, as a function of $U$, defines a vector $\psi(U)$. Previously, picking a representation $\alpha$ of $G$, we paired $\psi$ with $\chi_{\alpha}(U)$ to define

$$
Z_{\Sigma}(\varrho ; \alpha)=\left(\chi_{\alpha}(U), \psi(U)\right) .
$$

Now we simply want to replace the wave function $\chi_{\alpha}(U)$ by a state $\left.\mid \Theta\right)$ with delta function support on the conjugacy class $\Theta$, and define

$$
Z_{\Sigma}(\varrho ; \Theta)=(\Theta, \psi) \text {. }
$$

Using the orthonormality of the characters, we have

$$
\left.\left.\mid \Theta)=\sum_{\alpha} \mid \alpha\right)(\alpha \mid \Theta)=\sum_{\alpha} \mid \alpha\right) \cdot \overline{\chi_{\alpha}(\Theta)} .
$$

Actually, the notion of a "delta function supported on the conjugacy class $\Theta$ " is somewhat imprecise, since the normalization of such a delta function depends on the choice of a measure on the space of conjugacy classes. We have made a particular choice in (2.76); another choice would change $\mid \Theta)$ to $f(\Theta) \mid \Theta)$, where $f$ is some class function. (In Sect. 4, we will discuss more critically the appropriate measure on the space of class functions.)

We thus have

$$
Z_{\Sigma}(\varrho ; \Theta)=\sum_{\alpha} Z_{\Sigma}(\varrho ; \alpha) \cdot \chi_{\alpha}(\Theta) .
$$

More generally, if $\Sigma$ has $n$ boundary components, and we integrate over connections whose holonomy around the $i^{\text {th }}$ boundary component is in a conjugacy class $\Theta_{i}$, then the corresponding partition function is

$$
Z_{\Sigma}\left(\varrho ; \Theta_{1}, \ldots, \Theta_{n}\right)=\sum_{\alpha_{1}, \ldots, \alpha_{n}} Z_{\Sigma}\left(\varrho ; \alpha_{1}, \ldots, \alpha_{n}\right) \cdot \chi_{\alpha_{1}}\left(\Theta_{1}\right) \cdot \ldots \cdot \chi_{\alpha_{n}}\left(\Theta_{n}\right) .
$$

But the right-hand side of (2.78) has been evaluated in (2.68). So in fact we get

$$
Z_{\Sigma}\left(\varrho ; \Theta_{1}, \ldots, \Theta_{n}\right)=\sum_{\alpha} e^{-\varrho c_{2}(\alpha) / 2} \frac{1}{(\operatorname{dim} \alpha)^{2 g-2+n}} \cdot \prod_{i=1}^{n} \chi_{\alpha}\left(\Theta_{i}\right) .
$$

If the $\mid \Theta_{i}$ ) are transformed as indicated at the end of the last paragraph, this formula will transform as

$$
Z_{\Sigma}\left(\varrho ; \Theta_{1}, \ldots, \Theta_{n}\right) \rightarrow Z_{\Sigma}\left(\varrho ; \Theta_{1}, \ldots, \Theta_{n}\right) \cdot \prod_{i=1}^{n} f\left(\Theta_{i}\right)
$$

for some class function $f$.

One can easily generalize (2.79) to the case of an unorientable surface, but we will forgo this.

Synthesis. We have noted in Sect. 2.1 that two dimensional Yang-Mills theory is closely related to the large $k$ limit of three dimensional Chern-Simons theory and thus to the large $k$ limit of two dimensional current algebra. The reader familiar with the latter subject will notice that many facts familiar in rational conformal field theory (and explained, for the most part, in [5]) have much simpler echos in the above discussion. The fact that the Hilbert space $\mathscr{H}$ associated with every circle has one basis vector for every irreducible representation of $G$ is the large $k$ limit of what happens in the conformal field theory problem where (at level $k$ ) only a certain finite list of representations is permitted. The fact that the amplitude for a three holed sphere is "diagonal" $[(2.66)$ vanishes unless $\alpha=\beta=\gamma]$ is the large $k$ limit 


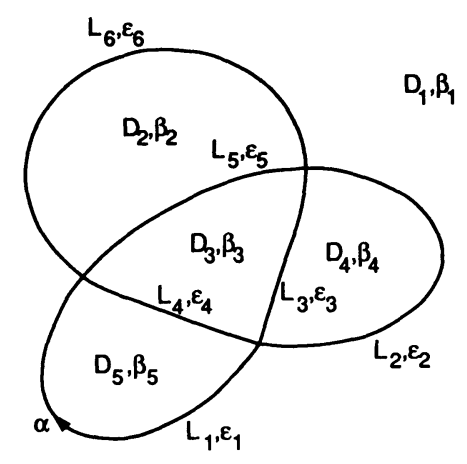

Fig. 8. A closed immersed loop drawn on a two sphere, which has been projected to the plane. The loop is labeled by a representation $\alpha$. The plane is divided in regions $D_{\lambda}$ of area $\varrho_{\lambda}$. Each region $D_{i}$ is labeled by a representation $\beta_{i}$. The loop is divided by its self-crossings into segments $L_{\sigma}$. In the course of the computation these acquire labels $\varepsilon_{\sigma}$

of Verlinde's diagonalization of the fusion rules. And indeed the formulas that we obtained for the Yang-Mills partition function are closely related to Verlinde's formulas, as we will see presently.

\subsection{Comparison to IRF Models}

This subsection will be devoted to a matter that is somewhat removed from our main subject. We want to briefly indicate the relation of two dimensional YangMills theory to IRF models of statistical mechanics [21].

This connection arises when one considers "Wilson lines." We pick an immersed circle $C$ on a surface $\Sigma$ of area $\varrho$ (we will only consider the case that the singularities of $C$ are all simple crossings) and associate with it a representation $\alpha$. Given a connection $A$ on $\Sigma$, we form the holonomy $V=P \exp \oint_{C} A$ of the connection $A$ around $C$ and take its character in the $\alpha$ representation to get a functional of $A$ :

$$
W_{C, \alpha}(A)=\chi_{\alpha}(V) \text {. }
$$

More generally, we consider several circles $C_{i}$, labeled by representations $\alpha_{i}$, and define the corresponding functionals $W_{C_{i}, \alpha_{i}}(A)=\chi_{\alpha_{i}}\left(V_{i}\right)$, with $V_{i}$ the holonomy around $C_{i}$. Then we wish to calculate the path integral with an insertion of these factors

$$
Z_{\Sigma}\left(\varrho ; C_{i}, \alpha_{i}\right)=\int D A \exp (-I(A)) \prod_{i} W_{C_{i}, \alpha_{i}}(A) .
$$

Physically, the $W_{C, \alpha}(A)$ are called Wilson line functionals, and the ratio $Z_{\Sigma}\left(\varrho ; C_{i}, \alpha_{i}\right) / Z$ is called the expectation value of the product of Wilson lines. Such expectation values in two dimensional gauge theories have been computed from several points of view [15, 22-24]. (L. Gross has suggested, in connection with [24], that this problem is related to quantum groups, and this may be connected to the discussion here.) We will formulate this in a way that exhibits the relation to IRF models which also appear, after a much more difficult analysis, in computing Wilson line expectation values in three dimensional Chern-Simons theory [25]. For convenience, we will consider first the case that $\Sigma$ has genus zero.

As indicated in Fig. 8, the loops $C_{i}$ are divided by their self-intersections into segments $L_{\sigma}$ that are topologically one-cells. (We will not consider the case in 
which one of the $C_{i}$ does not intersect itself or the others, in which event this assertion is not true. The required generalization is not difficult, however.) $\Sigma$ is divided by the loops into regions $D_{\lambda}$ of areas $\varrho_{\lambda}$. By our general discussion, in formulating lattice gauge theory, we can use any division of $\Sigma$ into polygons as the starting point; we shall take the division in which the $L_{\sigma}$ are the edges and the $D_{\lambda}$ are the plaquettes. Consequently, we introduce a group-valued integration variable $U_{\sigma}$ for each edge. In the simplicial version of the path integral, we must label each $D_{\lambda}$ by a representation $\beta_{\lambda}$. The local factor associated to $D_{\lambda}$ is

$$
\Gamma\left(D_{\lambda}\right)=\sum_{\beta_{\lambda}} \operatorname{dim} \beta_{\lambda} \cdot e^{-e_{\lambda} c_{2}\left(\beta_{\lambda}\right) / 2} \cdot \chi_{\beta_{\lambda}}\left(\mathscr{U}_{\lambda}\right),
$$

where $U_{\lambda}$ is the holonomy of the lattice connection around the boundary of $D_{\lambda}$. The lattice version of the path integral is

$$
Z_{\Sigma}\left(\varrho ; C_{i}, \alpha_{i}\right)=\sum_{\left\{\beta_{\lambda}\right\}} \int \prod_{\gamma} d U_{\gamma} \prod_{\lambda} \Gamma\left(D_{\lambda}\right) \prod_{i} W_{C_{i}, \alpha_{i}}\left(V_{i}\right),
$$

where of course the holonomies $V_{i}$ around the $C_{i}$ are computed using the lattice connection.

We want to show that after performing the integration over the $U_{\gamma},(2.84)$ reduces to a lattice statistical mechanics expression (of a type familiar in IRF models) in which the statistical variables are the $\beta_{\lambda}$, associated with the $D_{\lambda}$, and certain discrete variables $\varepsilon_{\sigma}$ associated with the edges $L_{\sigma}$, which will appear presently. The Boltzmann weights in this statistical model will be a product of local weights associated with the crossings in Fig. 8. To extract such a picture, we notice first that each $U_{\gamma}$ appears in three places in the integrand in (2.84). It appears in $W_{C_{l}, \alpha_{l}}$ - where $C_{l}$ is the circle containing $L_{\gamma}$-and in $\Gamma\left(D_{\lambda}\right)$ and $\Gamma\left(D_{\lambda^{\prime}}\right)$, where $D_{\lambda}$ and $D_{\lambda^{\prime}}$ are the two regions bordered by $L_{\gamma}$. The part of the integral that contains $U_{\sigma}$ is therefore

$$
\int d U_{\sigma} \chi_{\alpha_{l}}(X U) \chi_{\beta_{\lambda}}(Y U) \chi_{\beta_{\lambda}{ }^{\prime}}(Z U)
$$

for some $X, Y$, and $Z$. If write $R_{\alpha}(U)_{i^{\prime}}^{i}$ for the matrix representating $U$ in the $\alpha$ representation, then we have

$$
\chi_{\alpha}(X U)=R_{\alpha}(X)_{i}^{i^{\prime}} R_{\alpha}(U)_{i^{\prime}}^{i}
$$

and after using this three times, we see that to compute the integral $(2.85)$ it is enough to know

$$
\int d U R_{\alpha_{l}}(U)_{i^{\prime}}^{i} R_{\beta_{\lambda}}(U)_{j^{\prime}}^{j} R_{\beta_{\lambda^{\prime}}}(U)_{k^{\prime}}^{k}
$$

To make a general statement about (2.87), consider for every three representations $\alpha, \alpha^{\prime}, \alpha^{\prime \prime}$ of $G$ the finite dimensional vector space $\mathscr{H}_{\alpha, \alpha^{\prime}, \alpha^{\prime \prime}}=\left(\alpha \otimes \alpha^{\prime} \otimes \alpha^{\prime \prime}\right)^{G}$. Let $d_{\alpha, \alpha^{\prime}, \alpha^{\prime \prime}}$ be its dimension. A vector $\varepsilon \in \mathscr{H}_{\alpha, \alpha^{\prime}, \alpha^{\prime \prime}}$ can be represented by an invariant tensor

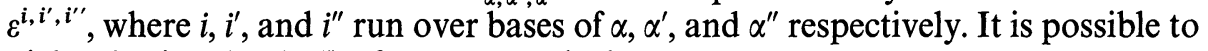
pick a basis $\varepsilon_{\mu}\left(\alpha, \alpha^{\prime}, \alpha^{\prime \prime}\right)$ of $\mathscr{H}_{\alpha, \alpha^{\prime}, \alpha^{\prime \prime}}$ such that

$$
\int d U R_{\alpha}(U)_{i^{\prime}}^{i} R_{\alpha^{\prime}}(U)_{j^{\prime}}^{j} R_{\alpha^{\prime \prime}}(U)_{k^{\prime}}^{k}=\sum_{\mu=1}^{d_{\alpha, \alpha^{\prime}, \alpha^{\prime \prime}}} \varepsilon_{\mu}\left(\alpha, \alpha^{\prime}, \alpha^{\prime \prime}\right)^{i j k} \bar{\varepsilon}_{\mu}\left(\alpha, \alpha^{\prime}, \alpha^{\prime \prime}\right)_{i^{\prime} j^{\prime} k^{\prime}} .
$$

Henceforth, for each trio of representations $\alpha, \alpha^{\prime}, \alpha^{\prime \prime}$, we fix a basis with this property.

We can integrate over all of the $U_{\gamma}$ 's in (2.84) by using (2.88) once for each $\gamma$. At the end of this, of course, each edge $L_{\gamma}$ is labeled by an appropriate $\varepsilon_{\gamma}$ [which ranges 


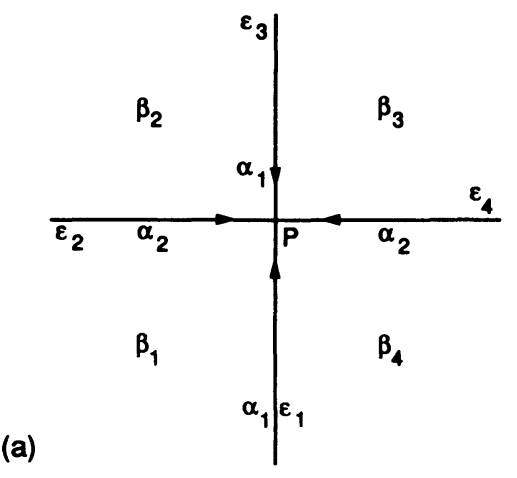

(b)

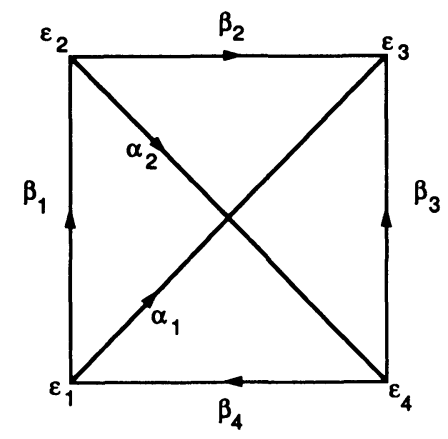

Fig. 9a, b. The configuration near a particular vertex $P$ is sketched in a. The data present is that which is required to single out an IRF configuration, often drawn as in b

over a finite set consisting of a basis of the appropriate $\mathscr{H}$ chosen to obey (2.88)]. At this point the variables over which we must sum are discrete variables: the $\beta_{\lambda}$ and $\varepsilon_{\gamma}$ by which the plaquettes and edges in Fig. 8 are labeled, and the indices $i, j, k$ which have proliferated in equations such as (2.88).

The summation over these indices can be carried out in a convenient way. The point is that the indices in (2.88) appear in a "factorized" form. One factor, $\varepsilon_{\mu}$, contains indices that "live" at one end of the edge $L_{\gamma}$, and the other factor, $\bar{\varepsilon}_{\mu}$, has indices that "live" at the other end. Thus, each index is naturally associated with a particular end of an edge or in other words with a particular vertex $P$. Thus, the problem of summing over the indices is a local problem that must be solved for each vertex. This problem depends only on the labeling of the regions $D_{\lambda}$ and edges $L_{\gamma}$ adjoining $P$.

Every vertex $P_{Q}$ (Fig. 9) is at a crossing of two circles, which are labeled by representations $\alpha_{i}$. The four edges $L_{\gamma}$ that meet at $P_{e}$ are each labeled by an $\varepsilon_{\gamma}$. The four plaquettes $D_{\lambda}$ with corners at $P_{\varrho}$ are labeled by representations $\beta_{\lambda}$. After summing the product of the four $\varepsilon$ 's over all their indices, we get a local factor which we will call $G_{e}\left(\alpha_{i} ; \beta_{\lambda} ; \varepsilon_{\gamma}\right)$. (It is essentially the definition of the Wigner-Racah classical $6 j$ symbol, but we will not pause to spell this out.)

After integrating over the $U_{\gamma}$ 's and summing over the indices in the fashion described above, (2.84) reduces to a lattice statistical mechanics formula

$$
Z_{\Sigma}\left(\varrho ; C_{i}, \alpha_{i}\right)=\sum_{\beta_{\lambda}} \sum_{\varepsilon_{\gamma}} \prod_{\lambda} \operatorname{dim}\left(\beta_{\lambda}\right) \cdot e^{-\varrho_{\lambda} c_{2}\left(\beta_{\lambda}\right) / 2} \cdot \prod_{\varrho} G_{\varrho}\left(\alpha_{i} ; \beta_{\lambda} ; \varepsilon_{\gamma}\right)
$$

that is obviously reminiscent of IRF models.

The generalization of (2.89) to Wilson lines on a surface of higher genus is straightforward. The only difference is that the Wilson lines divide the surface into regions $D_{\lambda}$ that are not necessarily discs but more generally (in the orientable case, for simplicity) surfaces of genus $g_{\lambda}$ with $n_{\lambda}$ holes. In this case, according to (2.68), we need only replace the factors of $\operatorname{dim}\left(\beta_{\lambda}\right)$ in $(2.89)$ with $\left(\operatorname{dim}\left(\beta_{\lambda}\right)\right)^{2-2 g_{\lambda}-n_{\lambda}}$.

The elementary considerations that we have explained do not immediately shed much light on integrable IRF models [which have additional features, notably the spectral parameter, that are missing in (2.89)], but perhaps these considerations are a useful starting point for further attempts to derive IRF models from gauge theories. 


\section{Use of Verlinde's Formula}

Let us now return to the situation considered in the introduction. We recall that $\mathscr{M}$ is the moduli space of flat $G$ bundles on a surface $\Sigma$. Once a complex structure is picked on $\Sigma, \mathscr{M}$ can be regarded as the moduli space of holomorphic bundles on $\Sigma$. Over $\mathscr{M}$ is a holomorphic line bundle $\mathscr{L}$, whose first Chern class is represented in de Rham cohomology by the symplectic structure $\omega$ of $\mathscr{M}$. That symplectic structure is given by the formula

$$
\omega=\frac{1}{4 \pi^{2}} \int_{\Sigma} \operatorname{Tr} \delta A \wedge \delta A .
$$

This formula means that if $a, b$ are $\operatorname{ad}(E)$-valued one forms representing tangent vectors to $\mathscr{M}$, then

$$
\omega(a, b)=\frac{1}{4 \pi^{2}} \int_{\Sigma} \operatorname{Tr} a \wedge b .
$$

Verlinde [5] gave a formula for the dimension of the space of conformal blocks in conformal field theory, and this can be interpreted as a formula for the dimension of $H^{0}\left(\mathscr{M}, \mathscr{L}^{\otimes k}\right)$. In this section we will use the Verlinde formula to get results which we will compare both with the Yang-Mills theory studied in the last section and with the treatment via Reidemeister torsion that will occupy Sect. 4. We will be rather brief and refer to [5] for more detail. Though Verlinde's formula is completely general, we will for simplicity focus on the cases $G=S U(2)$ and $G=S O(3)$. For related discussions, see $[10,26]$.

Verlinde's formula is

$$
\operatorname{dim} H^{0}\left(\mathscr{M}, \mathscr{L}^{\otimes k}\right)=\sum_{\alpha} \frac{1}{S_{0, \alpha}^{2 g-2}} .
$$

Here $\alpha$ runs over representations of $G$ which are highest weights of integrable representations of the loop group $\mathscr{L} G$ at level $k, S_{\alpha \beta}$ is a matrix that arises in the theory of loop groups ${ }^{4}$, and " 0 " denotes the trivial representation. For instance, in the case of $G=S U(2)$, the integrable representations are the representations of dimension $1,2, \ldots, k+1$. We use the letter $s, 0 \leqq s \leqq k$, to refer to the representation of dimension $s+1$. One has

$$
S_{i j}=\sqrt{\frac{2}{k+2}} \sin \frac{\pi(i+1)(j+1)}{k+2},
$$

and hence

$$
\operatorname{dim} H^{0}\left(\mathscr{M}, \mathscr{L}^{\otimes k}\right)=\left(\frac{k+2}{2}\right)^{g-1} \sum_{j=0}^{k} \frac{1}{\left(\sin \frac{\pi(j+1)}{k+2}\right)^{2 g-2}} .
$$

Since our goal is to obtain a formula for the volume of $\mathscr{M}$, we need to extract a term proportional to $k^{\mathrm{dim} \mathbf{c} M}=k^{3 g-3}$ for $k \rightarrow \infty$. A term that grows as fast as this arises only because $1 / \sin (\pi(j+1) /(k+2))$ is of order $k$ for large $k$ and fixed $j$ or fixed $k-j$.

\footnotetext{
${ }^{4}$ If $\chi_{\alpha}(\tau)$ is the character of the $\mathscr{L} G$ representation at level $k$ with highest weight $\alpha$, then $S$ is defined by the formula $\chi_{\alpha}(-1 / \tau)=\sum_{\beta} S_{\alpha \beta} \chi_{\beta}(\tau)$
} 
The two regions of $j \ll k$ and $k-j \ll k$ make equal contributions in (3.5). Assuming $j \ll k$, we get

$$
\frac{1}{\sin (\pi(j+1) /(k+2))} \sim k / \pi(j+1),
$$

so including both contributions, (3.5) becomes

$$
\operatorname{dim} H^{0}\left(\mathscr{M}, \mathscr{L}^{\otimes k}\right) \sim 2\left(\frac{k+2}{2}\right)^{g-1} \sum_{j=0}^{k}\left(\frac{k+2}{\pi(j+1)}\right)^{2 g-2} .
$$

This reduces to

$$
\operatorname{dim} H^{0}\left(\mathscr{M}, \mathscr{L}^{\otimes k}\right) \sim 2 \frac{k^{3 g-3}}{2^{g-1} \pi^{2 g-2}} \sum_{n=1}^{\infty} \frac{1}{n^{2 g-2}}=2 \frac{k^{3 g-3}}{2^{g-1} \pi^{2 g-2}} \zeta(2 g-2),
$$

with $\zeta(s)$ the Riemann zeta function.

According to the Riemann-Roch formula, the left-hand side of (3.7) is (provided the higher cohomology vanishes, which is true on general grounds at least for large enough $k$ )

$$
\operatorname{dim} H^{0}\left(\mathscr{M}, \mathscr{L}^{\otimes k}\right)=\left\langle e^{k c_{1}(\mathscr{L})} \operatorname{Td}(\mathscr{M}), \mathscr{M}\right\rangle,
$$

where $\operatorname{Td}(\mathscr{M})$ denotes the Todd class. For large $k,(3.9)$ gives

$$
\operatorname{dim} H^{0}\left(\mathscr{M}, \mathscr{L}^{\otimes k}\right) \sim \frac{k^{3 g-3}}{(3 g-3) !}\left\langle c_{1}(\mathscr{L})^{3 g-3}, \mathscr{M}\right\rangle .
$$

Now [as $c_{1}(\mathscr{L})$ is represented by the symplectic form $\omega$ in de Rham cohomology], $\left\langle c_{1}(\mathscr{L})^{3 g-3}, \mathscr{M}\right\rangle /(3 g-3)$ ! coincides with the volume $\operatorname{Vol}(\mathscr{M})$ that we aim to compute. Thus, (3.10) and (3.8) combine to give

$$
\operatorname{Vol}(\mathscr{M})=2 \cdot \frac{1}{\left(2 \pi^{2}\right)^{g-1}} \cdot \sum_{n=1}^{\infty} n^{-(2 g-2)}=2 \cdot \frac{\zeta(2 g-2)}{\left(2 \pi^{2}\right)^{g-1}}
$$

for $G=S U(2)$.

In Sect. 4, we will recover this result from the point of view of Reidemeister torsion. For the moment, let us compare it to our results in Sect. 2. There, we found that the partition function of Yang-Mills theory in genus $g\left(\right.$ at $\left.e^{2} \varrho=0\right)$ is

$$
Z=\zeta(2 g-2) \text {. }
$$

According to Sect. 2, the relation between the volume and $Z$ is to be

$$
\operatorname{Vol}(\mathscr{M})=Z \cdot 2 \cdot e^{\Delta v(2-2 g)}
$$

where 2, which is the order of the center of $S U(2)$, is the number of gauge symmetries of a generic $S U(2)$ flat connection, and $\Delta v$ is a constant, explained in Eq. (2.7), that depends on the renormalization convention used in defining $Z$. We see that (3.11) and (3.12) are in accord with this relation, with $e^{\Delta v}=\sqrt{2 \pi^{2}}$. (The value of $\Delta v$ is of limited significance. By rescaling the Haar measures and local factors used in Sect. 2.3, whose normalization depended on an arbitrarily chosen constant, we could have obtained a different theory, still invariant under subdivision, but leading to a different $\Delta v$.) 
Now we want to generalize this to the case of a Riemann surface $\Sigma$ of genus $g$ with marked points $x_{1}, \ldots, x_{s}$. We pick rational numbers $\theta_{i}$ between 0 and 1 and let

$$
\Theta_{i} \sim\left(\begin{array}{cc}
e^{\pi i \theta_{i}} & 0 \\
0 & e^{-\pi i \theta_{i}}
\end{array}\right)
$$

be corresponding conjugacy classes in $S U(2)$. We let $\mathscr{M}_{\{\Theta\}}$ be the moduli space of flat connections on $\Sigma-\left\{x_{i}\right\}$ such that the monodromy around $x_{i}$ is in the conjugacy class $\Theta_{i}$. Then the formula (3.1) still makes sense and defines a symplectic structure $\omega$ on $\mathscr{M}_{\{\Theta\}}$. However, the periods of $\omega$ are no longer integers, but are multiples of $1 / m$, where $m$ is the least common denominator of the rational numbers $\theta_{i}$. The latter statement can be proved by exhibiting a line bundle $\mathscr{L}^{\otimes m}$ (which has a natural topological construction [27]) whose first Chern class is represented in de Rham cohomology by $m \omega$. (We call this line bundle $\mathscr{L}^{\otimes m}$ though it is in fact no longer the $m^{\text {th }}$ tensor power of a line bundle $\mathscr{L}$.) The existence of $\mathscr{L}^{\otimes m}$ implies that

$$
[\omega] \in H^{2}\left(\mathscr{M}, \frac{1}{m} \mathbb{Z}\right) .
$$

(This can also be proved by considering a suitable $m$-fold covering of $\Sigma$, as we will do in Sect. 4.7.)

Verlinde's considerations give a formula for $\operatorname{dim} H^{0}\left(\mathscr{M}_{\{\Theta\}}, \mathscr{L}^{\otimes k}\right)$, where $k$ is any integral multiple of $m$ (so that a line bundle $\mathscr{L}^{\otimes k}=\left(\mathscr{L}^{\otimes m}\right)^{\otimes k / m}$ exists). The formula is

$$
\operatorname{dim} H^{0}\left(\mathscr{M}_{\{\Theta\}}, \mathscr{L}^{\otimes k}\right)=\sum_{j=0}^{k} \frac{1}{S_{0, j}^{2 g-2+s}} \cdot \prod_{i=1}^{s} S_{r_{i}, j},
$$

where $\theta_{i}=r_{i} / k$. We wish to extract the large $k$ behavior of this formula. It is convenient to assume first that none of the $\theta_{i}$ is 0 or 1 and thus no $r_{i}$ is 0 or $k$. [The cases that $\theta_{i}=0$ or 1 are special since the corresponding conjugacy classes are in the center of $S U(2)$. If some $\theta_{i}=0$, then the monodromy about $x_{i}$ is trivial and $x_{i}$ can be forgotten. The case that some $\theta_{i}=1$ will be considered later.] With this restriction, the dimension of $\mathscr{M}_{\{\Theta\}}$ is $3 g-3+s$, and we wish to extract the term in (3.16) proportional to $k^{3 g-3+s}$ in the limit of large $k$ with fixed $\theta_{i}=r_{i} / k$. Repeating the derivation of (3.11), we find that in this limit,

$$
\operatorname{dim} H^{0}\left(\mathscr{M}_{\{\Theta\}}, \mathscr{L}^{\otimes k}\right) \sim 2 \cdot \frac{k^{3 g-3+s}}{2^{g-1} \pi^{2 g-2+s}} \sum_{n=1}^{\infty} \frac{\prod_{i=1}^{s} \sin \left(\pi n \theta_{i}\right)}{n^{2 g-2+s}} .
$$

Repeating the use of the Riemann-Roch formula, we learn that the volume of $\mathscr{M}_{\{\Theta\}}$ is

$$
\operatorname{Vol}\left(\mathscr{M}_{\{\Theta\}}\right)=2 \cdot \frac{1}{2^{g-1} \pi^{2 g-2+s}} \sum_{n=1}^{\infty} \frac{\prod_{i=1}^{s} \sin \left(\pi n \theta_{i}\right)}{n^{2 g-2+s}} .
$$

Let us compare this to the formula (2.79) for the Yang-Mills partition function. Setting $\varrho=0$, and recalling that the character of the $n$ dimensional representation of $S U(2)$ is

$$
\chi_{n}(\theta)=\frac{\sin (\pi n \theta)}{\sin (\pi \theta)}
$$


the Yang-Mills partition function is

$$
Z_{\Sigma}(\{\Theta\})=\sum_{n=1}^{\infty} \frac{\prod_{i=1}^{s} \sin \left(\pi n \theta_{i}\right) / \sin \left(\pi \theta_{i}\right)}{n^{2 g-2+s}} .
$$

The relation between the two is supposed to be

$$
\operatorname{Vol}\left(\mathscr{M}_{\{\Theta\}}\right)=2 \cdot Z_{\Sigma}(\{\Theta\}) \cdot e^{(2-2 g-s) \Delta v} \cdot \prod_{i=1}^{s} f\left(\theta_{i}\right),
$$

for some function $f(\theta)$, where we have included the usual factor of 2 coming from the center of $S U(2)$, as well as the normalization ambiguities cited in (2.7) and (2.80). (The latter reflect the fact that no attempt was made in Sect. 2 to pick a particularly felicitous normalization; this will be remedied in Sect. 4.) (3.21) is easy to verify.

Now let us briefly discuss the number theoretic implications of Eq. (3.18). Because of (3.15), $\operatorname{Vol}\left(\mathscr{M}_{\{\Theta\}}\right)$ must be a rational number, whose denominator must divide $m^{r} \cdot r$ !, where $r=\operatorname{dim} \mathscr{M}_{\{\Theta\}} / 2$. Let us identify this rational number, following [9, Chap. 12]. The Hurwitz zeta function is defined for $\operatorname{Re} z>1$ by

$$
\zeta(z, a)=\sum_{n=0}^{\infty} \frac{1}{(n+a)^{z}},
$$

and is then continued holomorphically throughout the complex $z$ plane, except for a pole at $z=1$. The periodic zeta function is defined by

$$
F(x, z)=\sum_{n=1}^{\infty} \frac{e^{2 \pi i n x}}{n z} .
$$

A formula of Hurwitz (Theorem 12.6 in [9]) asserts that for $0<a \leqq 1$ and $\operatorname{Re} z>1$,

$$
\zeta(1-z, a)=\frac{\Gamma(z)}{(2 \pi)^{z}} \cdot\left(e^{-i \pi z / 2} F(a, z)+e^{\pi i z / 2} F(-a, z)\right) .
$$

These functions are closely related to Dirichlet $L$-functions. Let us now, for illustrative purposes, consider (3.18) for a surface of genus $g$ with one puncture. We set $a=\theta / 2, z=2 g-1$, and by comparing (3.18) and (3.24), we find

$$
\operatorname{Vol}\left(\mathscr{M}_{\{\Theta\}}\right)=\frac{2^{g}(-1)^{g+1}}{\Gamma(2 g-1)} \cdot \zeta(2-2 g, \theta / 2) .
$$

Thus, the known facts about the volume of $\mathscr{M}_{\{\Theta\}}$ amount to the statement that for positive integer $g$ and rational $\theta$ between 0 and $1, \zeta(2-2 g, \theta / 2)$ is a rational number, with an explicit bound on the denominator. This is in accord with the explicit evaluation of $\zeta(z, a)$ for negative integral $z$ (Theorem 12.12 of [9]). The more general case of (3.18) with more than one puncture can be treated similarly, expanding the product of sines on the right-hand side of (3.18) as a sum of sines or cosines and expressing the volume as a sum of values of $\zeta(z, a)$.

\subsection{Corresponding Formulas for $\mathrm{SO}(3)$}

While there is only one topological type of an $S U(2)$ bundle on a two dimensional closed surface $\Sigma$, there are two topological types of $S O(3)$ bundles, corresponding 
to $w_{2}=0$ and $w_{2} \neq 0$, respectively. The moduli space $\mathcal{N}$ of flat $S O(3)$ bundles on $\Sigma$ thus has two components, which we will call $\mathscr{N}^{\prime}$ and $\mathscr{N}^{\prime \prime}$, corresponding to flat connections on bundles of these two types. $\mathscr{N}^{\prime}$ and $\mathscr{N}^{\prime \prime}$ are both symplectic manifolds, with symplectic structures deduced from (3.1). We recall from the beginning of Sect. 2 that we use the same quadratic form Tr on the $S U(2)$ and $S O(3)$ Lie algebras, which are in fact naturally isomorphic. The formula (3.1) thus makes sense for $S O(3)$, though it defines a symplectic structure whose periods are in general half integral. That symplectic structure gives a definition of volume forms on both components of $\mathscr{N}$.

We will use the Verlinde formula to determine the volumes of both $\mathscr{N}^{\prime}$ and $\mathscr{N}^{\prime \prime}$. For $\mathscr{N}^{\prime}$ this is easy. The natural transformation from an $S U(2)$ bundle $E$ to the adjoint bundle $\operatorname{ad}(E)$ gives a map from $\mathscr{M}$ to $\mathscr{N}^{\prime}$. This map is locally an isomorphism; globally it is an unramified cover of degree $2^{2 g}$. This factor arises because if $\eta$ is a flat complex line bundle of order 2, then the flat $S U(2)$ bundle $E^{\prime}=E \otimes \eta$ has $\operatorname{ad}\left(E^{\prime}\right) \cong \operatorname{ad}(E)$; there are $2^{2 g}$ choices of $\eta$. We thus have

$$
\operatorname{Vol}\left(\mathscr{N}^{\prime}\right)=2^{-2 g} \operatorname{Vol}(\mathscr{M})=2^{1-2 g} \cdot \frac{1}{\left(2 \pi^{2}\right)^{g-1}} \sum_{n=1}^{\infty} n^{-(2 g-2)} .
$$

To determine the volume of $\mathcal{N}^{\prime \prime}$ is less trivial. To this end, we return to our study of $S U(2)$, and consider the previously neglected case that one of the $\theta$ 's is 1 , so that the corresponding $r$ is $k$, and the monodromy around the corresponding puncture is the central element -1 of $S U(2)$. In fact, let us consider the case in which there is just one puncture $x$, with this monodromy. Let $\mathscr{M}(-1)$ be the corresponding moduli space. $\mathscr{M}(-1)$ has dimension $3 g-3$ (just like $\mathscr{M}$ ) so its volume is to be computed by extracting the coefficient of $k^{3 g-3}$ in the asymptotic behavior of (3.16). Since $S_{j i} / S_{0 i}=(-1)^{i}$ for $j=k$ (as one sees from the explicit formula for $S$ ), the steps leading to (3.11) are readily repeated, with the result

$$
\operatorname{Vol}(\mathscr{M}(-1))=2 \cdot \frac{1}{\left(2 \pi^{2}\right)^{g-1}} \cdot \sum_{n=1}^{\infty}(-1)^{n+1} n^{-(2 g-2)} .
$$

A flat $S U(2)$ connection $A$ on $\Sigma-\{x\}$, with monodromy -1 about $x$, determines a flat $S O(3)$ connection $A^{\prime}$ on an $S O(3)$ bundle with $w_{2} \neq 0$ (simply because the monodromy -1 is undetectable at the $S O(3)$ level). This gives a natural map $\mathscr{M}(-1) \rightarrow \mathscr{N}^{\prime \prime}$, which is an isomorphism locally and which globally is a cover of order $2^{2 g}$ [again, because twisting by a flat line bundle of order two is undetectable at the $S O(3)$ level]. Hence we have

$$
\operatorname{Vol}\left(\mathscr{N}^{\prime \prime}\right)=2^{-2 g} \operatorname{Vol}(\mathscr{M}(-1))=2^{1-2 g} \cdot \frac{1}{\left(2 \pi^{2}\right)^{g-1}} \sum_{n=1}^{\infty}(-1)^{n+1} n^{-(2 g-2)}
$$

Adding (3.26) to (3.28), the combined volume of the $S O(3)$ moduli space is

$$
\operatorname{Vol}(\mathscr{N})=\operatorname{Vol}\left(\mathscr{N}^{\prime}\right)+\operatorname{Vol}\left(\mathscr{N}^{\prime \prime}\right)=\frac{1}{\left(8 \pi^{2}\right)^{g-1}} \sum_{n=1,3,5, \ldots} \frac{1}{n^{2 g-2}}
$$

Since the dimensions of $S O(3)$ representations are the odd integers $1,3,5, \ldots$, the Yang-Mills formula (2.51) gives in this case

$$
Z_{\Sigma}=\sum_{n=1,3,5, \ldots} n^{-(2 g-2)} .
$$

We see thus the expected relation

$$
\operatorname{Vol}(\mathscr{N})=Z_{\Sigma} \cdot e^{\Delta v \cdot(2 g-2)},
$$


with a suitable value of $\Delta v$. No factor of 2 appears here, since the center of $S O(3)$ is trivial.

Notice that, although in Sect. 2 we never addressed the question of the classification of $G$ bundles on $\Sigma$, the computation carried out there automatically gave the correct answer summed over all topological types of $G$ bundles. Indeed the sewing rules used in Sect. 2 would not be compatible with any restriction on the topological type of the bundle.

Obviously we could go on to combine the above constructions and consider $S O(3)$ flat connections on surfaces with punctures. There are no essentially new issues, so we will forgo this step.

\section{Evaluation of the Torsion}

\subsection{Preliminaries}

In this section, we will use the purely combinatorial definition of the Reidemeister torsion of a flat connection to give a direct computation of the volumes of moduli space of representations of fundamental groups of two dimensional surfaces. (The combinatorial torsion is known $[29,28]$ to be equivalent to the Ray-Singer analytic torsion, which arose more naturally in Sect. 2 . The equivalence is actually elementary for orientable even dimensional manifolds since both are "trivial.") The computation will proceed by imitating some of the steps in Sects. 2.3-4 in the context of torsion. However, in contrast to that discussion, where an arbitrary lattice regularization made it impossible to nail down the renormalization constant $\Delta v$ of Eq. (2.9), here we will be precise with all of the constants. Hence, we must at the outset state a few conventions.

We will restrict ourselves to compact, semi-simple, connected (but not necessarily simply connected) gauge groups $G$. In what follows, $(a, b)$, which we will denote for convenience as - Tr $a b$, denotes an arbitrary invariant quadratic form on the Lie algebra $\mathscr{G}$ of $G$, which is used consistently in defining all Haar measures, volumes on chain groups, etc. In case $G$ is simple, there is a particular choice, described at the beginning of Sect. 2, that is convenient for comparing to formulas of conformal field theory. For $G=S U(N)$, this choice is $(a, b)=-\operatorname{Tr} a b$, where $\operatorname{Tr}$ is the trace in the $N$ dimensional representation. For instance, for $G=S U(2)$, if we write an element of $G$ as

$$
U=\left(\begin{array}{rr}
a & b \\
-\bar{b} & \bar{a}
\end{array}\right),
$$

with $\bar{a} a+\bar{b} b=1$, then the metric is

$$
d s^{2}=2(d a d \bar{a}+d b d \bar{b}) .
$$

This is twice the usual metric on the $S U(2)$ manifold. We emphasize, though, that the formulas of this section are valid with any invariant metric on $\mathscr{G}$, as long as it is consistently used throughout.

An invariant metric on $\mathscr{G}$ determines a left and right invariant measure on $G$, namely the Riemannian volume element $\mu$. We will refer to this measure as Haar measure on G. (In Sect. 2.3, where we carried out a less precise calculation, we simply normalized Haar measure so that the volume of $G$ was one.) Computing the 


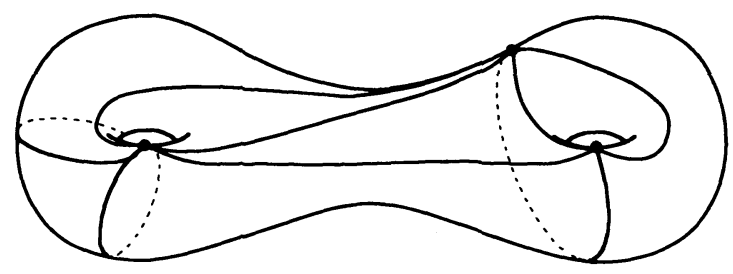

Fig. 10. A cell decomposition determined by a covering of $\Sigma$ by polygons

volume of the $S U(2)$ manifold with the metric (4.2), we find $\operatorname{Vol}(S U(2))=2^{5 / 2} \pi^{2}$, $2^{3 / 2}$ times larger that the usual expression because of the 2 in (4.2). The volume of $S O(3)$ is half this or $2^{3 / 2} \pi^{2}$.

We also will be interested in the maximal torus $T$ and in the moduli space $L$ of conjugacy classes in $G$; we recall that for compact, connected $G$, the latter is $L=T / W$, with $W$ the Weyl group. The metric on $\mathscr{G}$ restricts to a metric on the Lie algebra $\mathscr{T}$ of $T$. The latter determines a Riemannian measure on $T$, which we will call the Haar measure $v_{0}$. Since (away from the singularities in the Weyl action, which occur on a set of positive codimension), $L$ is locally modeled on $T$, the Haar measure on $T$ determines a measure on $L$ which we will also call $v_{0}$. (Of course, the volume of $L$ in the $v_{0}$ measure is less than the $v_{0}$ volume of $T$ by a factor of \# $W$, the order of the Weyl group.)

There is a second important measure on $L$ which arises as follows. The association of a group element with its conjugacy class gives a natural map $u: G$ $\rightarrow L$. We set $v=u_{*}(\mu)$. The precise comparison between $v$ and $v_{0}$ will be important later.

We will take advantage of the following simplifications. First of all, since we consider volumes to be positive by definition, and since we will always be multiplying rather than adding torsions, we do not need to worry about the overall sign of the torsion. Thus, like much of the literature, our description of the torsion will be valid only up to sign. Also, as our goal is only to compute volumes, exceptional sets of positive codimension will not contribute, and will frequently be discarded. (Among other things, it is for this reason that we need not be concerned about singularities of $\mathscr{M}$.)

The computation that we will carry out is fairly short and elementary. The length of this section comes from the attempt to be self-contained in explaining the combinatorial torsion and from the fact that we work out several cases in detail. Further background about combinatorial torsion can be found in [11-13].

\subsection{Definition of the Torsion}

To begin with, consider a particular flat connection $A$ on a $G$ bundle $E$ over a surface $\Sigma$. Let $\operatorname{ad}(E)$ denote the corresponding adjoint bundle. If $A$ is irreducible, then $H_{0}(\Sigma, \operatorname{ad}(E))=H_{2}(\Sigma, \operatorname{ad}(E))=0$, and $H_{1}(\Sigma, \operatorname{ad}(E))=\left.T^{*} \mathscr{M}\right|_{A}$ is the cotangent space to $\mathscr{M}$ at $A$. This will play an important role, so we want to recall the combinatorial definition of the homology of $\Sigma$ with values in ad $(E)$. We consider a cell decomposition $X$ of $\Sigma$ of the same type that we used in lattice gauge theory in Sect. 2.3, consisting of a covering of $\Sigma$ by polygons (Fig. 10). The polygons are twocells, and their edges and vertices are one-cells and zero-cells. If the cell 


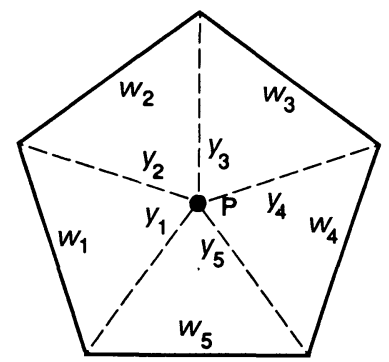

Fig. 11. Subdivision of an $n$-gon by adding one-cells $y_{i}$ (indicated with dotted lines)

decomposition is fine enough, the flat connection $A$ is trivial on any given cell, and then for each cell $w$ we define a vector space $V_{w}$ consisting of parallel sections of $\operatorname{ad}(E)$ over $w$. (It will be convenient later to allow less fine cell decompositions in which some one or two cell $w$ may be large enough that $\left.A\right|_{w}$ is not trivial; then one defines $V_{w}$ to consist of parallel sections over the interior of $w$. The definition of the boundary operator $\partial$ that we are about to give then needs a slight refinement.) Metrics on the $V_{w}$ 's are defined by saying that the inner product of parallel sections $s$ and $s^{\prime}$ is the constant $-\operatorname{Tr} s s^{\prime}$.

Given a real vector space $A$, we let $\operatorname{det} A$ denote the top exterior power of $A$. By a measure on $A$ we mean a nonzero linear functional on $\operatorname{det} A$. A metric on $A$ determines (up to a sign, which as noted above we will not keep track of) such a measure, namely the functional that maps $x_{1} \wedge x_{2} \ldots \wedge x_{n}$ to 1 , where the $x_{i}$ are an orthonormal basis of $A$. So the metrics on the $V_{w}$ endow those vector spaces with measures.

For $q=0,1,2$, let $C_{q}=\bigoplus_{w} V_{w}$, where $w$ runs over all $q$-cells. If the boundary of $w$ is a union of $q-1$-cells $y_{i}$, then one defines a map $\partial: V_{w} \rightarrow \oplus V_{y_{i}}$ by $\partial s=\left.\bigoplus_{i} s\right|_{y_{i}}$. This gives a chain complex $C_{\bullet}$,

$$
0 \rightarrow C_{2} \stackrel{\partial}{\longrightarrow} C_{1} \stackrel{\partial}{\longrightarrow} C_{0} \rightarrow 0 .
$$

The homology of this chain complex is $H_{*}(\Sigma, \operatorname{ad}(E))$.

It is instructive to recall the proof that up to canonical isomorphism, $H_{*}(\Sigma, \operatorname{ad}(E))$ is independent of the choice of cell decomposition. The main step is to verify invariance under subdivision. As a typical example, let $w$ be a two-cell (which we take to be an $n$-gon) which is to be subdivided by adding an extra vertex $p$ (Fig. 11). In the refined cell decomposition $\hat{X}, w$ is the union of $n$ two-cells $w_{1}, \ldots, w_{n} ; n$ one-cells $y_{1}, \ldots, y_{n}$ have been added. The refined cell decomposition $\hat{X}$ gives a new chain complex $\hat{C}_{\bullet}$.

A natural injective chain map $i: C_{q} \rightarrow \hat{C}_{q}$ is defined as follows. Any cell $w^{\prime}$ in $X$ is subdivided into a union of cells $v_{j}$ in $\hat{X}$ (for $w^{\prime} \neq w$, there is just one $v$ ): one maps $V_{w^{\prime}} \subset C_{\bullet}$ to $\bigoplus_{j} V_{v_{j}} \subset \hat{C} \bullet$ by $i(s)=\left.\bigoplus_{j} s\right|_{v_{j}}$. As this map is injective, we get a short exact sequence of chain complexes,

$$
O \rightarrow C_{\bullet} \stackrel{i}{\longrightarrow} \hat{C}_{\bullet} \rightarrow C_{\bullet}^{\prime} \rightarrow 0,
$$

where $C_{\bullet}^{\prime}$ is the quotient $\hat{C}_{\bullet} / C_{\bullet}$. The associated long exact sequence of homology,

$$
0 \rightarrow \mathrm{H}_{2}(C) \rightarrow \mathrm{H}_{2}(\widehat{C}) \rightarrow \mathrm{H}_{2}\left(C^{\prime}\right) \rightarrow \ldots \rightarrow H_{0}\left(C^{\prime}\right) \rightarrow 0
$$


shows that $H_{*}(C)$ is canonically isomorphic to $H_{*}(\hat{C})$ - as we wished to prove provided $H_{*}\left(C^{\prime}\right)=0$.

To compute $H_{*}\left(C^{\prime}\right)$, we note that $E$ can be parallelized over $w$ (or at least its interior) and hence will play no role in the computation. We may as well take the coefficient group to be $\mathbb{R}$, and in that case, for any cell $y$, we let [y] denote the basis of the one dimensional vector space $V_{y}$ corresponding to the section 1 . The quotient $C_{\bullet}^{\prime}=\hat{C}_{\bullet} / C_{\bullet}$ comes from cells added as a result of the subdivision of $X$. So $C_{0}^{\prime}$ has one generator $[p], C_{1}^{\prime}$ has $n$ generators $\left[y_{1}\right], \ldots,\left[y_{n}\right]$, and $C_{2}^{\prime}$ has $n$ generators $\left[w_{1}\right], \ldots,\left[w_{n}\right]$ with one relation

$$
\left[w_{1}\right]+\left[w_{2}\right]+\ldots+\left[w_{n}\right]=0 .
$$

The boundary operator is

$$
\begin{aligned}
\partial\left[w_{i}\right] & =\left[y_{i+1}\right]-\left[y_{i}\right], \\
\partial\left[y_{i}\right] & =[p]
\end{aligned}
$$

(where we identify $\left[y_{i+n}\right]=\left[y_{i}\right]$ ). It is easily seen that this complex is acyclic, as we wished to show.

Now we wish to introduce the torsion, which is defined, in an appropriate sense, for any chain complex of vector spaces with measures. We will follow [13]. Let

$$
F_{\bullet}: 0 \rightarrow F_{n} \stackrel{\partial}{\longrightarrow} F_{n-1} \stackrel{\partial}{\longrightarrow} \ldots \stackrel{\partial}{\longrightarrow} F_{1} \stackrel{\partial}{\longrightarrow} F_{0} \rightarrow 0
$$

be a chain complex of finite dimensional vector spaces, with volumes $\alpha_{k} \in\left(\operatorname{det} F_{k}\right)^{-1}$. (This can also be relaxed slightly, if one has only the products of the $\alpha_{k}$ that appear later rather than individual $\alpha_{k}$ 's.) We first consider the case that $F_{\bullet}$ is acyclic. If $k_{j}=\operatorname{dim} \operatorname{im} \partial: F_{j} \rightarrow F_{j-1}$, pick $s_{j} \in \wedge{ }^{k_{j}} F_{j}$ such that $\partial s_{j} \neq 0$. The expression

$$
u=\bigotimes_{j=0}^{n}\left(\partial s_{j+1} \wedge s_{j}\right)^{(-1)^{j}} \in \bigotimes_{j=0}^{n}\left(\operatorname{det} F_{j}\right)^{(-1)^{j}}
$$

is independent of the choices of the $s_{j}$, so we can define the torsion as

$$
\tau\left(F_{\bullet}\right)=u \bigotimes_{k=0}^{n} \alpha_{k}^{(-1)^{k}}
$$

More generally, any chain complex $F_{\bullet}$ can be split (unnaturally) as $F_{\bullet}=F_{\bullet}^{\prime} \oplus F_{\bullet}^{\prime \prime}$, where $F_{\bullet}^{\prime}$ is acyclic and $F_{\bullet}^{\prime \prime}$ has zero differential. (Hence $F_{n}^{\prime \prime} \cong H_{n}\left(F_{\bullet}\right)$ are the homology groups of $F_{\bullet}$.) Now letting $k_{j}=\operatorname{dim} \operatorname{im} \partial: F_{j}^{\prime} \rightarrow F_{j-1}^{\prime}$ and picking $s_{j} \in \wedge{ }^{k_{j}} F_{k_{j}}^{\prime}$ such that $\partial s_{j} \neq 0$, we define

$$
u=\bigotimes_{j=0}^{k}\left(\partial s_{j+1} \wedge s_{j}\right)^{(-1)^{j}} \in \bigotimes_{j=0}^{n}\left(\operatorname{det} F_{j}^{\prime}\right)^{(-1)^{j}}
$$

and

$$
\tau\left(F_{\bullet}\right)=u \bigotimes_{j=0}^{n}\left(\alpha_{j}\right)^{(-1)^{j}} \in \bigotimes_{j=0}^{n}\left(\operatorname{det} F_{j}^{\prime \prime}\right)^{(-1)^{j+1}}=\bigotimes_{j=0}^{n}\left(\operatorname{det} H_{j}(F)\right)^{(-1)^{j+1}}
$$
Thus $\tau$ lies in the dual of the one dimensional vector space $\bigotimes_{i=0}^{n}\left(\operatorname{det} H_{i}\left(F_{\bullet}\right)\right)^{(-1)^{i}}$
which is usually called $\operatorname{det} H_{*}\left(F_{\bullet}\right)$.

In particular, going back to the chain complex $C_{\bullet}$ that computes $H_{*}(\Sigma, \operatorname{ad}(E))$, we can define the torsion $\tau\left(C_{\bullet}\right)$, which is a vector in $\left(\operatorname{det} H_{0}(\Sigma, \operatorname{ad}(E))\right)^{-1} \otimes \operatorname{det} H_{1}(\Sigma, \operatorname{ad}(E)) \otimes\left(\operatorname{det} H_{2}(\Sigma, \operatorname{ad}(E))\right)^{-1}$. For an irreducible 
flat connection, $H_{0}(\Sigma, \operatorname{ad}(E))=H_{2}(\Sigma, \operatorname{ad}(E))=0$, so $\tau\left(C_{\bullet}\right)$ is defined as a vector in $\operatorname{det} H_{1}(\Sigma, \operatorname{ad}(E))$.

We want to verify first that $\tau\left(C_{0}\right)$ depends only on the connection $A$ and is independent of the choice of cell decomposition $X$. The main step is to show invariance under subdivision, so we return to the short exact sequence (4.4), which in more detail is a commuting diagram

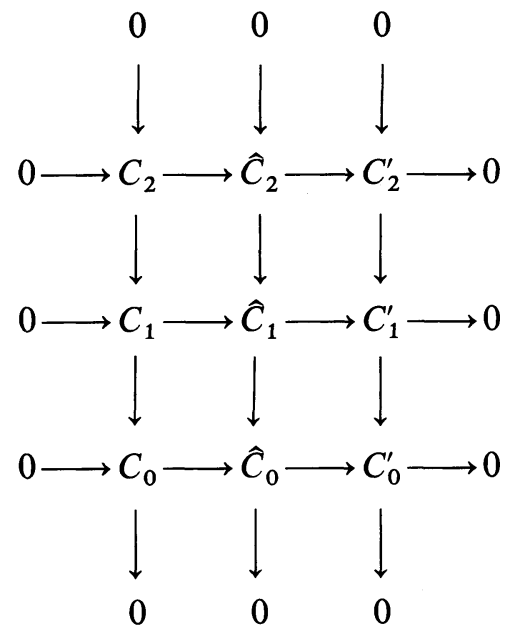

whose columns are the chain complexes $C_{\bullet}, \hat{C}_{0}$, and $C_{0}^{\prime}$. Each chain group here has a volume. The rows are acyclic, and it is obvious from the definitions that the torsion of each row is 1 . A general proposition proved by linear algebra (Proposition (21) of [12]) then asserts that the alternating product of the torsions of the columns is also 1 , that is,

$$
\tau\left(C_{\bullet}\right) \tau\left(\hat{C}_{\bullet}\right)^{-1} \tau\left(C_{\bullet}^{\prime}\right)=1 \text {. }
$$

To obtain the desired result $\tau\left(C_{\bullet}\right)=\tau\left(\hat{C}_{\bullet}\right)$ showing the invariance of the torsion under subdivision, we thus need to know that $\tau\left(C_{\bullet}^{\prime}\right)=1$. This can be routinely verified from the explicit description of $C^{\prime}$ in (4.7).

We will describe the fact that the rows in (4.13) have torsion 1 by saying that the exact sequence (4.4) is volume-exact. Equation (4.14) is then the assertion that in an exact sequence of complexes which is also volume-exact, the alternating product of the torsions is one.

Consider now the moduli space $\mathscr{M}$ of flat $G$-valued connections on $\Sigma$. The association to a connection $A$ of the corresponding $H_{1}(\Sigma, \operatorname{ad}(E))$ defines a vector bundle over $\mathscr{M}$ which is none other than the cotangent bundle $T^{*} \mathscr{M}$. The torsion $\tau(A)$ is a section of $\operatorname{det}\left(T^{*} \mathscr{M}\right)$, or in other words a differential form of top degree on $\mathscr{M}$. Actually, since we have not paid attention to signs, it is really only the absolute value of $\tau$ that is well-defined; this is properly regarded as a measure on $\mathscr{M}$. In any case, we have achieved our goal of giving a topological construction of a measure on $\mathscr{M}$, without assuming orientability of $\Sigma$.

We define the volume of $\mathscr{M}$ to be

$$
\operatorname{Vol}(\mathscr{M})=\frac{1}{(2 \pi)^{\operatorname{dim} \mathscr{M}}} \int_{\mathscr{M}} \tau,
$$


where $\operatorname{dim} \mathscr{M}$ is the (real) dimension of $\mathscr{M}$. The reason for the power of $2 \pi$ is to agree, as we will see shortly, with the normalization that is natural from the symplectic point of view and was used in Sect. 3.

Comparison to the Symplectic Volume. We now wish, for $\Sigma$ orientable, to compare the measure on $\mathscr{M}$ given by the torsion to that which can be constructed using the symplectic structure on $\mathscr{M}$.

To begin with, we consider, algebraically, what we will call a symplectic complex. This is a complex

$$
0 \rightarrow C_{n} \stackrel{\partial}{\longrightarrow} C_{n-1} \stackrel{\partial}{\longrightarrow} \ldots \stackrel{\partial}{\longrightarrow} C_{0} \rightarrow 0
$$

with $n \equiv 2 \bmod 4$, and with non-degenerate pairings $\lambda_{p, n-p}: C_{p} \otimes C_{n-p} \rightarrow \mathbb{R}$ (collectively we will call them $\lambda$ ) which are skew-symmetric in the sense that $\lambda_{p, n-p}(a, b)$ $=(-1)^{p} \lambda_{n-p, p}(b, a)$, and are compatible with $\partial$ in the sense that $\lambda_{p, n-p}(\partial x, b)$ $=(-1)^{p+1} \lambda_{p, n-p}(x, \partial b)$.

The compatibility of $\lambda$ with $\partial$ means that the $\lambda$ 's induce pairings

$$
\bar{\lambda}_{p, n-p}: H_{p}(C) \otimes H_{n-p}(C) \rightarrow \mathbb{R},
$$

which by linear algebra are seen to be nondegenerate. Pick a lift of $H_{p}(C)$ to $C_{p}$, and let $C_{p}^{\prime \prime}$ be the image. If $C_{p}^{\prime}$ denotes the kernel of $\lambda_{p, n-p}: C_{p} \otimes C_{n-p}^{\prime \prime} \rightarrow \mathbb{R}$, then we get a splitting $C_{\bullet}=C_{\bullet}^{\prime} \oplus C_{\bullet}^{\prime \prime}$, which is compatible with $\lambda$ in the sense that for $a \in C_{p}^{\prime}$, $b \in C_{n-p}^{\prime \prime}, \lambda_{p, n-p}(a, b)=0 . C^{\prime}$ is an acyclic chain complex, and $C_{\bullet}^{\prime \prime}$ is a complex with zero boundary operator.

Given $\lambda_{p, n-p}: C_{p} \otimes C_{n-p} \rightarrow \mathbb{R}$, there is an induced map $\operatorname{det} C_{p} \otimes \operatorname{det} C_{n-p} \rightarrow \mathbb{R}$ which we will call $\operatorname{det} \lambda_{p, n-p}$. It is an element of $\left(\operatorname{det} C_{p} \otimes \operatorname{det} C_{n-p}\right)^{-1}$. For $p=n / 2$, given an orientation of $\operatorname{det} C_{n / 2}, \lambda_{n / 2, n / 2} \in \operatorname{det} C_{n / 2}^{\otimes 2}$ has a natural square root which we call the Pfaffian, Pfaff $\lambda_{n / 2, n / 2}$. It is an element of $\left(\operatorname{det} C_{n / 2}\right)^{-1}$. (Actually, since we are not keeping track of the overall sign of the torsion, we do not need the canonical square root of $\operatorname{det} \lambda_{n / 2, n / 2}$.) A system of measures $\alpha_{p} \in\left(\operatorname{det} C_{p}\right)^{-1}$ will be called compatible with the $\lambda_{p, n-p}$ if $\alpha_{p} \otimes \alpha_{n-p}=\operatorname{det} \lambda_{p, n-p}$ for all $p$, and $\alpha_{n / 2}$ $=\operatorname{Pfaff} \lambda_{n / 2, n / 2}$.

We now claim that the torsion of a symplectic complex $\left(C_{0}, \lambda\right)$ computed using a compatible set of measures is "trivial" in the sense that

$$
\tau\left(C_{0}\right)=\bigotimes_{p=0}^{n / 2-1}\left(\operatorname{det} \bar{\lambda}_{p, n-p}\right)^{(-1)^{p}} \otimes\left(\text { Pfaff } \bar{\lambda}_{n / 2, n / 2}\right)^{(-1)^{n / 2}} .
$$

In view of the splitting $C_{\bullet}=C_{\bullet}^{\prime} \oplus C_{\bullet}^{\prime \prime}$, it is enough to check the cases that $C_{\bullet}$ is acyclic or has zero boundary operator. If the boundary operator is zero, then (4.18) is the definition of the torsion. If $C_{\bullet}$ is acyclic, then the claim is that $\tau\left(C_{0}\right)=1$. Given that $\lambda$ can be regarded as an identification of $C_{\bullet}$ with the dual complex $\widetilde{C}_{\bullet}=\operatorname{Hom}\left(C_{\bullet}, \mathbb{R}\right)$, this is a special case of the relation between the torsion of a complex and that of the dual complex. See for instance Proposition (14) of [12].

We now return to our problem of comparing the measure on $\mathscr{M}$ given by the torsion to the measure determined by the symplectic structure. The symplectic structure is equivalent to a nondegenerate skew map $H_{1}(\Sigma, \operatorname{ad}(E)) \otimes H_{1}(\Sigma, \operatorname{ad}(E))$ $\rightarrow \mathbb{R}$, and we wish to see this map combinatorially.

For every cell decomposition $X$ of $\Sigma$ by polygons, there is a dual cell decomposition $X^{\prime}$ with $q$ cells replaced by $2-q$ cells (Fig. 12). $X$ and $X^{\prime}$ correspond to chain complexes $C_{\bullet}$ and $C_{\bullet}^{\prime}$, each with metrics and measures on the chain 


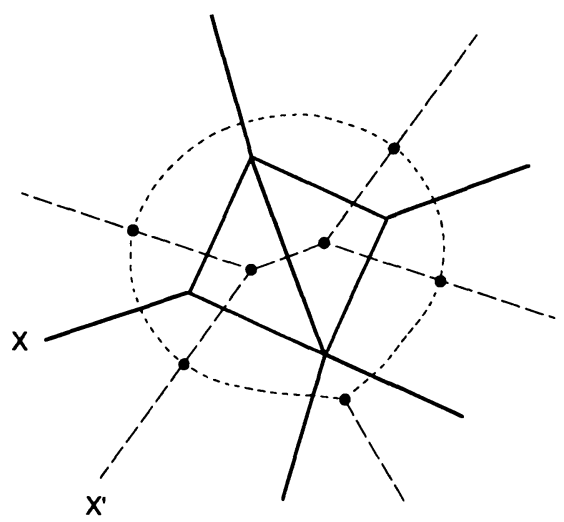

Fig. 12. A cell division $X$ and its dual $X^{\prime}$

groups. Invariance of the torsion under subdivision means that $\tau\left(C_{\bullet}\right)=\tau\left(C_{\bullet}^{\prime}\right)$. We will study the complex $D_{\bullet}=C_{\bullet} \oplus C^{\prime} \bullet$ with the product measures; its torsion is

$$
\tau\left(D_{\bullet}\right)=\tau\left(C_{\bullet}\right) \cdot \tau\left(C_{\bullet}^{\prime}\right)=\tau\left(C_{\bullet}\right)^{2} .
$$

Natural pairings $C_{p} \otimes C_{2-p}^{\prime} \rightarrow \mathbb{R}$ can be defined essentially by counting intersections of cells. Let $w$ and $w^{\prime}$ be a $p$-cell and a $2-p$-cell in $C_{p}$ and $C_{2-p}^{\prime}$, respectively. The corresponding spaces $V_{w} \subset C_{p}$ and $V_{w^{\prime}} \subset C_{2-p}^{\prime}$ consist of parallel sections of $\operatorname{ad}(E)$ over $w$ and $w^{\prime}$, respectively. Given sections $s$ and $s^{\prime}$ defining elements of $V_{w}$ and $V_{w^{\prime}}$, we let

$$
\lambda\left(s, s^{\prime}\right)=-\operatorname{Tr}\left(s s^{\prime}\right) \cdot \sum_{P \in w \cap w^{\prime}}( \pm 1),
$$

where the sign associated with $P \in w \cap w^{\prime}$ is the usual algebraic intersection number. (We have defined $X$ and $X^{\prime}$ so that $w \cap w^{\prime}$ contains at most one point.) By defining $\lambda\left(s^{\prime}, s\right)=(-1)^{p} \lambda\left(s, s^{\prime}\right)$, and defining $\lambda$ to vanish on $C_{p} \otimes C_{2-p}$ and on $C_{p}^{\prime} \otimes C_{2-p}^{\prime}$, we extend the $\lambda$ 's to symplectic pairings $\lambda_{p, 2-p}: D_{p} \otimes D_{2-p} \rightarrow \mathbb{R}$. These give $D_{\bullet}$ the structure of a symplectic complex, and moreover, the $\lambda$ 's are compatible with the natural measures on $D_{\mathbf{c}}$. Hence we get

$$
\tau\left(D_{\bullet}\right)=\operatorname{det} \bar{\lambda}_{0,2} \otimes\left(\text { Pfaff } \bar{\lambda}_{1,1}\right)^{-1} .
$$

For an irreducible flat connection, $H_{0}(\Sigma, \operatorname{ad}(E))=H_{2}(\Sigma, \operatorname{ad}(E))=0$, so

$$
\tau\left(D_{\bullet}\right)=\left(\operatorname{Pfaff} \bar{\lambda}_{1,1}\right)^{-1} \text {. }
$$
form,

Now, in terms of the decomposition $\left.H_{1}\left(D_{\bullet}\right) \cong H_{1}\left(C_{\bullet}\right) \oplus H_{1}\left(C_{0}^{\prime}\right)\right), \bar{\lambda}_{1,1}$ has a block

$$
\bar{\lambda}_{1,1}=\left(\begin{array}{rr}
0 & \varrho \\
-\varrho & 0
\end{array}\right),
$$

where, as $H_{1}\left(C_{\bullet}\right)$ and $H_{1}\left(C_{\bullet}^{\prime}\right)$ are both canonically isomorphic to $H_{1}(\Sigma, \operatorname{ad}(E)), \varrho$ can be regarded as a skew form $\Lambda^{2} H_{1}(\Sigma, \operatorname{ad}(E)) \rightarrow \mathbb{R} .^{5}$ We have Pfaff $\bar{\lambda}_{1,1}=(\text { Pfaff } \varrho)^{2}$. As also $\tau\left(D_{\bullet}\right)=\tau\left(C_{\bullet}\right)^{2}$, we get

$$
\tau\left(C_{\bullet}\right)=(\operatorname{Pfaff} \varrho)^{-1}=\operatorname{Pfaff}\left(\varrho^{-1}\right),
$$

${ }_{5}$ Our argument here does not prove the skewness, but this is standard 
(a)

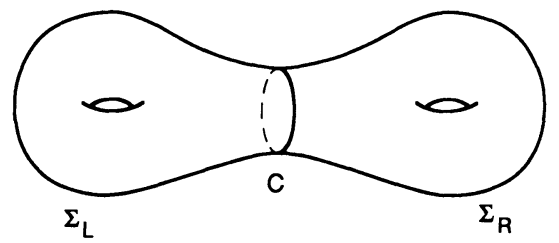

(b)

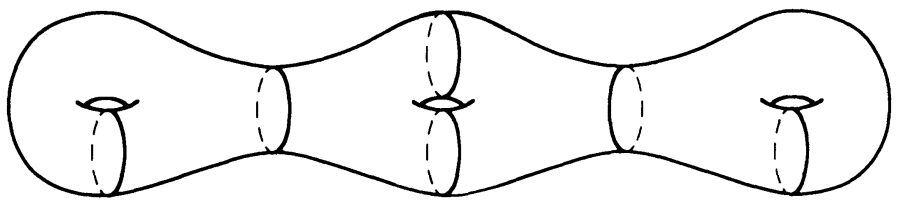

Fig. 13a, b. Cutting a surface $\Sigma$ on a circle $S$ to decompose it into pieces $\Sigma_{L}$ and $\Sigma_{R}$, with corresponding cell decompositions a. Decomposing an oriented surface $\Sigma$ of genus $g$ into $2 g-2$ three holed spheres b

where $\varrho^{-1}: \Lambda^{2} H^{1}(\Sigma, \operatorname{ad}(E)) \rightarrow \mathbb{R}$ is the inverse to $\varrho$. (If $\varrho$ is given by a matrix $\varrho_{i j}$ in some basis on $H_{1}$, then $\varrho^{-1}$ is given in the dual basis on $H^{1}$ by the inverse matrix.)

Now, $H^{1}(\Sigma, \operatorname{ad}(E))$ is the tangent space to the moduli space $\mathscr{M}$ of flat connections, and hence $\varrho^{-1}: \Lambda^{2} H^{1}(\Sigma, \operatorname{ad}(E)) \rightarrow \mathbb{R}$ is a two form on $\mathscr{M}$. If $\mathscr{M}$ has $\operatorname{dimension} \operatorname{dim} \mathscr{M}=2 r$, then $\operatorname{Pfaff} \varrho^{-1}$ is the top form

$$
\operatorname{Pfaff} \varrho^{-1}=\frac{\left(\varrho^{-1}\right)^{r}}{r !} .
$$

Actually, because we have defined $\varrho$ and $\varrho^{-1}$ by intersection of cycles, $\varrho^{-1}$ may be described explicitly in the de Rham model by saying that to $\alpha, \beta \in H^{1}(\Sigma, \operatorname{ad}(E)), \varrho^{-1}$ assigns the value

$$
\varrho^{-1}(\alpha, \beta)=\int_{\Sigma} \operatorname{Tr}(\alpha \wedge \beta)
$$

Comparing this to the definition of the symplectic form $\omega$, which was

$$
\omega(\alpha, \beta)=\frac{1}{4 \pi^{2}} \int_{\Sigma} \operatorname{Tr}(\alpha \wedge \beta),
$$

we see $\varrho^{-1}=4 \pi^{2} \omega$. Hence, with (4.24) and (4.25), we see that the torsion volume of $\mathscr{M}$ as defined in (4.15) indeed agrees with the symplectic volume:

$$
\operatorname{Vol}(\mathscr{M})=\frac{1}{(2 \pi)^{\operatorname{dim} \mathscr{M}}} \int_{\mathscr{M}} \tau=\int_{\mathscr{M}} \frac{\omega^{r}}{r !} .
$$

Cutting Formula. In the quantum field theory computation of Sects. 2.3-4, one important ingredient was the ability to compute by decomposing a surface into elementary pieces. The analog of that here is a certain refinement of the MayerVietoris sequence. Consider cutting $\Sigma$ on a circle $S$ to decompose it into pieces $\Sigma_{L}$ and $\Sigma_{R}$ (Fig. 13a). We pick a cell decomposition of $\Sigma$ by polygons which restricts to cell decompositions of $S, \Sigma_{L}, \Sigma_{R}$, as in the figure. From this, one gets a short exact 
sequence of chain complexes,

$$
0 \rightarrow C_{S \bullet} \rightarrow C_{\Sigma_{L} \bullet} \oplus C_{\Sigma_{R} \bullet} \rightarrow C_{\Sigma \bullet} \rightarrow 0 .
$$

The long exact sequence of cohomology that follows from (4.29) implies that

$$
\operatorname{det} H_{*}(\Sigma, \operatorname{ad}(E))=\operatorname{det} H_{*}\left(\Sigma_{L}, \operatorname{ad}(E)\right) \otimes \operatorname{det} H_{*}\left(\Sigma_{R}, \operatorname{ad}(E)\right) \otimes\left(\operatorname{det} H_{*}(S, \operatorname{ad}(E))\right)^{-1} .
$$

Moreover, (4.29) is easily seen to be volume-exact, so the alternating product of the torsions is 1 [as in (4.14)]. If we denote the torsion of the chain complex that computes $H_{*}(W, \operatorname{ad}(E))$ as $\tau(W)$ (for $W=\Sigma, \Sigma_{L}, \Sigma_{R}$, or $S$ ), then

$$
\tau(\Sigma)=\tau\left(\Sigma_{L}\right) \otimes \tau\left(\Sigma_{R}\right) \otimes \tau(S)^{-1} .
$$

Equation (4.30), which asserts that the left- and right-hand sides of (4.31) lie in the same vector space, is necessary for (4.31) to make sense.

This can be repeated (Fig. 13b). If $\Sigma$ is an orientable surface of genus $g$, and we decompose $\Sigma$ into $2 g-2$ three holed spheres $W_{1}, \ldots, W_{2 g-2}$ by cutting on $3 g-3$ circles $S_{1}, \ldots, S_{3 g-3}$, then a repetition of the type of argument that led to (4.31) gives

$$
\tau(\Sigma)=\bigotimes_{i=1}^{2 g-2} \tau\left(W_{i}\right) \bigotimes_{j=1}^{3 g-3} \tau\left(S_{j}\right)^{-1}
$$

Thus to compute $\tau(\Sigma)$, we need only understand the torsions of flat connections on circles and three holed spheres.

We shall assume $G$ is connected, which ensures that any $G$ bundle $E$ is trivial over any of the $S_{i}$ or $\Sigma_{j}$. The type of computation that we will perform below sums over all possibilities for gluing along the $S_{i}$ and therefore means that the volume that we will compute will be the sum over all topological types of $G$ bundles of the volume of the moduli space of flat connections for that topological type.

\subsection{The Torsion of the Three Holed Sphere}

In this subsection, $\Sigma$ is a sphere with three holes. $G$ is a connected semi-simple gauge group. Semi-simplicity means that the center $Z(G)$ is a finite set; we set $G_{0}=G / Z(G)$. Semisimplicity also ensures that the generic flat connection on the three holed sphere is irreducible, with $H_{*}(\Sigma, \operatorname{ad}(E))$ vanishing except in dimension one. We let $\mathcal{N}$ denote the moduli space of irreducible flat connections on $\Sigma$. Then $H_{1}(\Sigma, \operatorname{ad}(E)) \cong T^{*}(\mathscr{N})$. The torsion hence defines a measure on $\mathscr{N}$ which we will call $\tau(\mathscr{N})$; we wish to study this.

It is useful to introduce the moduli space $\hat{N}$ of based irreducible flat connections on $\Sigma$. Thus, one picks a base point $P \in \Sigma$, and considers the moduli space $\hat{\mathscr{N}}$ of flat connections on $\Sigma$, up to gauge transformations that equal the identity at $P$. There is a natural projection $\varrho: \hat{N} \rightarrow \mathcal{N}$. The fiber is a copy of the adjoint group $G_{0}$. One has then a short exact sequence of cotangent spaces:

$$
0 \rightarrow T^{*} \mathscr{N} \rightarrow T^{*} \hat{\mathscr{N}} \rightarrow T^{*} G_{0} \rightarrow 0 .
$$

This sequence appears naturally in the following way. Pick a cell decomposition $X$ of $\Sigma$, of the type considered in the previous subsection, with $P$ as one of the zerocells. The choice of $X$ determines a chain complex $C_{\bullet}$ that computes $H_{*}(\Sigma, \operatorname{ad}(E))$. 


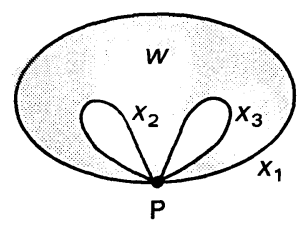

Fig. 14. A small cell decomposition of the three holed sphere

Let $C_{P}$ be the subcomplex of this consisting only of the chain group $V_{P}$ in dimension zero. It is a very short chain complex

$$
0 \rightarrow C_{P 0}=V_{P} \rightarrow 0
$$

with differential zero. $C_{p} \bullet$ has a natural embedding in $C_{\boldsymbol{\bullet}}$. Let $C_{\bullet}^{\prime}$ be the quotient, so one has a short exact sequence of complexes,

$$
0 \rightarrow C_{P \bullet} \rightarrow C_{\bullet} \rightarrow C_{\bullet}^{\prime} \rightarrow 0 .
$$

The long exact homology sequence derived from (4.35) reduces to

$$
0 \rightarrow H_{1}\left(C_{\bullet}\right) \rightarrow H_{1}\left(C_{\bullet}^{\prime}\right) \rightarrow H_{0}\left(C_{p_{\bullet}}\right) \rightarrow 0
$$

as all the other homology groups vanish. This sequence may be identified with (4.33), as $H_{1}\left(C_{\bullet}\right) \cong T^{*} \mathcal{N}, H_{1}\left(C_{\bullet}^{\prime}\right) \cong T^{*} \mathscr{N}^{\prime}$, and $H_{0}\left(C_{P \bullet}\right) \cong T^{*} G_{0}$.

With the usual measures on each chain group, the sequence (4.35) is volumeexact, so

$$
\tau\left(C_{\bullet}\right)=\tau\left(C_{\bullet}^{\prime}\right) \cdot \tau\left(C_{P_{\bullet}}\right) .
$$

Here we encounter a slight subtlety. As in the last section, $\tau\left(C_{\bullet}\right)$ and $\tau\left(C_{\bullet}^{\prime}\right)$ lie in $\operatorname{det} H_{1}\left(C_{\bullet}\right) \cong \operatorname{det} T^{*} \mathcal{N}$ and $\operatorname{det} H_{1}\left(C_{\bullet}^{\prime}\right) \cong \operatorname{det} T^{*} \hat{N}$, and so define measures on $\mathscr{N}$ and $\hat{\mathcal{N}}$. As for $\tau\left(C_{P 0}\right)$, however, it lies in $\left(\operatorname{det} H_{0}\left(C_{P}\right)\right)^{-1} \cong\left(\operatorname{det} T^{*} G_{0}\right)^{-1}$, and so transforms as the inverse of a measure on $G_{0}$. In fact, as the boundary operator of the very short complex $C_{P \bullet}$ is zero, $\tau\left(C_{P \bullet}\right)^{-1}$ is the natural Haar measure $\mu$ on $G_{0}$ determined by the metric on $\mathscr{G}$, as discussed in Sect. 4.1. Taking advantage of the fibering $\varrho: \hat{\mathcal{N}} \rightarrow \mathcal{N},(4.37)$ can be rewritten in the form

$$
\tau\left(C^{\prime}\right)=\varrho^{*}(\tau(C)) \cdot \mu .
$$

[In this paper, we regard measures as sections of appropriate real line bundles, and the product on the right-hand side of (4.38) is really a tensor product of such sections.] Equation (4.38) can also be inverted to give

$$
\tau(C)=\frac{1}{\operatorname{Vol}\left(G_{0}\right)} \cdot \varrho_{*}\left(\tau\left(C^{\prime}\right)\right),
$$

where the volume of $G_{0}$ is computed with the measure $\mu$.

We are thus reduced to computing $\tau\left(C^{\prime}\right)$, and this is elementary. The three holed sphere can be given a cell decomposition with one two cell $w$, three one cells $x_{1}, x_{2}$, and $x_{3}$, and one zero cell $P$ (Fig. 14). A irreducible flat connection can be described in a fashion that is unique up to a unique gauge transformation that is 1 at $P$ by giving its holonomies $U_{1}, U_{2}$, and $U_{3}$ around $x_{1}, x_{2}$, and $x_{3}$. They are arbitrary except for one relation

$$
U_{1} U_{2} U_{3}=1
$$


As this equation can be uniquely solved for $U_{3}$ in terms of $U_{1}$ and $U_{2}, \hat{N}$ is isomorphic to $G \times G$. We will see that $\tau\left(C^{\prime}\right)$ is the Haar measure on $G \times G$.

To describe the chain complex and the boundary operator in this situation (and similar situations that we will consider later) takes a little care, since $\operatorname{ad}(E)$ can be parallelized only over the interiors of the cells. Given a cell $u$, one defines $V_{u}$ to consist of parallel sections of $\operatorname{ad}(E)$ over the interior of $u$. If $t$ is such a section, $\partial t$ is computed by taking the limiting values of $t$ as one approaches the interiors of the various boundary cells. It might happen that some of the cells in the boundary of $u$ coincide (up to signs determined by the orientations); for instance, each end of the one-cells $x_{i}$ of Fig. 14 consists of the same zero-cell $P$. In this case, one simply adds the contributions at the various ends.

In the case at hand, the complex $C_{0}^{\prime}$ that computes the tangent space to $\hat{\mathcal{N}}$ is particularly simple to describe. $C_{\bullet}^{\prime}$ contains nonzero chain groups in dimensions two and one only (the zero dimensional chain group $V_{P}$ is precisely what is eliminated in passing from $C_{\bullet}$ to $C_{\bullet}^{\prime}$ ). In detail, $C_{2}^{\prime}=V_{w}$, and $C_{1}^{\prime}=\bigoplus_{i=1}^{3} V_{x_{i}}$. The boundary operator is as follows. For $s$ a parallel section of $\operatorname{ad}(E)$ over $w$,

$$
\partial s=\left.\left.\left.s\right|_{x_{1}} \oplus s\right|_{x_{2}} \oplus s\right|_{x_{3}} .
$$

To compute the torsion of this complex, let $E_{\bullet}$ be the subcomplex whose only chain group is $V_{x_{2}} \oplus V_{x_{3}}$ in dimension 1. Then $H_{1}(E)=T^{*}(G \times G)$. We have an exact sequence

$$
0 \rightarrow E_{\bullet} \rightarrow C_{\bullet}^{\prime} \rightarrow F_{\bullet} \rightarrow 0 \text {, }
$$

where $F_{0}$ is the complex

$$
0 \rightarrow V_{w} \rightarrow V_{x_{1}} \rightarrow 0 \rightarrow 0
$$

(in dimensions 2 and 1) with boundary operator $\partial s=\left.s\right|_{x_{1}}$. With the natural measures on $E_{\bullet}$ and $F_{\bullet},(4.42)$ is volume exact. $F_{\bullet}$ is acyclic, and its torsion is 1 . Hence $\tau\left(C_{\bullet}^{\prime}\right)=\tau\left(E_{\bullet}\right)$. However, $E_{\bullet}$ has zero boundary operator, so its torsion is the Haar measure on $G \times G$. This establishes our claim that $\tau\left(C_{0}^{\prime}\right)$ is equal to that Haar measure.

Having determined $\tau\left(C_{\bullet}^{\prime}\right)$, we can now in principle use (4.39) to determine $\tau\left(C_{\bullet}\right)$, by pushing down from $\hat{\mathscr{N}}$ to $\mathcal{N}$. It is not convenient to do this, however, because in contrast to $\hat{\mathscr{N}}, \mathcal{N}$ is awkward to describe explicitly. The following variant is more useful.

Let $L$ be the moduli space of conjugacy classes in $G$. Since we assume $G$ compact and connected, $L=T / W$ with $T$ the maximal torus and $W$ the Weyl group.

The association to a flat connection $A$ on the three holed sphere of the conjugacy classes $\Theta_{1}, \Theta_{2}$, and $\Theta_{3}$ of the monodromy around the three holes gives a natural map $\sigma: \mathcal{N} \rightarrow L \times L \times L$. We will determine $\sigma_{*}(\tau(C))$. According to (4.39), this is

$$
\sigma_{*}(\tau(C))=\frac{1}{\operatorname{Vol}\left(G_{0}\right)}(\varrho \sigma)_{*}\left(\tau\left(C^{\prime}\right)\right) .
$$

As $\alpha$ runs over all irreducible representations of $G$, the characters $\chi_{\alpha}(\Theta)$ are in an appropriate sense (a precise statement appears shortly) a basis of functions on $L$. Hence, to understand the measure $\sigma_{*}(\tau(C))$ on $L \times L \times L$, it suffices to compute

$$
W_{\alpha_{1}, \alpha_{2}, \alpha_{3}}=\int_{L \times L \times L} \sigma_{*}(\tau(C)) \prod_{i=1}^{3} \chi_{\alpha_{i}}\left(\Theta_{i}\right)
$$


for arbitrary $\alpha_{i}$. This is

$$
W_{\alpha_{1}, \alpha_{2}, \alpha_{3}}=\frac{1}{\operatorname{Vol} G_{0}} \int_{\mathscr{N}} \tau\left(C^{\prime}\right) \prod_{i=1}^{3}(\varrho \sigma)^{*} \chi_{\alpha_{i}}\left(\Theta_{i}\right) .
$$

Recalling that the monodromies obey $U_{1} U_{2} U_{3}=1$, and that $\tau\left(C^{\prime}\right)$ is Haar measure on $G \times G$, we get

$$
W_{\alpha_{1}, \alpha_{2}, \alpha_{3}}=\frac{1}{\operatorname{Vol} G_{0}} \int d U_{1} d U_{2} \chi_{\alpha 1}\left(U_{1}\right) \chi_{\alpha_{2}}\left(U_{2}\right) \chi_{\alpha_{3}}\left(U_{2}^{-1} U_{1}^{-1}\right),
$$

where $d U$ denotes Haar measure on $G$. This can be evaluated using the integral

$$
\int d U \chi_{\alpha}(U A) \chi_{\beta}\left(U^{-1} B\right)=\delta_{\alpha, \beta} \frac{\chi_{\alpha}(A B)}{\operatorname{dim} \alpha} \cdot \operatorname{Vol}(G)
$$

which for similar reasons played a key role in Sects. 2.3-4. [In Sect. 2.3, we defined the Haar measure so that $\operatorname{Vol}(G)=1$.] Using (4.48) twice and recalling that $\chi_{\alpha}(1)=\operatorname{dim} \alpha, \operatorname{Vol}(G) / \operatorname{Vol}\left(G_{0}\right)=\# Z(G)$, we get

$$
W_{\alpha_{1}, \alpha_{2}, \alpha_{3}}=\delta_{\alpha_{1}, \alpha_{2}, \alpha_{3}} \cdot \frac{1}{\operatorname{dim} \alpha} \cdot \operatorname{Vol}(G) \cdot \# Z(G) .
$$

Actually, it is now easy to determine the measure $\sigma_{*}(\tau(C))$ that gives rise to (4.49). Let $\mu$ be Haar measure on $G, u: G \rightarrow L$ the natural projection (from a group element to its conjugacy class), and $v=u_{*}(\mu)$. The basic result on orthonormality of characters asserts that

$$
\int_{L} d v \overline{\chi_{\alpha}(\Theta)} \chi_{\beta}(\Theta)=\delta_{\alpha \beta} \cdot \operatorname{Vol}(G)
$$

The measure $\sigma_{*}(\tau(C))$ that leads to the evaluation (4.49) of (4.45) is hence

$$
\sigma_{*}(\tau(C))=\frac{\# Z(G)}{\operatorname{Vol}(G)^{2}} \cdot \sum_{\alpha} \frac{1}{\operatorname{dim} \alpha} \prod_{i=1}^{3} \chi_{\alpha}\left(\Theta_{i}\right) \cdot v_{1} v_{2} v_{3},
$$

where $v_{i}, i=1 \ldots 3$ is the measure $v$ on the $i^{\text {th }}$ copy of $L$ in $L \times L \times L$, and $v_{1} v_{2} v_{3}$ is hence the product measure on $L \times L \times L$.

\subsection{The Torsion of a Circle}

We now let $\mathscr{2}$ denote the moduli space of flat connections on a circle $S$. A connection on $S$ (any connection is flat) is determined up to gauge transformation by the conjugacy class of the holonomy, which is arbitrary. Hence $\mathscr{Q}$ is isomorphic to the moduli space $L=T / W$ of conjugacy classes.

Decompose the Lie algebra $\mathscr{G}$ of $G$ as $\mathscr{G}=\mathscr{T} \oplus \mathscr{R}$, where $\mathscr{T}$ is the Lie algebra of $T$, and $\mathscr{R}$ is the orthocomplement. $T$ acts on $\mathscr{R}$ by restricting the adjoint action of $G$ on $\mathscr{G}$. An important function on $T$ (the square of the denominator in the Weyl character formula) is

for $U \in T$.

$$
F(U)=\operatorname{det}_{\mathscr{R}}(1-U)
$$

On $L$, as we noted in Sect. 4.1, there are two natural measures. The metric on $\mathscr{G}$ restricts to a metric on $\mathscr{T}$, which determines a Haar measure $v_{0}$ on $T$; and we use 


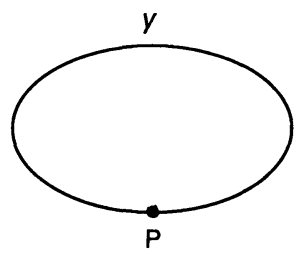

Fig. 15. A simple cell decomposition of the circle

the same name for the measure on $L$ determined by the local isomorphism of $L$ and $T$. On the other hand, Haar measure on $G$ pushes down (under the natural map from a group element to its conjugacy class) to a measure $v$ on $L$. A formula due to Weyl asserts

$$
v=\frac{\operatorname{Vol}(G)}{\operatorname{Vol}(T)} \cdot v_{0} \cdot F(U)
$$

(see [19, Chap. IV, Formula (1.11)]).

A generic conjugacy class - and hence a generic connection on $S$ - has a stabilizer $F$ isomorphic to $T \cdot H^{0}(S, \operatorname{ad}(E))$ is the Lie algebra of this stabilizer and so is isomorphic to $\mathscr{T} \cdot H^{1}(S, \operatorname{ad}(E))$ is the tangent space to $\mathscr{Q} \cong T / W$ and can again be identified with $\mathscr{T}$. The torsion $\tau$ of a connection $A$ on $S$ takes values (according to the general discussion of torsion) in the real line

$$
\operatorname{det} H_{1}(S, \operatorname{ad}(E)) \otimes\left(\operatorname{det} H_{0}(S, \operatorname{ad}(E))\right)^{-1} .
$$

From what we have just said, this ratio is canonically trivial. However, it will be more natural to write $\tau$ the way it naturally appears in (4.54), as a ratio of two measures on 2. Indeed, we will see that

$$
\tau(S)=\frac{v}{v_{0}} \cdot \frac{\operatorname{Vol}(T)}{\operatorname{Vol}(G)} .
$$

In fact, $\operatorname{ad}(E)$ has a decomposition $\operatorname{ad}(E)=\operatorname{ad}(E)_{0} \oplus \operatorname{ad}(E)_{1}$, where $\operatorname{ad}(E)_{0}$ is the sub-bundle of $\operatorname{ad}(E)$ invariant under the stabilizer $F$ of the flat connection, and $\operatorname{ad}(E)_{1}$ is the orthocomplement. (Note that $\operatorname{ad}(E)_{0}$ is the trivial flat bundle $\operatorname{ad}(E)_{0}$ $=S \times \mathscr{T}$.) If we compute the torsion of a connection on $S$ by picking a cell decomposition and studying the corresponding chain complex $C_{0}$, then one has $C_{\bullet}=C_{0} \oplus C_{1}$, where $C_{0}$ (which is the $T$ invariant part) and $C_{1} \bullet$ (which is the orthocomplement) compute $H_{*}\left(S, \operatorname{ad}(E)_{0}\right)$ and $H_{*}\left(S, \operatorname{ad}(E)_{1}\right)$, respectively. Hence

$$
\tau(S)=\tau_{0} \cdot \tau_{1},
$$

where $\tau_{0}$ and $\tau_{1}$ are the torsions of $\operatorname{ad}(E)_{0}$ and $\operatorname{ad}(E)_{1}$, respectively.

In fact, we can take a very simple cell decomposition of $S$ (Fig. 15) with one 1-cell $y$ and one 0 -cell $P$. The corresponding chain complex $D_{\bullet}$ that computes $H_{*}(S, \mathbb{R})$ has a one dimensional chain group in dimension zero, a one dimensional chain group in dimension one, and boundary operator zero (as $\partial y=0$ ). Since $\operatorname{ad}(E)_{0} \cong S \times \mathscr{T}$, the chain complex $C_{0}$. that computes $H_{*}\left(S, \operatorname{ad}(E)_{0}\right)$ is $C_{0} \bullet=D \otimes \mathscr{T}$. In particular, the boundary operator of $C_{0}$ is zero, the zero and one dimensional chain groups $V_{P}$ and $V_{\gamma}$ are copies of $\mathscr{T}$ with its natural measure, and so

$$
\tau_{0}=\tau\left(C_{00}\right)=\frac{v_{0}}{v_{0}} .
$$


As for $\operatorname{ad}(E)_{1}$, it is acyclic, so the torsion will be a number or more exactly a function on 2 . The chain complex $C_{1}$ that computes $H_{*}\left(S, \operatorname{ad}(E)_{1}\right)$ has one and zero dimensional chain groups $V_{y}$ and $V_{P}$ consisting of parallel sections of $\operatorname{ad}(E)_{1}$ over the interior of $y$ and over $P$, respectively. $V_{y}$ and $V_{P}$ can each be identified with $\mathscr{R}$ with its natural measure. With appropriate parallelizations of $E$ over $y$ and over the interior of $w$, the boundary map $\partial: V_{y} \rightarrow V_{P}$, if $E$ is defined by a flat connection with holonomy $U$, is

$$
\partial s=s-U s U^{-1} .
$$

[To see this, first pick a basis $s_{i}$ for $E_{\mathrm{P}}$. There is a unique basis of parallel sections $s_{i}^{\prime}$ of $E$ over the interior of $y$ which, as one approaches $\partial y$ on the left, coincide with the $s_{i}$. As one approaches $\partial y$ on the right, $s_{i}^{\prime}$ will approach $U s_{i} U^{-1}, U$ being the holonomy. Allowing for the opposite orientations at the two ends to determine the signs, one gets (4.58).] The torsion is hence

$$
\tau_{1}=\operatorname{det}_{\mathscr{R}}(1-U)=F(U) .
$$

Combining (4.59), (4.56), and (4.53), we arrive at the claimed formula (4.55) for the torsion of a connection on the circle.

\subsection{The Volume of $\mathscr{M}$ for $\Sigma$ Orientable}

Now we can finally put the pieces together and compute the volume of the moduli space $\mathscr{M}$ of flat connections on an orientable surface $\Sigma$.

We decompose $\Sigma$ as a union of $2 g-2$ three holed spheres $W_{i}$, joined along $3 g-3$ circles $S_{\gamma}$. Let $\mathscr{N}_{i}$ and $\mathscr{Q}_{\gamma}$ be the moduli space of flat connections on $W_{i}$ and $S_{\gamma}$. Given a flat connection $A$ on a $G$ bundle $E$ over $\Sigma$, we write $\tau(\Sigma), \tau\left(W_{i}\right)$, or $\tau\left(S_{\gamma}\right)$ for the torsion of $\operatorname{ad}(E)$ restricted to the space in question. Each $\tau$ is a vector in an appropriate real line. The generalized Mayer-Vietoris formula (4.32) asserts that

$$
\tau(\Sigma)=\bigotimes_{i} \tau\left(W_{i}\right) \bigotimes_{\gamma} \frac{1}{\tau\left(S_{\gamma}\right)} .
$$

There is a natural map

$$
\mathscr{M} \rightarrow \prod_{i} \mathscr{N}_{i}
$$

that maps a flat connection $A$ on $\Sigma$ to $\left(\left.A\right|_{W_{1}}, \ldots,\left.A\right|_{W_{2 g-2}}\right)$. This map is not surjective, since if $W_{i} \cap W_{j}$ contains a circle $S_{\gamma}$, then $\left.A\right|_{W_{i}}$ and $\left.A\right|_{W_{j}}$ are automatically isomorphic when restricted to $S_{\gamma}$ - while $\prod_{i} \mathcal{N}_{i}$ would consist of flat connections on the $W_{i}$ chosen independently with no such restriction. Let $\mathcal{N}^{\prime}$ be the subspace of $\prod_{i} \mathscr{N}_{i}$ consisting of gauge equivalence classes of families of flat connections $A_{(i)}$ on the $W_{i}$ such that $A_{(i)}$ and $A_{(j)}$ are gauge equivalent on $W_{i} \cap W_{j}$. Obviously, the image of $\mathscr{M}$ lies in $\mathscr{N}^{\prime}$.

The natural map $\mathscr{M} \rightarrow \mathscr{N}^{\prime}$ is surjective, a fact that can be seen as follows. Given a family of flat connections $A_{(i)}$ on $W_{i}$, defining a point in $\mathscr{N}^{\prime}$, proceed as follows. Each circle $S_{\gamma}$ appears as a component of the boundary of two $W$ 's (or appears twice in the boundary of one $W)$. Let $i(\gamma)$ and $i^{\prime}(\gamma)$ be the two values of $i$ for which $S_{\gamma} \subset \partial W_{i}$. By definition of $\mathcal{N}^{\prime}, A_{(i(\gamma))}$ and $A_{\left(i^{\prime}(\gamma)\right)}$ are gauge equivalent when restricted to $S_{\gamma}$. So let $\phi_{\gamma}$ be a gauge equivalence between them:

$$
\phi_{\gamma}:\left.\left.A_{(i(\gamma))}\right|_{S_{\gamma}} \cong A_{\left(i^{\prime}(\gamma)\right)}\right|_{S_{\gamma}} .
$$


Using the $\phi_{\gamma}$ to glue together the bundles and connections on the various $W_{i}$, we get a flat connection $A$ on $\Sigma$ that maps to $\left(A_{(1)}, \ldots, A_{(2 g-2)}\right)$ under the natural map $\mathscr{M} \rightarrow \mathscr{N}^{\prime}$; this shows that that map is surjective.

However, the map $\mathscr{M} \rightarrow \mathcal{N}^{\prime}$ is not one to one. The reason is that the $\phi_{\gamma}$ are not unique. Let $A$ be a flat connection on $\Sigma$. For each $\gamma$, let $F_{y}$ be the group of gauge transformations that leaves $\left.A\right|_{S_{\gamma}}$ invariant. $F_{\gamma}$ is determined up to isomorphism by the conjugacy class of the holonomy around $S_{\gamma}$, and for generic $A$, each $F_{\gamma}$ is a copy of the maximal torus $T$. Throwing away a set of measure zero, we henceforth consider only $A$ 's for which this is true for each $\gamma$, and we write $T_{\gamma}$ for $F_{\gamma}$. The group $T^{\prime}=\prod_{\gamma} T_{\gamma}$ acts on the fiber of $\mathscr{M} \rightarrow \mathscr{N}^{\prime}$ containing $A$ as follows. Letting $A_{(i)}=\left.A\right|_{W_{i}}$, $A$ can be considered to arise by gluing together the $A_{(i)}$ with some gluing data, as in (4.62). Given an element $\prod t_{\gamma} \in T^{\prime}=\prod T_{\gamma}$, and transforming the gluing data by

$$
\phi_{\gamma} \rightarrow \phi_{\gamma} t_{\gamma} \text {, }
$$

we get a new set of gluing data that gives a family of flat connections parametrized by the $t_{\gamma}$.

The group $T^{\prime}$ acts transitively on the fibers of $\mathscr{M} \rightarrow \mathcal{N}^{\prime}$, since if $\phi_{\gamma}:\left.A\right|_{(i(\gamma))}$ $\left.\cong A\right|_{\left(i^{\prime}(\gamma)\right)}$ and $\tilde{\phi}_{\gamma}:\left.\left.A\right|_{(i(\gamma))} \cong A\right|_{\left(i^{\prime}(\gamma)\right)}$ are two families of identifications of $\left.A\right|_{(i(\gamma))}$ and $\left.A\right|_{\left(i^{\prime}(\gamma)\right)}$, then $\tilde{\phi}_{\gamma}^{-1} \phi_{\gamma}$ are isomorphisms of $\left.A\right|_{(i(\gamma))}$ and thus elements of $T_{\gamma}$. However, in general $T^{\prime}$ does not act freely on the fibers. It may happen that for some $t_{\gamma}$, the change of gluing data (4.63) does not affect the gauge equivalence class of the glued connection. This will occur precisely if there exist automorphisms $u_{i}$ of $\left.A\right|_{W_{i}}$ such that the transformation (4.63) of the gluing data can be absorbed in the automorphisms $u_{i}$ of the objects that are being glued. A family of automorphisms $u_{i}$ of $\left.A\right|_{W_{i}}$ will transform the $t_{\gamma}$ in (4.63) by

$$
t_{\gamma} \rightarrow u_{i^{\prime}(\gamma)} t_{\gamma} u_{i(\gamma)}^{-1} \text {. }
$$

If $\left.A\right|_{W_{i}}$ is irreducible, then its only automorphisms are constant gauge transformations by elements of the center of $G$. Throwing away a set of measure zero, we can henceforth assume that each $\left.A\right|_{W_{i}}$ is irreducible. This being so, the automorphisms $\left(u_{1}, \ldots, u_{2 g-2}\right)$ in (4.64) are all elements of the center $Z(G)$. There are \#Z(G) ${ }^{2 g-2}$ ways to choose this number of elements of $Z(G)$, but since the ratios $u_{i^{\prime}(\gamma)} u_{i(y)}^{-1}$ that appear in (4.64) are all one if the $u^{\prime}$ 's are all equal, those ratios have $\# Z(G)^{2 g-3}$ possible values. The set of those values is a subgroup $\mathscr{Z}$ of $T^{\prime}$ of order $\# Z(G)^{2 g-3}$, and in the $T^{\prime}$ action on the fibers of $\mathscr{M} \rightarrow \mathscr{N}^{\prime}$, the stabilizer of a generic point is precisely $\mathscr{Z}$.

Let $T^{\prime \prime}=T^{\prime} / \mathscr{Z} . T^{\prime \prime}$ acts freely on the fibers of $\mathscr{M} \rightarrow \mathscr{N}^{\prime}$, so we have a fibration

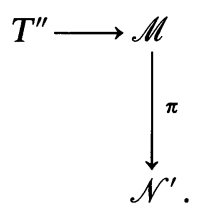

Any measure on $\mathscr{M}$, such as the torsion measure $\tau$, can be factored as

$$
\tau=\lambda \cdot \pi^{*}\left(\tau^{\prime}\right),
$$

where $\lambda$ is Haar measure on $T^{\prime \prime}$ and $\tau^{\prime}$ is some measure on $\mathcal{N}^{\prime}$. Integrating over the fibers of $\pi$, and remembering from (4.15) that what we have called the volume of $\mathscr{M}$ 
is defined with a factor of $(2 \pi)^{-\operatorname{dim} \mathscr{M}}$, we get

$$
\operatorname{Vol} \mathscr{M}=\frac{\operatorname{Vol}(T)^{3 g-3}}{(2 \pi)^{\operatorname{dim} \mathscr{M}} \cdot \# Z(G)^{2 g-3}} \cdot \int_{\mathcal{S}^{\prime}} \tau^{\prime}
$$

Final Evaluation. There is a natural map $v: \mathscr{N}^{\prime} \rightarrow \prod_{\gamma} \mathscr{Q}_{\gamma}$ that maps a family of connections $A_{(i)}$ on $W_{i}$, with compatible restrictions $\left.A\right|_{S_{\gamma}}$, to their restrictions $\left(\left.A\right|_{S_{1}}, \ldots,\left.A\right|_{S_{3 g-3}}\right)$. We can rewrite $(4.67)$ as

$$
\operatorname{Vol}(\mathscr{M})=\frac{\operatorname{Vol}(T)^{3 g-3}}{(2 \pi)^{\operatorname{dim} \mathscr{M}} \# Z(G)^{2 g-3}} \cdot \int_{2_{1} \times \ldots \times 2_{3 g-3}} v_{*} \tau^{\prime} .
$$

It remains to determine $v_{*} \tau^{\prime}$ and compute the integral. This can be done as follows, starting with the generalized Mayer-Vietoris formula,

$$
\tau(\Sigma)=\bigotimes_{i} \tau\left(W_{i}\right) \bigotimes_{\gamma} \frac{1}{\tau\left(S_{\gamma}\right)} .
$$

We use (4.51) for $\tau\left(W_{i}\right)$ (or rather its push-forward by $v$ ) and (4.55) for $\tau\left(S_{\gamma}\right)$. In the denominator of (4.55) appears the Haar measure $v_{0}$, which is naturally interpreted as Haar measure on the group $T_{\gamma}$ of automorphisms of $\left.A\right|_{S_{\gamma}}$. The $v_{0, \gamma}$ actually appear in the numerator in (4.69) [since they are in the denominator in (4.55)], and their product $v_{0,1} \ldots v_{0,3 g-3}$ is precisely the Haar measure $\lambda$ on $T^{\prime \prime}$ that appears in (4.66). Hence, to compute $\tau^{\prime}$, we just evaluate the Mayer-Vietoris product (4.69) suppressing the $v_{0}$ 's in (4.55).

Actually, what we will compute is the pushforward $v_{*}\left(\tau^{\prime}\right)$, a measure on $\prod_{\gamma} \mathscr{Q}_{\gamma}$. Apart from the $v_{0}$ 's that we are now suppressing, the only explicit measures that appear in (4.51) and (4.55) are factors of $v$. We recall that $v$ is the measure on $L=T / W$ that comes by pushing down Haar measure on $G$. For $\gamma=1, \ldots, 3 g-3$, we get two factors of the Haar measure $v_{\gamma}$ in the numerator of the Mayer-Vietoris formula (since each $S_{\gamma}$ is in the boundary of two $W_{i}$ ), and one in the denominator (from the formula for $\tau\left(S_{\gamma}\right)$ ). Altogether, then, $v_{*}\left(\tau^{\prime}\right)$ is $\prod_{\gamma} v_{\gamma}$ times some factors appearing in (4.51) and (4.55). Explicitly,

$$
\begin{aligned}
v_{*}\left(\tau^{\prime}\right)= & \left.(\operatorname{Vol}(G))^{-2} \cdot \# Z(G)\right)^{2 g-2} \cdot\left(\frac{\operatorname{Vol}(G)}{\operatorname{Vol}(T)}\right)^{3 g-3} \\
& \times \prod_{\gamma} v_{\gamma} \cdot \sum_{\alpha_{1}, \ldots, \alpha_{2 g-2}} \cdot \prod_{s_{\gamma} \subset \partial W_{i}} \chi_{\alpha_{i}}^{(-)}\left(\Theta_{\gamma}\right) .
\end{aligned}
$$

Here we pick an orientation around each $S_{\gamma}, \Theta_{\gamma}$ is the holonomy of $A$ around $S_{\gamma}$ with this orientation, and $\chi_{\alpha_{i}}^{(-)}\left(\Theta_{\gamma}\right)$ is equal to $\chi_{\alpha_{i}}\left(\Theta_{\gamma}\right)$ or $\overline{\chi_{\alpha_{i}}\left(\Theta_{\gamma}\right)}$ depending on whether the chosen orientation of $S_{\gamma}$ agrees with its induced orientation as a component of $\partial S_{\gamma}$.

Finally, we evaluate (4.68), using

$$
\int_{L} d v \cdot \chi_{\alpha}(\Theta) \overline{\chi_{\beta}(\Theta)}=\delta_{\alpha, \beta} \cdot \operatorname{Vol}(G)
$$

to perform the integral over the $\mathscr{Q}_{\gamma}$. We get simply

$$
\operatorname{Vol}(\mathscr{M})=\frac{\# Z(G) \cdot(\operatorname{Vol}(G))^{2 g-2}}{(2 \pi)^{\operatorname{dim} \mathscr{M}}} \cdot \sum_{\alpha} \frac{1}{(\operatorname{dim} \alpha)^{2 g-2}},
$$

where $\alpha$ runs over all isomorphism classes of irreducible representations of $G$. 
For example, for $G=S U(2), \# Z(G)=2$, $\operatorname{Vol}(S U(2))=2^{5 / 2} \pi^{2}$ with our definitions (as was explained in Sect. 4.1), $\operatorname{dim} \mathscr{M}=6 \mathrm{~g}-6$, and the dimensions of the irreducible representations are $1,2,3, \ldots$, so

$$
\operatorname{Vol}(\mathscr{M}(S U(2)))=2 \cdot \frac{1}{\left(2 \pi^{2}\right)^{g-1}} \cdot \sum_{n=1,2,3 \ldots} \frac{1}{n^{2 g-2}} .
$$

For $G=S O(3), \# Z(G)=1, \operatorname{Vol}(S O(3))=2^{3 / 2} \pi^{2}, \operatorname{dim} \mathscr{M}=6 g-6$, and the dimensions of irreducible representations are $1,3,5, \ldots$, so

$$
\operatorname{Vol}(\mathscr{M}(S O(3)))=\frac{1}{\left(8 \pi^{2}\right)^{g-1}} \cdot \sum_{n=1,3,5, \ldots} \frac{1}{n^{2 g-2}} .
$$

These results agree, of course, with the earlier formulas (3.11) and (3.29) based on the Verlinde formula (and they agree within the by now standard renormalization factor with the quantum Yang-Mills theory calculation of Sects. 2.3-4).

\subsection{Unorientable Surfaces}

We will now very briefly extend these considerations to compute the volumes of moduli spaces of flat connections on unorientable Riemann surfaces.

First, we consider a surface $\Sigma_{g, s}$ which is the connected sum of a surface of genus $g$ with $s$ Klein bottles. Such a surface can be constructed by gluing together $2 g-2+2 s$ three holed spheres along circles $S_{\gamma}$, with orientation-reversing diffeomorphisms of $s$ of the circles prior to the gluing (recall Fig. 6). The computation can hence be carried out using precisely the building blocks that we have just considered. Only one novelty arises. An orientation-reversing diffeomorphism replaces a character $\chi_{\alpha}\left(\Theta_{\gamma}\right)$ by the conjugate character $\chi_{\bar{\alpha}}\left(\Theta_{\gamma}\right)=\overline{\chi_{\alpha}\left(\Theta_{\gamma}\right)}$ $(\bar{\alpha}$ is the complex conjugate representation of $\alpha$ ). The absence of a consistent orientation of the surface means that at one or more points in the computation, one will encounter not

$$
\int_{L} d v \overline{\chi_{\alpha}(\Theta)} \chi_{\alpha}(\Theta)=\operatorname{Vol}(G)
$$

but

$$
\int_{L} \overline{\chi_{\alpha}(\Theta)} \chi_{\bar{\alpha}}(\Theta)=\delta_{\alpha, \bar{\alpha}} \operatorname{Vol}(G) .
$$

( $\delta_{\alpha, \bar{\alpha}}=1$ if $\alpha$ and $\bar{\alpha}$ are isomorphic and zero otherwise). So repeating the earlier steps, the volume of the moduli space of the connected sum of a surface of genus $g$ with $s$ Klein bottles is

$$
\operatorname{Vol}(\mathscr{M})=\frac{\# Z(G) \cdot(\operatorname{Vol}(G))^{2 g-2+2 s}}{(2 \pi)^{\operatorname{dim} \mathscr{M}}} \cdot \sum_{\alpha=\bar{\alpha}} \frac{1}{(\operatorname{dim} \alpha)^{2 g-2+2 s}} .
$$

The Projective Plane. Now we consider the more difficult case of a connected sum with copies of $R P^{2}$. This case cannot be reduced to gluing together three holed spheres, so we actually must make a new computation of torsions. We let $\Sigma^{\prime}$ be $R P^{2}$ with an open ball removed; $\partial \Sigma^{\prime}$ is a circle $S$. $\mathscr{P}$ and $\mathscr{Q}$ will denote the moduli spaces of flat connections on $\Sigma^{\prime}$ and on $S$.There is a natural map $u: \mathscr{P} \rightarrow \mathscr{Q}$ by restriction of a flat connection to the boundary. 


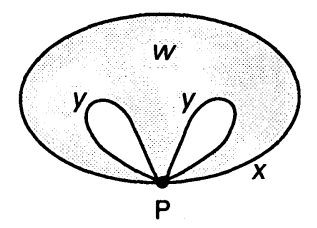

Fig. 16. A simple cell decomposition of $R P^{2}$ with a ball removed

In Fig. 16, we indicate a simple cell decomposition of $\Sigma^{\prime}$, with a two cell $w$, one cells $x$ and $y$ ( $y$ appears twice in the picture; the two copies are identified to make $\Sigma^{\prime}$ ), and a zero cell which is the base point $P$. The holonomies $U$ and $V$ of a flat connection around $y$ and $x$ obey one relation

$$
V=U^{2} \text {. }
$$

Since $V$ is thus determined by $U$, a flat connection on $\Sigma^{\prime}$ is determined up to gauge transformation by the conjugacy class of $U$. Thus, the moduli space $\mathscr{P}$ of flat connections on $\Sigma^{\prime}$, like the moduli space $\mathscr{Q}$ of flat connections on $S$, is a copy of $L=T / W$. However, the map $u: \mathscr{P} \rightarrow \mathscr{Q}$ is not the identity; it is the map $U \rightarrow V=U^{2}$. The stabilizer of a generic flat connection on $\Sigma^{\prime}$ is a copy of $T$, the maximal torus, that commutes with $U$ (and $V$ ).

The torsion of a flat connection on $\Sigma^{\prime}$ is a vector

$$
\tau \in \operatorname{det} H_{1}\left(\Sigma^{\prime}, \operatorname{ad}(E)\right) \otimes \frac{1}{\operatorname{det} H_{0}\left(\Sigma^{\prime}, \operatorname{ad}(E)\right)} .
$$

As for the denominator, $H_{0}\left(\Sigma^{\prime}, \operatorname{ad}(E)\right)$ is generically isomorphic to $H_{0}(S, \operatorname{ad}(E))$, with both of them being the Lie algebra $\mathscr{T}$ of the stabilizer $T$. Since $H_{0} \neq 0$, the torsion is not a measure on $\mathscr{P}$, but rather we can write

$$
\tau=\frac{\tilde{\tau}}{v_{0}}
$$

with $\tilde{\tau}$ a measure on $\mathscr{P}$, and $v_{0}$ the Haar measure on $T$.

As in discussing the three holed sphere, it is convenient to introduce the moduli space $\widehat{\mathscr{P}}$ of based flat connections on $\Sigma^{\prime}$. There is a generic fibering $r: \widehat{\mathscr{P}} \rightarrow \mathscr{P}$ with fiber $G / T$. The fiber is $G / T$ because $T$ is the stabilizer of a generic flat connection on $\Sigma^{\prime}$. Considering the natural projection $s: G \rightarrow G / T$, there is a measure $\mu^{\prime}$ on $G / T$ such that

$$
\mu=s^{*}\left(\mu^{\prime}\right) \cdot v_{0},
$$

where $\mu$ is Haar measure on $G$ and $v_{0}$ is Haar measure on $T$. Then

$$
\int_{G / T} d \mu^{\prime}=\frac{\operatorname{Vol}(G)}{\operatorname{Vol}(T)} \text {. }
$$

As in the discussion of the three holed sphere, let $C_{\bullet}$ be the complex, determined by a cell decomposition of $\Sigma^{\prime}$, that computes $H_{*}\left(\Sigma^{\prime}, \operatorname{ad}(E)\right)$, let $C_{P \bullet}$ be the subcomplex supported at the base point $P \in \Sigma^{\prime}$, and let $C_{\bullet}^{\prime}$ be the quotient complex:

$$
0 \rightarrow C_{P \bullet} \rightarrow C_{\bullet} \rightarrow C_{\bullet}^{\prime} \rightarrow 0 \text {. }
$$

$C_{\bullet}^{\prime}$ is acyclic except in dimension one, and $H_{1}\left(C_{\bullet}^{\prime}\right) \cong T^{*} \hat{P}$, so the torsion $\hat{\tau}=\tau\left(C_{\bullet}^{\prime}\right)$ is a measure on $\widehat{\mathscr{P}} . C_{P \bullet}$ is acyclic except in dimension zero, so its torsion takes values in 
$\operatorname{det}^{-1} H_{0}\left(C_{P \bullet}\right) \cong \operatorname{det} \mathscr{G}$. Indeed, identifying $\mathscr{G}$ with the tangent space to $G$, the torsion of $C_{\bullet}$ is just $\mu^{-1}, \mu$ being Haar measure on $G$. The volume-exactness of the exact sequence (4.83) implies that $\tau=\hat{\tau} \cdot \mu^{-1}$. If as in (4.80) we write $\tau=\tilde{\tau} / v_{0}$, and as in (4.81) we write $\mu^{\prime}=\mu / v_{0}$, then we have

Hence

$$
\tilde{\tau}=\frac{\hat{\tau}}{\mu^{\prime}}
$$

$$
u_{*}(\tilde{\tau})=\frac{1}{\operatorname{Vol}(G / T)}(u r)_{*}(\hat{\tau})=\frac{\operatorname{Vol}(T)}{\operatorname{Vol}(G)}(u r)_{*}(\hat{\tau}) .
$$

So to compute $u_{*}(\tilde{\tau})$, we just need to determine $\hat{\tau}$.

If we use the cell decomposition of Fig. 16, the reduced complex $C^{\prime}$ whose torsion is $\hat{\tau}$ has chain groups $C_{2}^{\prime}=V_{w}, C_{1}^{\prime}=V_{x} \oplus V_{y}$, and $C_{0}^{\prime}=0$. Let $F_{\bullet}$ be the subcomplex of $C^{\prime}$ whose boundary operator is zero and whose only nonzero chain group is $F_{1}=V_{y}$. Let $G_{\bullet}$ be the quotient:

$$
0 \rightarrow F_{\bullet} \rightarrow C_{\bullet}^{\prime} \rightarrow G_{\bullet} \rightarrow 0 \text {. }
$$

It is easy to see that $G_{\bullet}$ is an acyclic complex with torsion one. From the volumeexact sequence (4.86), it follows that $\hat{\tau}=\tau\left(C_{\bullet}^{\prime}\right)=\tau\left(F_{0}\right)$. As $F_{\bullet}$ has zero boundary operator, $\tau\left(F_{\bullet}\right)$ is just the natural measure on $F_{1}$. But $F_{1} \cong \mathscr{G} \cong T \widehat{\mathscr{P}}$, so $\hat{\tau}$ is just Haar measure on $\mathscr{P} \cong G$.

Together with (4.85), this gives a description of $u_{*}(\tilde{\tau})$, but not yet in a convenient form. For any representation $\alpha$ of $G$, let

$$
c_{\alpha}=\int_{2} u_{*}(\tilde{\tau}) \chi_{\alpha}(V) \text {, }
$$

where we recall that $V$ is the holonomy around the boundary. From (4.85), this is

$$
c_{\alpha}=\frac{\operatorname{Vol}(T)}{\operatorname{Vol}(G)} \int_{\hat{\mathscr{P}}} \hat{\tau} \cdot \chi_{\alpha}(V) .
$$

We have determined in the foregoing that $\widehat{\mathscr{P}} \cong G$, that $V=U^{2}$, and that $\hat{\tau}$ is just Haar measure on $U$. So

$$
c_{\alpha}=\frac{\operatorname{Vol}(T)}{\operatorname{Vol}(G)} \int d U \chi_{\alpha}\left(U^{2}\right)
$$

The integral on the right also arose in Sect. 2.4, and is

$$
\int d U \chi_{\alpha}\left(U^{2}\right)=f_{\alpha} \cdot \operatorname{Vol}(G)
$$

where $f_{\alpha}$ is $1,-1$, or 0 depending on whether the representation $\alpha$ admits a symmetric invariant bilinear form, an antisymmetric invariant bilinear form, or no invariant bilinear form at all. So

$$
c_{\alpha}=\operatorname{Vol}(T) \cdot f_{\alpha} .
$$

This evaluation of the integrals (4.87), together with the usual orthonormality of the characters, gives a more convenient description of the measure $u_{*}(\tilde{\tau})$ :

$$
u_{*}(\tilde{\tau})=\frac{\operatorname{Vol}(T)}{\operatorname{Vol}(G)} \cdot v \cdot \sum_{\alpha} f_{\alpha} \chi_{\alpha}(\Theta),
$$

with $\Theta$ the conjugacy class of the holonomy $V$ around the boundary of $\Sigma^{\prime}$. 
Now, we can compute the volume of the moduli space $\mathscr{M}_{g, s, r}$ of flat connections on the connected sum $\Sigma_{g, s, r}$ of an orientable surface of genus $g$ with $s$ Klein bottles and $r$ copies of the projective plane. Repeating all of the previous steps, one finds (for $r>0$ )

$$
\operatorname{Vol}\left(\mathscr{M}_{g, s, r}\right)=\# Z(G) \cdot \frac{\operatorname{Vol}(G)^{2 g-2+2 s+r}}{(2 \pi)^{\operatorname{dim} \mathscr{M}_{g, s, r}}} \sum_{\alpha} \frac{f_{\alpha}^{r}}{(\operatorname{dim} \alpha)^{2 g-2+2 s+r}} .
$$

[For $r=0$ and $s>0$, the sum over $\alpha$ must be restricted to representations for which $\alpha=\bar{\alpha}$. This restriction is built into (4.93) for $r>0$ as $f_{\alpha}=0$ unless $\alpha=\bar{\alpha}$.]

For example, let $G=S U(2)$. For $n=1,2,3, \ldots$, the $n$ dimensional representation has $f_{n}=(-1)^{n+1}$. The dimension of $\mathscr{M}_{g, s, r}$ is $6 g-g+6 s+3 r$. For $r$ odd, we get

$$
\operatorname{Vol}\left(\mathscr{M}_{g, s, r}(S U(2))\right)=\frac{2}{\left(2 \pi^{2}\right)^{g-1+s+r / 2}} \cdot \sum_{n=1}^{\infty} \frac{(-1)^{n+1}}{n^{2 g-2+2 s+r}} .
$$

One has

$$
\sum_{n=1}^{\infty} \frac{(-1)^{n+1}}{n^{s}}=\left(1-2^{1-s}\right) \zeta(s),
$$

with $\zeta(s)$ the Riemann zeta function, so the volume of $\mathscr{M}_{g, s, r}$, for odd $r$, is an elementary multiple of a value of the zeta function at an odd positive integer. For even $r,(4.93)$ shows that as regards the volume of moduli space, $r$ copies of $R P^{2}$ are equivalent to $r / 2$ Klein bottles.

\subsection{Twisted External States}

We will now use the same methods to compute the volume of the moduli space of flat connections on a punctured surface, with given monodromies around the punctures.

Let $\Sigma$ be a compact surface without boundary, and let $\Sigma^{\prime}=\Sigma-\left\{P_{1}, \ldots, P_{n}\right\}$ be the surface obtained by deleting marked points $P_{1}, \ldots, P_{n}$. Let $\Theta_{i}$ be conjugacy classes in $G$, and let $\mathscr{M}_{\{\Theta\}}$ be the moduli space of flat connections on $\Sigma^{\prime}$ with holonomy about $P_{i}$ in the conjugacy class $\Theta_{i}$. A symplectic structure on $\mathscr{M}_{\{\Theta\}}$ is defined much as in the unpunctured case. The tangent space to $\mathscr{M}_{\{\Theta\}}$ at an irreducible flat connection is $H_{c}^{1}\left(\Sigma^{\prime}, \operatorname{ad}(E)\right)\left(H_{c}^{1}\right.$ is cohomology with compact support). Given $\operatorname{ad}(E)$-valued one forms $a$ and $b$ of compact support on $\Sigma^{\prime}$, the expression

$$
\omega(a, b)=\frac{1}{4 \pi^{2}} \int_{\Sigma} \operatorname{Tr} a \wedge b
$$

defines the symplectic structure.

Suppose that the $\Theta_{i}$ are all conjugacy classes of finite order, say of order $m$. The following device then gives a short cut to understanding the integrality properties of (4.96) (which were stated in (3.15) and used in comparing to the Verlinde formula) and will also give a short cut to understanding how to set up a combinatorial computation of the torsion. Pick (noncanonically) an $m$-fold cyclic cover $\bar{\Sigma}$ of $\Sigma$, with $m$-fold branching over the points $P_{i}$ (in doing this one may enlarge the list of $P_{i}$ to include additional punctures with monodromy one). Thus, the cyclic group $F=\mathbb{Z} / m \mathbb{Z}$ acts on $\bar{\Sigma}$, and $\Sigma=\bar{\Sigma} / F$. Let $\pi: \bar{\Sigma} \rightarrow \Sigma$ be the projection. A 
$G$ bundle and connection $E$ and $A$ on $\Sigma^{\prime}=\Sigma-\left\{P_{1}, \ldots, P_{n}\right\}$ pull back to bundles and connection $\pi^{*}(E)$ and $\pi^{*}(A)$ on $\bar{\Sigma}-\left\{P_{1}, \ldots, P_{n}\right\}$. (We will usually refer to the lifts of the $P_{i}$ to $\bar{\Sigma}$ as $P_{i}$, but will call them $\bar{P}_{i}$ when more precision is needed.) If the monodromy of $A$ around each of the $P_{i}$ is of order $m$, then $\pi^{*}(E)$ and $\pi^{*}(A)$ extend naturally to a $G$ bundle and flat connection $\bar{E}$ and $\bar{A}$ on $\bar{\Sigma}$. This operation gives a natural embedding $i: \mathscr{M}_{\{\Theta\}} \rightarrow \bar{M}$, where $\overline{\mathscr{M}}$ is the moduli space of flat connections on $\bar{\Sigma} . F$ acts on $\overline{\mathscr{M}}$, and $\mathscr{M}_{\{\Theta\}}$ is a component of the fixed point set. At the tangent space level, this implies that

$$
T \mathscr{M}_{\{\Theta\}}=\left(i^{*} T \overline{\mathscr{M}}\right)^{F},
$$

where $^{F}$ denotes the $F$ invariant part. A symplectic structure on $\overline{\mathscr{M}}$ is defined by the usual formula

$$
\bar{\omega}=\frac{1}{4 \pi^{2}} \int_{\bar{\Sigma}} \operatorname{Tr} \bar{a} \wedge \bar{b},
$$

with $\bar{a}, \bar{b}$ being $\operatorname{ad}(\bar{E})$-valued one forms on $\bar{\Sigma}$. As one has

$$
\omega=\frac{1}{m} i^{*}(\bar{\omega}),
$$

the integrality of $\bar{\omega}$ implies that $m \omega$ is integral, as was claimed in (3.15).

Preliminaries About the Torsion. The main difficulty in computing the volume of $\mathscr{M}_{\{\Theta\}}$ is to obtain a technical result comparing torsions of certain complexes associated with $\mathscr{M}_{\{\Theta\}}$. We will therefore launch on a relatively long excursion with this aim.

We want to use (4.97) to construct combinatorial models for computing $T \mathscr{M}_{\{\Theta\}}$. If $X$ is any sufficiently fine covering of $\Sigma$ by polygons, then taking the inverse images of the polygons in $X$, one gets an $F$-invariant covering $X$ of $\bar{\Sigma}$ by polygons. From the cell decomposition $\bar{X}$ of $\bar{\Sigma}$, we construct in the standard way a chain complex $\bar{C}_{\bullet}$ that computes $H_{*}(\bar{\Sigma}, \operatorname{ad}(\bar{E}))$. Of course, for an irreducible flat connection, $H_{*}(\bar{\Sigma}, \operatorname{ad}(\bar{E}))$ is zero except in dimension one. Moreover, according to (4.97), the $F$-invariant part of this is $T \mathscr{M}_{\{\Theta\}}$. Hence, if $C_{\bullet}=\bar{C}_{\bullet}^{F}$ is the $F$-invariant subcomplex of $\bar{C}_{\bullet}$, then $H_{1}\left(C_{\bullet}\right)=T \mathscr{M}_{\{\Theta\}}$. Computing the torsion of $C_{\bullet}$ will give a definition of torsion for flat connections on $\Sigma^{\prime}$ with prescribed monodromy.

In carrying out this program, there are different kinds of cell decompositions $X$ to consider. Focussing on the behavior near a particular puncture $P$ (Fig. 17), we will consider two possibilities: (i) $P$ is in the interior of one of the two-cells; (ii) $P$ is one of the vertices or zero-cells.

First we take $X$ to be of type (i). $P$ is contained in a two-cell $w \cdot \bar{w}=\pi^{-1}(w)$ is a two-cell in $\bar{\Sigma}$. By contrast, if $v$ is a cell in $\Sigma$ not containing a puncture, then $\pi^{-1}(v)$ $=\bar{v}_{1} \cup \ldots \cup \bar{v}_{m}$ is a disjoint union of $m$ cells in $\bar{\Sigma}$ that are cyclically permuted by $F$. We recall that the chain complex $\bar{C}_{\bullet}$ is a direct sum of spaces $V_{\bar{x}}$ of parallel sections of $\operatorname{ad}(\bar{E})$ over $\bar{x}$. Each of these is a copy of the Lie algebra $\mathscr{G}$. For every cell $v$ in $\Sigma$ not containing a puncture, the $F$ invariant part of $V_{\bar{v}_{1}} \oplus \ldots \oplus V_{v_{m}}$ is a copy of $\mathscr{G}$ which can be identified with the space of parallel sections of $\operatorname{ad}(E)$ over $v$; we call this $V_{v}$. If there are no punctures at all, $C_{\bullet}=\bar{C}_{\bullet}^{F}$ will thus be the standard complex computing $H_{*}(\Sigma, \operatorname{ad}(E))$.

Things are more interesting if we consider $V_{\bar{w}}$, where $P \in w$. We consider a flat connection $A$ on $\Sigma$ whose monodromy around $P$ is $U . V_{\bar{w}}$ is again a copy of $\mathscr{G}$ 

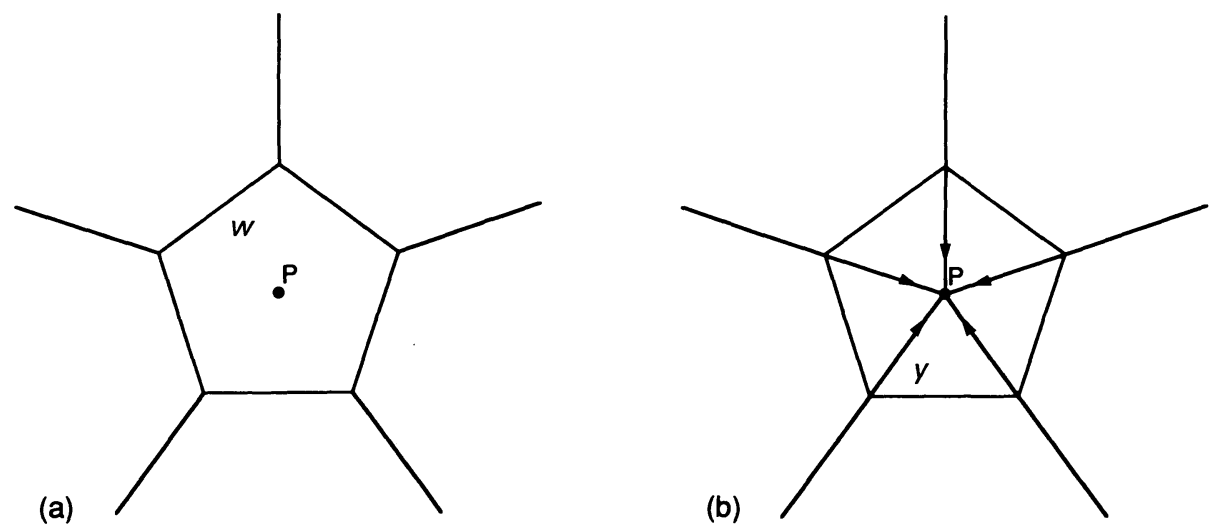

Fig. 17. Cell decompositions (i) in which a given puncture $P$ is in the interior of one of the two-cells, and (ii) in which $P$ is one of the vertices

consisting of parallel sections of $\bar{E}$ over $\bar{w}$. The generator of the cyclic group $F$ acts on $V_{\bar{w}}$ by conjugation by $U$. So $V_{w}=V_{\bar{w}}^{F}$ is just the $U$-invariant subspace of $\mathscr{G}$.

This can be stated more intrinsically: $V_{w}$ consists of parallel sections of $\operatorname{ad}(E)$ over $w-P$. In particular, if the monodromy around $P$ is trivial, then $P$ plays no role and can be reinserted in $\Sigma$. (Thus, the extra punctures with trivial monodromy that may have been introduced in constructed $\bar{\Sigma}$ can be harmlessly disposed of at this point.) However, if the holonomy around $P$ is in a generic conjugacy class, then $V_{w}$ is a copy of the Lie algebra $\mathscr{T}$ of the maximal torus $T$. Henceforth, we only consider punctures with such generic monodromies.

The boundary operator of $C_{\mathbf{0}}$, deduced by restricting the boundary operator of $\bar{C}_{\text {}}$ to the $F$-invariant part, is precisely as in Sect. 4.2. If $z$ is a $q$-cell whose boundary is a union of $q-1$-cells $y_{i}$, and $s \in V_{z}$ is a parallel section of $\operatorname{ad}(E)$ over $z($ or $z-P$ ) then $\partial s=\left.\bigoplus_{i} s\right|_{y_{i}}$.

This completes the description of the complex associated with a cell decomposition of type (i). Now we consider the case of type (ii). We consider a cell decomposition $X^{\prime}$ of $\Sigma$, with $P$ as one of the zero-cells. This lifts to a cell decomposition $\bar{X}^{\prime}$ of $\bar{\Sigma}$, with $\bar{P}$ (the lift of $P$ ) as one of the zero cells. The reasoning is then precisely as before except that now the exceptional cells are zero cells. The complex $\bar{C}_{\bullet}^{\prime}$ constructed from $\bar{X}^{\prime}$ has for its $F$ invariant part a complex $C^{\prime}$ that may be described as follows. To every cell $z$ in $X^{\prime}$ other than $P$, we associate the space $V_{z}$ of parallel sections of ad $(E)$ over $z$. To $P$, we associate the space $V_{P}$ consisting of the $U$-invariant part of the fiber of $\bar{E}$ over $\bar{P}$.

The boundary operator of $C_{*}^{\prime}$ is of course constructed by restricting the boundary operator of $\bar{C}^{\prime}$ to the $F$-invariant subspace. In describing it explicitly, there is only one subtlety. Over a neighborhood of $P, \operatorname{ad}(E)$ has a natural decomposition $\operatorname{ad}(E)=\operatorname{ad}(E)_{0} \oplus \operatorname{ad}(E)_{\perp}$, where $\operatorname{ad}(E)_{0}$ is the monodromy invariant part and $\operatorname{ad}(E)_{\perp}$ is the orthocomplement. Now, let $y$ be a one-cell in $\Sigma$ one of whose endpoints is $P$, as in Fig. 17b. In defining the boundary map $\partial: V_{y} \rightarrow V_{P}$, one first projects a parallel section $s$ of $\operatorname{ad}(E)$ onto $\operatorname{ad}(E)_{0}$ and then considers the limiting value at $P$, which is a well-defined element of $V_{P}$. Other components of the boundary map of $C_{\bullet}^{\prime}$ have the standard definition.

The standard invariance under subdivision gives a canonical isomorphism between $H_{*}\left(\bar{C}_{\bullet}\right)$ and $H_{*}\left(\bar{C}_{\bullet}^{\prime}\right)$. Taking the $F$ invariant parts, this restricts to a 


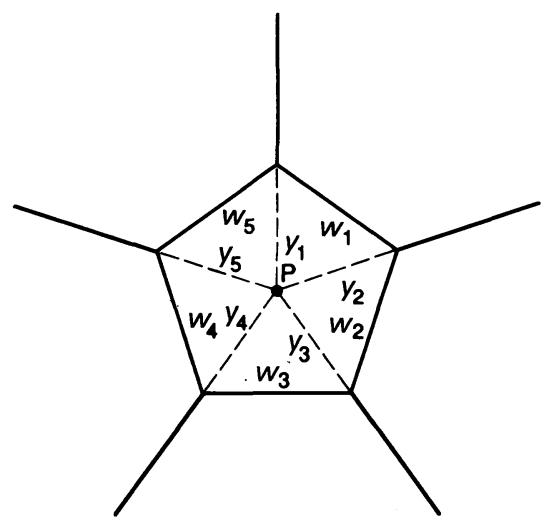

Fig. 18. Regarding $X^{\prime}$ as a subdivision of $X$. $X^{\prime}$ is created from $X$ by cutting the two-cell $w$ on new one-cells $y_{1}, \ldots, y_{n}$ to make $n$ two-cells $w_{1}, \ldots, w_{n}$

canonical isomorphism between $H_{*}\left(C_{\bullet}\right)$ and $H_{*}\left(C_{0}^{\prime}\right)$. It is instructive to see this directly. We can obtain a cell decomposition of $\Sigma$ of the type $X^{\prime}$, with $P$ as a zerocell, by subdividing a cell decomposition $X$ in which $P$ is in the interior of a two cell (Fig. 18). With the choice in the figure, in creating $X^{\prime}$, the two-cell $w$ is subdivided into $n$ two-cells $w_{1}, \ldots, w_{n}$ by cutting on one-cells $y_{1}, \ldots, y_{n}$. There is a natural injective map $i: C_{\bullet} \rightarrow C_{\bullet}^{\prime}$ of the corresponding complexes. [The definition is just like that of the map called $i$ in (4.4). In particular, if $s$ is a parallel section of ad $(E)$ over $w-P$, then $\left.i(s)=\left.\bigoplus_{i} s\right|_{w_{i} \cdot}\right]$ We let $K$. be the quotient:

$$
0 \rightarrow C_{\bullet} \stackrel{i}{\longrightarrow} C_{\bullet}^{\prime} \stackrel{f}{\longrightarrow} K_{\bullet} \rightarrow 0 .
$$

The long exact homology sequence of $(4.100)$ is the desired isomorphism of $H_{*}(C)$ and $H_{*}\left(C^{\prime}\right)$, if $H_{*}(K)=0$.

Since $i$ is an isomorphism except on the cells that are subdivided or created in going from $X$ to $X^{\prime}, K$ can be described locally, just in terms of the number $n$ of new one-cells and the monodromy $U$. In particular, $K$ has a decomposition $K=K_{0} \oplus K_{\perp}$, where $K_{0}$ is the monodromy invariant part and $K_{\perp}$ is the orthocomplement (using the metrics on the various cells). $K_{0}$ is just the tensor product with $\mathscr{T}$ of the complex defined in (4.6) and (4.7). In particular, it is acyclic and has torsion 1. $K_{\perp}$ is more interesting. The nonzero chain groups are in dimensions one and two. In dimension two, $K_{\perp 2}=\bigoplus_{i=1}^{n} V_{w_{i}}$, where $V_{w_{i}}$ consists of parallel sections of $\operatorname{ad}(E)_{\perp}$ over $w_{i}$. Likewise, in dimension one, $K_{\perp 1}=\bigoplus_{j=1}^{n} V_{y_{j}}$, where $V_{y_{j}}$ consists of parallel sections of $\operatorname{ad}(E)_{\perp}$ over $y_{j}$. For $s_{i} \in V_{w_{i}}$, the boundary operator is of course

$$
\partial s_{i}=\left.s_{i}\right|_{y_{i+1}}-\left.s_{i}\right|_{y_{i}} \in V_{y_{i+1}} \oplus V_{y_{i}} .
$$

(We identify $y_{i+n}$ with $y_{i}$.) This complex is acyclic (since in defining $K_{\perp} \bullet$ we have removed the monodromy invariants), completing the proof of isomorphism of $H_{*}(C)$ and $H_{*}\left(C^{\prime}\right)$.

Though $K_{0} \bullet$ has torsion 1 , this is not true for $K_{\perp} \bullet$. In fact, the complex (4.58) that computes the torsion of a connection on a circle is the same as the $n=1$ case of $K_{\perp}$, 
except that in (4.58) the dimensions are 0 and 1 instead of 1 and 2. The complex $K_{\perp}$, with dimensions shifted, is actually the complex that we would get to compute the torsion of a connection on a circle, provided that we had covered the circle with $n$ one-cells and $n$ zero-cells, as opposed to the choice $n=1$ that was used to derive (4.58). The dimension-shifting by an odd amount replaces the torsion by its inverse, so in contrast to (4.59), the torsion of $K_{\perp}$ is

$$
\tau\left(K_{\perp}\right)=\frac{1}{F(U)} .
$$

The volume-exactness of (4.100) thus gives us the result

$$
\tau\left(C_{\bullet}^{\prime}\right)=\tau\left(C_{\bullet}\right) \cdot \frac{1}{F(U)},
$$

if there is only one puncture with non-trivial monodromy. In general, if there are generic (regular) monodromies about several punctures $P_{1}, \ldots, P_{n}$, and $\Theta_{i}$ is the conjugacy class of the monodromy about $P_{i}$, then

$$
\tau\left(C_{\bullet}^{\prime}\right)=\tau\left(C_{\bullet}\right) \cdot \prod_{i=1}^{n} \frac{1}{F\left(\Theta_{i}\right)} .
$$

This is the technical result that we have been aiming for. The complexes $C_{\bullet}^{\prime}$ and $C_{\bullet}$ both compute $T \mathscr{M}_{\{\boldsymbol{\theta}\}}$, but their torsions differ. The rest of the discussion is relatively routine.

Comparison with the Symplectic Volume. We want to compare the volume forms on $\mathscr{M}_{\{\Theta\}}$ that can be defined using the torsion with the symplectic volume form $\omega^{r} / r$ !, with $r=\operatorname{dim} \mathscr{M}_{\{\Theta\}} / 2$. The strategy of the computation will be the same as it was in Sect. 4.2, where we considered the unpunctured case. We consider a cell division $X$ of $\Sigma$ and a dual cell division $X^{\prime}$, which determine chain complexes $C_{\bullet}$ and $C_{\bullet}^{\prime}$, in such a way that $D_{\bullet}=C_{\bullet} \oplus C_{\bullet}^{\prime}$ has a natural structure of a symplectic complex. The torsion of $D_{\bullet}$ is then "trivial" and can be related to the symplectic volume. In the unpunctured case, the formula so obtained is (4.28). We now reconsider this in the punctured case.

In the punctured case, if the complex $X$ is of type (i), with a given puncture $P$ in the interior of one of the two-cells, then to have a natural intersection pairing between $C_{\bullet}$ and $C_{0}^{\prime}$, we must take $P$ to be a vertex in $C_{0}^{\prime}$, as shown in Fig. 19. In this case, the symplectic pairing $\lambda$ on $D_{\bullet}=C_{\bullet} \oplus C_{\bullet}^{\prime}$ is defined precisely as in (4.20), and exactly as in (4.22) we get

$$
\tau\left(D_{\mathbf{0}}\right)=\left(\operatorname{Pfaff} \bar{\lambda}_{1,1}\right)^{-1} \text {. }
$$

Now, however, we have because of (4.104),

so

$$
\tau\left(D_{\bullet}\right)=\tau\left(C_{\bullet}\right) \tau\left(C_{\bullet}^{\prime}\right)=\tau\left(C_{\bullet}\right)^{2} \cdot \prod_{i=1}^{n} \frac{1}{F\left(\Theta_{i}\right)},
$$

$$
\sqrt{\tau\left(D_{\bullet}\right)}=\tau\left(C_{\bullet}\right) \cdot \prod_{i=1}^{n} \frac{1}{\sqrt{F\left(\Theta_{i}\right)}} .
$$

As $\sqrt{\tau\left(\mathrm{D}_{\mathrm{o}}\right)}$ is related to the symplectic volume by precisely the considerations of Sect. 4.2, the analog of (4.28) is

$$
\operatorname{Vol}\left(\mathscr{M}_{\{\Theta\}}\right)=\frac{1}{(2 \pi)^{\operatorname{dim} \mathscr{M}_{(\Theta)}}} \cdot \int_{\mathscr{M}_{(\Theta)}} \tau\left(C_{\bullet}\right) \cdot \prod_{i=1}^{n} \frac{1}{\sqrt{F\left(\Theta_{i}\right)}} .
$$




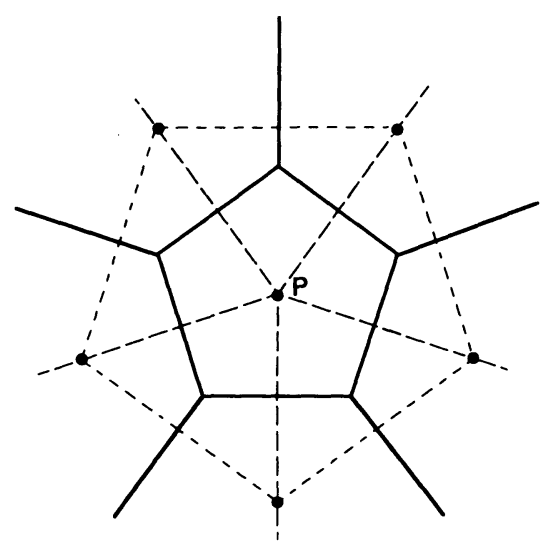

Fig. 19. A complex of type (i) (solid lines) and its dual (dotted lines), which is of type (ii)

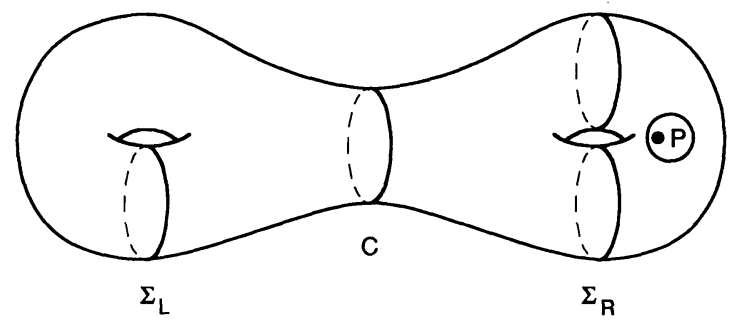

Fig. 20. A sphere of genus $g$ with $n$ punctures can be built by gluing together $2 g-2+n$ three holed spheres and $n$ once punctured discs. This is illustrated here for $g=2, n=1$

With this equation in hand, our task is elementary. An orientable surface $\Sigma^{\prime}$ of genus $g$ with $n$ punctures can be built by gluing together $2 g-2+n$ three holed spheres $W_{i}$ and $n$ once punctured discs $D_{\sigma}$ along $3 g-3+2 n$ circles $S_{\gamma}$ (Fig. 20). As we already know the torsion of the three holed sphere and the circle, we must compute the torsion of a flat connection on the once-punctured disc. Then we can repeat the standard analysis using the generalized Mayer-Vietoris formula. One could also, of course, use similar methods to compute the volume of $\mathscr{M}_{\left\{\theta_{\}}\right.}$for $\Sigma$ an unoriented surface; this involves no ideas that we have not already introduced, and will not be considered here.

The Torsion of the Punctured Disc. We now let $D$ be a disc, and let $D^{\prime}=D-\{P\}$ be a disc with one point $P$ deleted. We now want to compute the torsion of a flat connection on $D^{\prime}$ with given monodromy about $P$. We pick a conjugacy class $\Theta$ and let $\mathscr{D}_{\theta}$ be the moduli space of flat connections on $D^{\prime}$ with monodromy about $P$ conjugate to $\Theta$. Actually, any connection with this property is gauge equivalent to a standard one, so $\mathscr{D}_{\mathscr{A}}$ consists of only one point. This one point corresponds to a connection that is reducible. We will consider only the case of a generic conjugacy class for which the stabilizer is a copy of the maximal torus $T$.

In Fig. 21 we indicate a cell decomposition of $D$ which is of type (i) in our above terminology. There is a single two-cell $w$, with $P$ in its interior. The boundary of $D$ has been divided into a certain number of one-cells and an equal number of zero- 


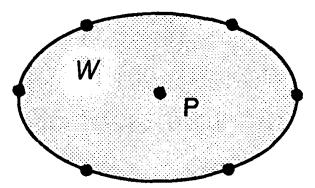

Fig. 21. The cell decomposition used to compute the torsion of a connection on the punctured disc

cells. This decomposition determines a chain complex $C_{\bullet}$ whose torsion we will compute. The homology of this complex is $H_{0}(C) \cong \mathscr{T}$ (because $\mathscr{T}$ is the Lie algebra of the stabilizer group), and $H_{1}(C)=H_{2}(C)=0$ (the vanishing of $H_{1}$ is related to the fact that any deformation of the flat connection preserving the monodromy is gauge equivalent to zero). To verify these assertions and compute the torsion, we use the decomposition $C_{\bullet}=C_{0 \bullet} \oplus C_{\perp \bullet}$, where $C_{0 \bullet}$ is the monodromy invariant part and $C_{\perp}$ is the orthocomplement. It follows from this decomposition that

$$
\tau\left(C_{\bullet}\right)=\tau\left(C_{0 \bullet}\right) \otimes \tau\left(C_{\perp \bullet}\right) .
$$

We have $C_{0 \bullet} \cong F_{\bullet} \otimes \mathscr{T}$, where $F_{\bullet}$ is the standard complex that would compute the real homology of the unpunctured disc. Hence $H_{0}(C) \cong \mathscr{T}$, and the higher homology vanishes. It is straightforward to compute that the torsion of $C_{0}$ is "trivial" in the sense that

$$
\tau\left(C_{0 \bullet}\right)=\frac{1}{v_{0}}
$$

where as usual $v_{0}$ is the measure on $\mathscr{T}$ determined by its metric. This statement can be interpreted as a reflection of the fact that the unpunctured disc is contractible to a point.

As for $C_{\perp \bullet}$, this is again precisely the complex (4.58) that computes the torsion of a connection on the circle [or rather, $C_{\perp} \bullet$ is the generalization of (4.58) to a less economical cell decomposition of the circle], so

$$
\tau\left(C_{\perp \bullet}\right)=F(\Theta) \text {. }
$$

Combining the above results, therefore, the torsion of a flat connection on the punctured disc is

$$
\tau\left(C_{\bullet}\right)=F(\Theta) \cdot v_{0}^{-1} .
$$

The Volume of $\mathscr{M}_{\{\Theta\}}$. We can now solve our problem. We consider an orientable surface $\Sigma$ with $s$ marked points $P_{1}, \ldots, P_{s}$. We pick $s$ generic (regular) conjugacy classes $\Theta_{1}, \ldots, \Theta_{s}$, and let $\mathscr{M}_{\{\Theta\}}$ be the moduli space of flat connections on $\Sigma$ with monodromy about $P_{\sigma}$ conjugate to $\Theta_{\sigma}$, for $\sigma=1, \ldots, s$. As in Fig. 20, $\Sigma$ can be made by gluing together $2 g-2+s$ three holed spheres $W_{i}$, and $s$ punctured discs $D_{\sigma}$, along $3 g-3+2 s$ circles $C_{\gamma}$. The generalized Mayer-Vietoris formula then asserts that

$$
\tau(\Sigma)=\bigotimes_{i} \tau\left(W_{i}\right) \bigotimes_{\sigma} \tau\left(D_{\sigma}\right) \bigotimes_{\gamma} \tau\left(C_{\gamma}\right)^{-1}
$$

Since we have evaluated the various factors in (4.113), $\tau(\Sigma)$ can be computed and integrated over $\mathscr{M}_{\{\Theta\}}$ using techniques that by now are familiar. One small novelty is that the factors of $\operatorname{Vol}(T)$ no longer cancel out. This is because of the $1 / v_{0}$ in (4.112) or more fundamentally because, since a flat connection on the punctured 
disc has $T$ as a group of symmetries, there is no gluing ambiguity in gluing on a punctured disc. Another small novelty is that one must remember the factors of $1 / \sqrt{F}$ in (4.108) in computing the volume of $\mathscr{M}_{\{\Theta\}}$. One gets finally

$$
\operatorname{Vol}\left(\mathscr{M}_{\{\Theta\}}\right)=\frac{\# Z(G) \cdot \operatorname{Vol}(G)^{2 g-2+s}}{(2 \pi)^{\operatorname{dim} \mathscr{M}_{\{\Theta\}}} \cdot \operatorname{Vol}(T)^{s}} \cdot \sum_{\alpha} \frac{1}{(\operatorname{dim} \alpha)^{2 g-2+s}} \cdot \prod_{\sigma=1}^{s} \chi_{\alpha}\left(\Theta_{\sigma}\right) \cdot \sqrt{F\left(\Theta_{\sigma}\right)}
$$

For instance, for $G=S U(2), \# Z(G)=2, \operatorname{dim} \mathscr{M}_{\{\Theta\}}=6 g-6+2 s, \operatorname{Vol}(G)=2^{5 / 2} \pi^{2}$, and $\operatorname{Vol}(T)=2^{3 / 2} \pi$. Also, if $\alpha$ is the $n$ dimensional representation and $\Theta_{\gamma}$ is the conjugacy class

$$
\left(\begin{array}{cc}
e^{i \pi \theta_{\gamma}} & 0 \\
0 & e^{-i \pi \theta_{\gamma}}
\end{array}\right)
$$

then $\chi_{\alpha}\left(\Theta_{\gamma}\right)=\sin \left(\pi n \theta_{\gamma}\right) / \sin \left(\pi \theta_{\gamma}\right)$ and $F\left(\Theta_{\gamma}\right)=4 \sin ^{2}\left(\pi \theta_{\gamma}\right)$. Putting the pieces together, we get

$$
\operatorname{Vol}\left(\mathscr{M}_{\{\Theta\}}\right)=2 \cdot \frac{1}{2^{g-1} \pi^{2 g-2+s}} \sum_{n=1}^{\infty} \frac{1}{n^{2 g-2+s}} \prod_{i=1}^{s} \sin \left(\pi n \theta_{i}\right),
$$

in pleasant agreement with the expression (3.18) that we obtained previously by comparing to the Verlinde formula.

\section{References}

1. Atiyah, M.F., Bott, R.: The Yang-Mills equations over Riemann surfaces. Phil. Trans. R. Soc. London A 308, 523 (1982)

2. Goldman, W.: The symplectic nature of fundamental groups of surfaces. Adv. Math. 54, 200 (1984)

3. Ray, D., Singer, I.: R-torsion and the Laplacian on Riemannian manifolds. Adv. Math. 7, 145 (1971)

4. Narasimhan, M.S., Seshadri, C.: Stable and unitary bundles on a compact Riemann surface. Ann. Math. 82, 540 (1965)

5. Verlinde, E.: Fusion rules and modular transformations in $2 \mathrm{~d}$ conformal field theory. Nucl. Phys. B 300, 360 (1988)

6. Tsuchiya, A., Ueno, K., Yamada, Y.: Conformal field theory on universal family of stable curves with gauge symmetries. Max-Planck-Institut preprint MPI/89/18

7. Segal, G.: Two dimensional conformal field theories and modular functors. In: IXth International Conference on Mathematical Physics (Swansea, July, 1988) Simon, B., Truman, A., Davies, I.M. (eds.). Bristol: Adam Hilger (1989) 22, and preprint (to appear)

8. Witten, E.: Quantum field theory and the Jones polynomial. Commun. Math. Phys. 121, 351 (1989)

9. Apostol, T.M.: Introduction to analytic number theory. Berlin, Heidelberg, New York: Springer 1984

10. Thaddeus, M.: Conformal field theory and the moduli space of stable bundles. Oxford University preprint

11. Milnor, J.: Whitehead torsion. Bull. Am. Math. Soc. 72, 358(1966)

12. Johnson, D.: A geometric form of Casson's invariant, and its connection to Reidemeister torsion. Unpublished lecture notes

13. Freed, D.: Reidemeister torsion, spectral sequences, and Breiskorn spheres. Preprint (University of Texas)

14. Fine, D.: Quantum Yang-Mills theory on the two sphere. Commun. Math. Phys. 134, 273 (1990); Quantum Yang-Mills on a Riemann surface. Commun. Math. Phys. 140, 321-338 (1991)

15. Migdal, A.: Zh. Eksp. Teor. Fiz. 69, 810 (1975) (Sov. Phys. Jetp. 42, 413) 
16. Schwarz, A.: The partition function of degenerate quadratic functional and Ray-Singer invariants. Lett. Math. Phys. 2, 247 (1978)

17. Witten, E.: Topology-changing amplitudes in $2+1$ dimensional gravity. Nucl. Phys. 323, 113 (1989)

18. Wilson, K.: Phys. Rev. D 10, 2445 (1974)

19. Brocker, T., tom Dieck, T.: Representations of compact Lie groups. Berlin, Heidelberg, New York: Springer 1985

20. Atiyah, M.F.: Geometry and physics of knots. Cambridge: Cambridge University Press 1990

21. Jimbo, M., Miwa, T., Okada, M.: Lett. Math. Phys. 14, 123 (1987); Mod. Phys. Lett. B 1, 73 (1987); Commun. Math. Phys. 116, 507 (1988)

22. Bralic, N.: Phys. Rec. D 22, 3090 (1980)

23. Kazakov, V., Kostov, I.: Nucl. Phys. B 176, 199 (1980); Phys. Lett. B 105, 453 (1981); Nucl. Phys. B 179, 283 (1981)

24. Gross, L., King, C., Sengupta, A.: Ann. Phys. 194, 65 (1989)

25. Witten, E.: Gauge theories and integrable lattice models. Nucl. Phys. B 322, 629 (1989)

26. Bott, R.: On E. Verlinde's formula in the context of stable bundles. Int. J. Mod. Phys. 6, 2847 (1991)

27. Axelrod, S.: Ph. D. thesis (Princeton University, 1991) Axelrod, S., Witten, E.: Unpublished

28. Cheeger, J.: Analytic torsion and the heat equation. Adv. Math. 28, 233 (1978)

29. Muller, W.: Analytic torsion and the $R$-torsion of Riemannian manifolds. Ann. Math. 109 (2), 259 (1979)

30. Bar-Natan, D., Witten, E.: Perturbative expansion of Chern-Simons theory with noncompact gauge group. Commun. Math. Phys. (to appear)

Communicated by A. Jaffe 
